\title{
Targeted secretion and remodelling of pectin in response to pathogen infection
}

\author{
Dissertation \\ for the award of the degree \\ ' Ph.D. Division of Mathematics and Natural Sciences ' \\ at the Georg-August-Universität Göttingen \\ within the doctoral programme \\ "Plant Responses To Eliminate Critical Threats" \\ of the Georg-August University School of Science (GAUSS)
}

Submitted by

Athanas Guzha

From Harare, Zimbabwe

Göttingen, June 2019

Department of Plant Biochemistry

Albrecht-von-Haller-Institute for Plant Sciences

Georg-August University Goettingen 


\section{Members of the Thesis Committee}

Dr. Till Ischebeck

Dept. of Plant Biochemistry, Albrecht-von-Haller Institute for Plant Sciences

Georg-August-University of Göttingen

Prof. Dr. Volker Lipka

Dept. of Plant Cell Biology, Schwann-Schleiden Centre

Georg-August-University of Göttingen

Prof. Dr. George Haughn

Dept. of Botany

University of British Columbia

\section{Members of the Examination Board}

Dr. Till Ischebeck (1st Referee)

Dept. of Plant Biochemistry, Albrecht-von-Haller Institute for Plant Sciences

Georg-August-University of Göttingen

Prof. Dr. Volker Lipka (2 ${ }^{\text {nd }}$ Referee)

Dept. of Plant Cell Biology, Schwann-Schleiden Centre

Georg-August-University of Göttingen

\section{Further members of the Examination Board}

Prof. Dr. George Haughn

Dept. of Botany

University of British Columbia

Prof. Dr. Petr Karlovsky

Dept. Molecular Phytopathology and Mycotoxin Research

Georg-August-University of Göttingen

Prof. Dr. Kai Heimel

Dept. of Molecular Microbiology and Genetics

Georg-August-University of Göttingen

PD. Dr. Marcel Wiermer

Dept. of Molecular Biology of Plant-Microbe Interactions

Georg-August-University of Göttingen

Day of the oral examination, 15.08.2019 


\section{Affidavit}

Herewith, I declare that the present PhD thesis entitled "Targeted secretion and remodelling of pectin in response to pathogen infection"

was written on my own without other sources and aids than quoted.

Göttingen,

Athanas Guzha 


\section{Table of Contents}

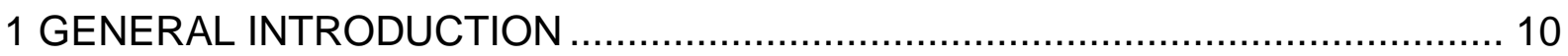

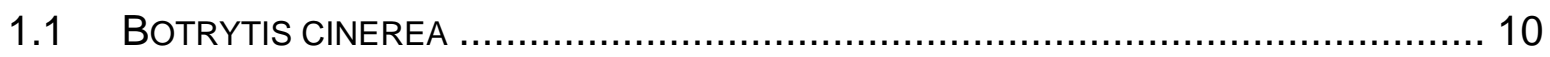

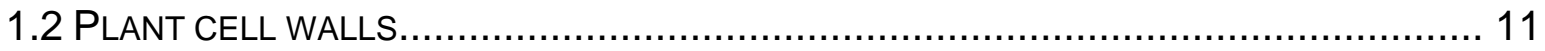

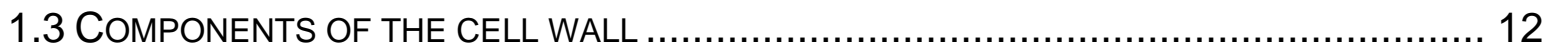

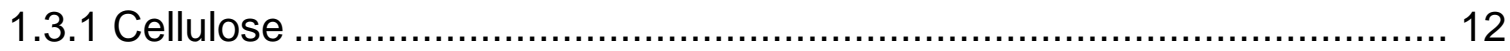

1.3.1.1 Biological activity of cellulose derived oligosaccharins ..................... 13

1.3.2 Hemicellulose .............................................................................. 13

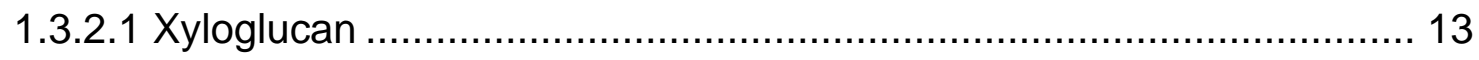

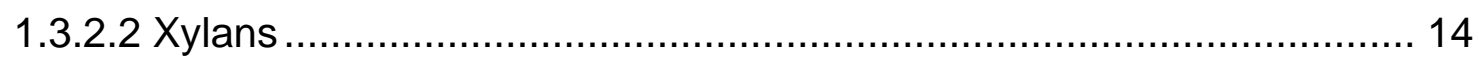

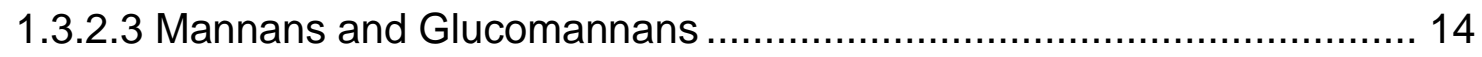

1.3.2.4 Biological activity of hemicellulose derived oligosaccharins............... 14

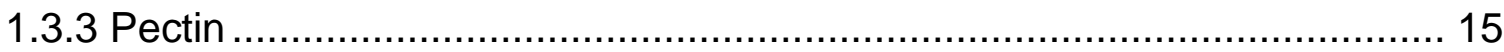

1.3.3.1 Pectin Biosynthesis ............................................................... 18

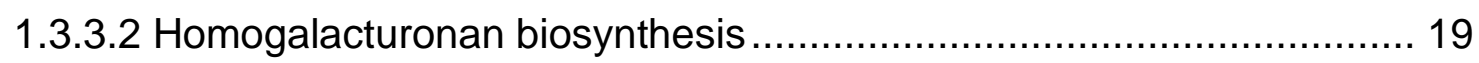

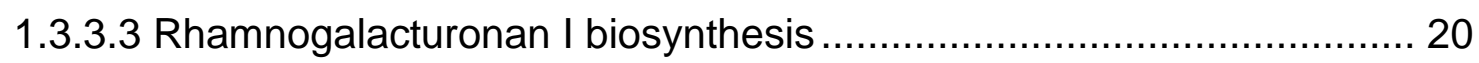

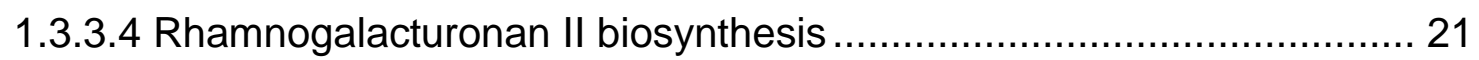

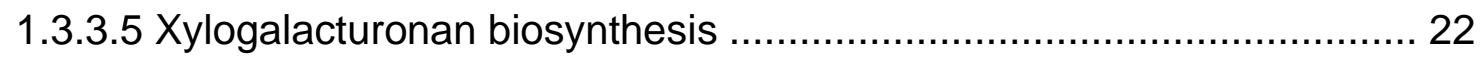

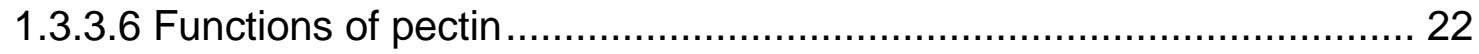

1.3.3.7 Role of pectin in plant pathogen defence ....................................... 23

1.3.3.8 Biological activity of pectin derived oligosaccharins .......................... 25

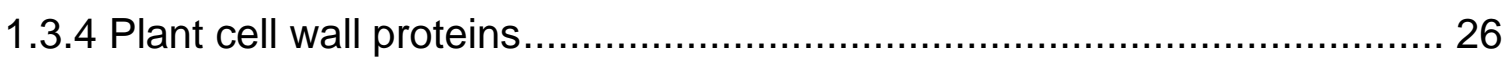

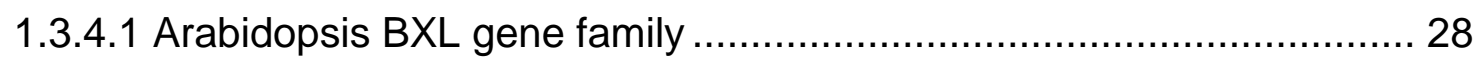

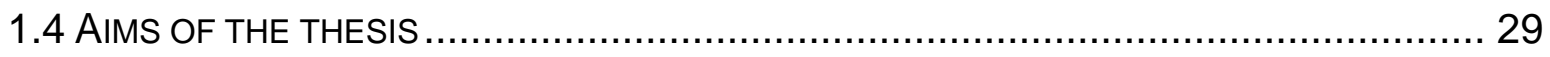

2 MANUSCRIPT A: A GC-MS-BASED METHOD OF WATER EXTRACTED PECTIN

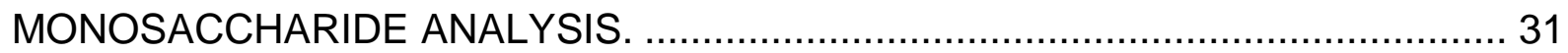

3 MANUSCRIPT B: MODIFICATION OF PECTIN RHAMNOGALACTURONAN I CONTRIBUTES TO PLANT IMMUNITY AGAINST BOTRYTIS CINEREA. ............. 53

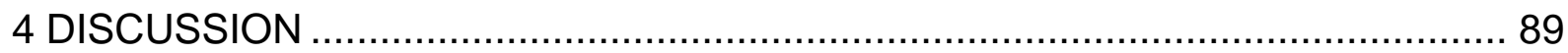

4.1 PECTIN MONOSACCHARIDE ANALYSIS USING GC-MS ..................................... 89 
4.2 BXL4 COULD ACT ON ARABINANS IN THE APOPLAST AND ITS DISRUPTION DOES NOT

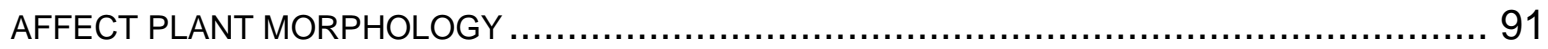

4.3 BXL4 IS A STRESS INDUCED GENE AND COULD INDUCE JA SIGNALLING .................. 94

4.4 BXL4 CONTRIBUTES TO RESISTANCE AGAINST B. CINEREA ............................... 103

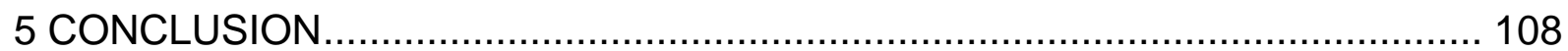

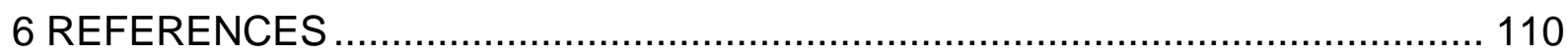

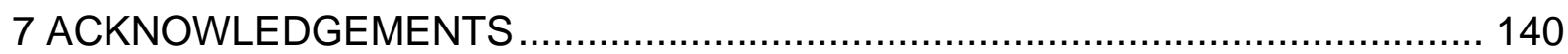




\section{Abbreviations}

ADPG2 ARABIDOPSIS POLYYGALACTURONASE2

AGP Arabinogalactan proteins

AIR Alcohol insoluble residue

AOC ALLENE OXIDE CYCLASE

AOS ALLENE OXIDASE SYNTHASE

Ara Arabinose

ARAD1 ARABINAN DEFICIENT1

AtERF014 Arabidopsis thaliana ethylene responsive factor14

BBE Berberine Bridge Enzyme

BXL1 BETAXYLOSIDASE1

BXL4 BETAXYLOSIDASE4

BXL6 BETAXYLOSIDASE6

CD Cellodextrin

CESA Cellulase synthase

COI1 CORONATINE INSENSITIVE1

CWDEs Cell wall degrading enzymes

DAMP Danger associated molecular pattern

dpa Days post anthesis

eds1 enhanced disease susceptibility 1

EXPs Expansins

GAE GLUCORONATE 4-EPIMERASE

Gal Galactose

GalA Galacturonic Acid

GAUT1 GALACTURONOSYLTRANSFERASE1

GAUT8 GALACTURONOSYLTRANSFERASE8

GC-MS Gas Chromatography-Mass Spectrometry

Glu Glucose

GluC Glucuronic Acid

GRP Glycine rich proteins

HAMPs Herbivore associated molecular patterns 
HG Homogalacturonan

HG-MT Homogalacturonan-methyltransferase

HPLC High performance liquid chromatography

HRGP Hydroxyproline rich glycoproteins

IDA INFLORESCENT DEFICIENT IN ABSCISSION

JA Jasmonic acid

JA-lle Jasmonoyl isoleucine

JAZ10 JASMONATE ZIM-DOMAIN10

LLP1 LEGUME LECTIN LIKE PROTEIN1

LOX LIPOXYGENASE

MAMP Microbe associated molecular pattern

Man Mannose

MAPK Mitogen-activated protein kinase

MBS Major beam splitter

MOX Methoxyamine hydrochloride

mpk3 mitogen-activated protein kinase3

MSTFA N-Methyl-N-(trimethylsilyl)trifluoroacetamide

MUCI70 MUCILAGE-RELATED70

OGOX1 OG OXIDASE1

OGs Oligogalacturonides

PAD3 PHYTOALEXIN DEFICIENT3

PAL PHENYLALANINE AMMONIA LYASE

PDF1.2 PLANT DEFENSIN1.2

pgm phosphoglucomutase

PLL pectate lyase like

PME Pectin methylesterase

PMEI pectin methylesterase inhibitor

pmr powdery mildew resistant

PRPs proline rich proteins

PRRs Pattern recognition receptors

PRX Peroxidase 


\begin{tabular}{ll} 
Psm & Pseudomonas syringae pv. maculicola \\
QUA1 & QUASIMODO1 \\
RG-I & Rhamnogalacturonan I \\
RG-II & Rhamnogalacturonan II \\
Rha & Rhamnose \\
ROS & Reactive Oxygen species \\
RRT & RG-I:rhamnosyltransferase \\
rwa2 & reduced wall acetylation2 \\
SA & salicylic acid \\
TBA2 & TESTA ABUNDANT2 \\
TFA & Trifluoroacetic acid \\
WAK1 & WALL ASSOCIATED KINASE1 \\
Ws & Wassilevskia \\
XcV & Xanthomonas campestris vesicatoria \\
XG & Xylogalacturonan \\
XGD1 & xylosyltransferase1 \\
XTHs & xyloglucan endo-transglycosylases/hydrolases \\
XyG & Xyloglucan \\
Xyl & Xylose \\
\hline
\end{tabular}




\section{Abstract}

Plant cell walls are a complex mix of three major classes of polysaccharides; cellulose, hemicellulose and pectin together with a minor amount of proteins, and they surround every plant cell. Besides serving as a structural component helping to maintain the shape and integrity of the plant cell. Cell walls are an interaction interface between plant cells as well as the plant and its environment and they undergo various modifications in response to developmental and environmental changes. The attack of plants by plant pathogens results in the remodelling of the pectin component in response to the pathogens. The remodelling of pectin and its implications on plantpathogen interactions are largely unknown. Here we firstly developed a robust GC-MS based method to analyse the monosaccharide profile of pectin extracted from leaves, mucilage and commercial pectin. The method is quick and easy, and both neutral sugars and galacturonic acid are quantified within the same run. We also investigate the enzymatic function of BXL4 which belongs to a seven-member betaxylosidase gene family in Arabidopsis. By expressing BXL4 in the seed coat of a bx/1 mutant, we can rescue the mucilage extrusion phenotype of $b x / 1$. Monosaccharide analysis of the complemented bx/1 and over expression lines in Col-0 show that BXL4 acts on both xylose and arabinose in the cell walls. The bx/4 mutants exhibit higher amounts of arabinose in the leaf cell walls but this does not affect the general development of the plants. Wounding and Botrytis cinerea infection of Arabidopsis show that BXL4 is a stress induced gene and its expression is JA-lle dependant. Whilst BXL4 was acting downstream of JA-lle the $b \times / 4$ mutants show a reduction in the expression of JA-Ile marker genes JAZ10 and PDF1.2 upon infection and mechanical wounding, compared to wild type. The expression of $P A D 3$ is also reduced in the $b x / 4$ mutant compared to wild type after $B$. cinerea infection. Accumulation of $\mathrm{JA}$-lle and camalexin is reduced in the $b x / 4$ mutants after infection with $B$. cinerea as compared to wild type plants. Infection of the plants with $B$. cinerea shows that the $b x / 4$ mutants have an enhanced susceptibility to the pathogen. The post secretion remodelling of pectin by BXL4 is thus an important component of plant immunity against $B$. cinerea, and this is likely due to its role in JA-lle signalling and camalexin biosynthesis. 


\section{General Introduction}

The growing human population, projected to reach 10.9 billion by 2100 (Population and development review, 2013), means an ever-increasing demand for agricultural produce from finite land resources. This calls for the development and adoption of strategies that increase the plant biomass accumulation from a unit landholding in a sustainable manner. The development of novel strategies that protect the crop against pathogen threats is fundamental to attain food security. After the cuticle, the plant cell wall acts as a second barrier that restrict the entry of pathogens. Plant cell walls are also an abundant source of renewable energy. The cell walls of multicellular land plants are a complex mix of various polysaccharides that can be used for various applications in different technological fields. Modification of plant cell walls of important crops to enhance recalcitrance against pests and pathogens is a sustainable approach to mitigate yield losses.

\subsection{Botrytis cinerea}

Plant pathogens are a ubiquitous group of organisms that contribute to crop yield losses ranging from $13 \%$ to $16 \%$ across the world every year (Vurro et al., 2010). Generally, plant pathogens are divided into three groups according to their infection strategy which are; necrotrophs, biotrophs, and hemibiotrophs (Laluk and Mengiste, 2010). Biotrophic and hemibiotrophic pathogens keep their host alive at least during some stage of their infection cycle whilst the necrotrophs kill their host tissues and proliferate in the wreckage ( Shlezinger et al., 2011; Spanu and Panstruga, 2017). Botrytis cinerea is a fungal necrotrophic pathogen with a wide host range, that is responsible for pre- and post-harvest damage in many crops (Williamson et al., 2007) causing economic losses exceeding $\$ 10$ billion across the world (Weiberg et al., 2013). The necrotrophic pathogen is able to colonise its host through the secretion of an array of cell wall degrading enzymes (Di Matteo et al., 2006). The B. cinerea infection cycle involves the attachment of conidiospores on a plant surface, followed by germination and penetration of the plant cell wall via an appressorium, aided by the secretion of cell wall degrading enzymes (Brandhoff et al., 2017). To facilitate development and spread of the hyphae, $B$. cinerea kills the host cells allowing proliferation of the hyphae in the dead tissues (Clark, 1976). The use of brute force is not the only strategy utilised by $B$. cinerea to infect its host, but it is also known to manipulate the host defence mechanism and hinder the activation of defence responses (Laluk and Mengiste, 
2010). The infection of plants by $B$. cinerea also requires the active participation of the host. Cuticle penetration together with the development of the primary lesion by $B$. cinerea results in oxidative burst at the plasma membrane and extracellular matrix near the fungal hyphae (Schouten et al., 2002). This results in disruption of the redox status close to the infection site and thereby promotes the development of the fungus (Lyon et al., 2007). B. cinerea develops sclerotia within the decomposing host debris. This serves as an important survival strategy, as sclerotia are able to withstand microbial attack, UV radiation and desiccation (Backhouse and Willetts, 1984). Because of its large host range, an array of infection strategies and ability to survive harsh conditions, B. cinerea is difficult to control. Natural plant defences against this pathogen are constitutive, involving the pre-formed barriers such as cell walls and cuticle, or inducible chemical and physical barriers (Williamson et al., 2007).

\subsection{Plant cell walls}

Plant cell walls are an important component that performs a diversity of functions such as provision of mechanical support, cell-to-cell communication, water transport, cell growth and development, and defence against biotic and abiotic factors (Cosgrove, 2005; Caffall and Mohnen, 2009). Plant cell walls usually consist of a primary cell wall found around actively growing and expanding cells, or secondary cell walls which form in specialised cells and may become fortified with lignin depositions such as occurs in vascular elements or fibre cells (Keegstra, 2010).

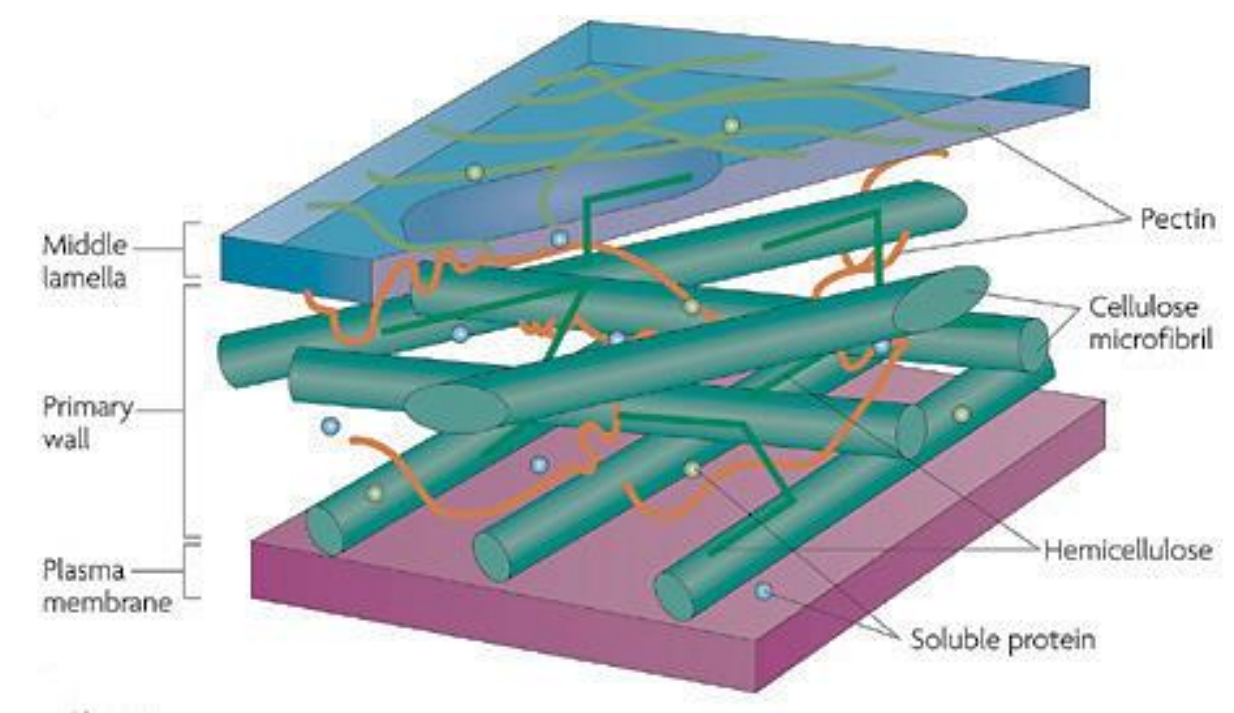

Figure 1: Model showing the major components of the plant cell walls of multicellular land plants. Cellulose microfibrils form mechanical structures that are 
cross-linked by various hemicelluloses, embedded in a pectin matrix. A small component consists of structural proteins and enzymes involved in cell wall modifications. Image adapted from Sticklen, (2008).

\subsection{Components of the cell wall}

Plant cell walls consist of a heterogeneous mixture of polysaccharides, phenols, proteins and ions (McCann and Carpita, 2008). The polysaccharide composition of cell walls of vascular plants varies from species to species, but on average, cellulose constitutes 30\%, hemicellulose 30\% and pectin 35\% (Ochoa-Villarreal et al., 2012).

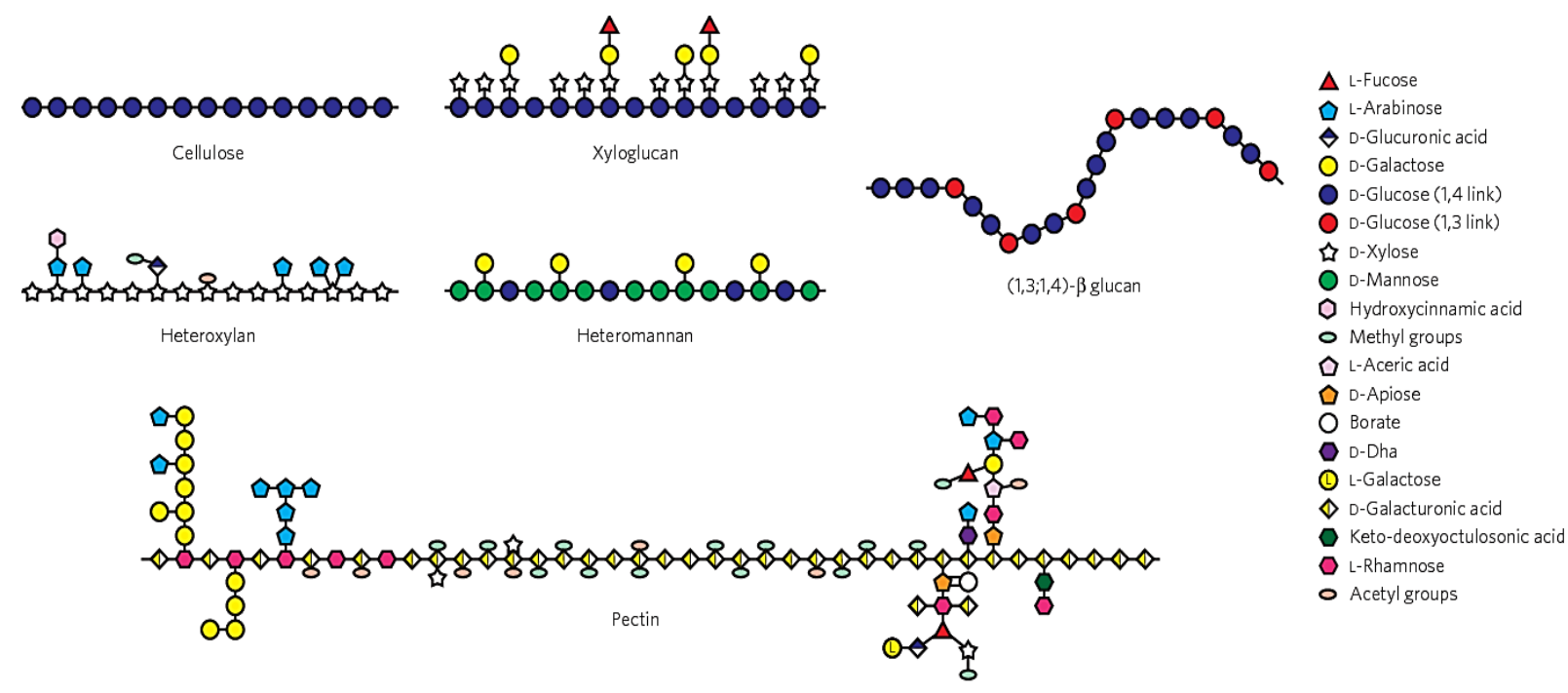

Figure 2: Cell walls are a complex mix of diverse polysaccharides linked by various glycosyl linkages. Image adapted from Burton et al., (2010).

\subsubsection{Cellulose}

Cellulose represents the main structural component of the cell walls. It consists of an unbranched polymer of $\beta$-(1,4)-D-Glucose moieties that interact together through hydrogen bonding and Van der Waals forces forming insoluble paracrystalline structures (Nishiyama et al., 2002; Nishiyama et al., 2003; Somerville, 2006). Cellulose microfibrils are built by the aggregation of cellulose chains forming insoluble structures, which are resistant to enzyme attack and are chemically stable. Cellulose is composed of two types of microfibrils, namely cellulose $1 \alpha$ and $1 \beta$ (Brown, 1996). Cellulose biosynthesis is performed by plasma membrane bound cellulase synthase (CESA) complexes that catalyse the polymerisation of glucan moieties. Ten CESA genes have so far been identified in Arabidopsis (Holland et al., 2000; Richmond, 2000). CESA 
complexes comprise of a number of subunits, which form a rosette of six CESA subunits that synthesise cellulose chains.

\subsubsection{Biological activity of cellulose derived oligosaccharins}

Hydrolysis of cell wall polysaccharides results in the generation of short chain sugars joined by glycosidic linkages that are termed oligosaccharides (Côté and Hahn, 1994). Some of these oligosaccharides can elicit a biological response in plants and they are called oligosaccharins (Darvill et al., 1992). Until recently, it was thought that only fragments generated from degradation of non-cellulosic components had biological activity. It has however been observed that cellulose derived oligasaccharins known as cellodextrins (CDs) trigger a number of plant defence mechanisms in grape plants (Aziz et al., 2007). Cellodextrins are oligomers with a linear $\beta$-(1,4)-glucose backbone and result from the hydrolysis of cellulose. PHENYLALANINE AMMONIA LYASE $(P A L)$ genes are activated upon exposure of plant cells to CD leading to the enhanced production of phytoalexins, lignin, and salicylic acid (Chaliha et al., 2018). These then activate $\beta$-1,3-glucanases and chitinases, which hydrolyse fungal cell wall $\beta-1,3-$ glucanases and chitin (Farmer et al., 1991; De Lorenzo and Ferrari, 2002). Cellodextrin applied exogenously as an elicitor has been shown to trigger ROS production, activate biosynthesis of chitinases and $\beta-1,3-g$ lucanases, and to trigger defence related genes and the accumulation of cytosolic $\mathrm{Ca}^{2+}$, providing resistance against $B$. cinerea (Aziz et al., 2007). Arabidopsis treatment with CD activates the innate immunity by triggering MAPKs and accumulating cytosolic calcium after infection with Pseudomonas syringae (Souza et al., 2017).

\subsubsection{Hemicellulose}

Hemicelluloses are low molecular weight polysaccharides consisting of a $\beta-(1 \rightarrow 4)$ linked backbone made up of glucose, xylose, or mannose with short side chains that are diverse (Scheller and Ulvskov, 2010). Hemicelluloses are important for cell wall strengthening through their interaction with cellulose microfibrils and lignin in secondary cell walls (Kurata et al., 2018).

\subsubsection{Xyloglucan}

Xyloglucans (XyG) are the most abundant hemicelluloses and have been identified in all plant species, and some mosses (Moller et al., 2007; Popper, 2008). In dicotyledonous species, the most common structure consists of a repeating heptamer 
of three glucose molecules with an $\alpha$-D-xylose substitution, followed by an unsubstituted glucose (Wilder and Albersheim, 1973).

However, there are many variations found of this polysaccharide. A $\beta$-Gal or $\alpha-I-A r a f$ side chain sometimes substitutes the xylose residues. The branched XyG is more soluble than unbranched, which may correlate with their function (Scheller and Ulvskov, 2010). Cellulose and xyloglucans exist in equal proportions in dicotyledonous plants, with XyG side chains attached to cellulose microfibrils contributing to the mechanical strength of cells walls. Arabidopsis mutants with deficiencies in the biosynthesis of XyG (xx1 $x x 2)$ are slightly smaller than the wild type with weakened hypocotyls of the seedlings, suggesting a possible role in cell wall strengthening (Cavalier et al., 2008).

\subsubsection{Xylans}

Xylans form a widely varied group of hemicelluloses, whose backbone is characterised by $\beta$ - $(1,4)$-linked xylose moieties possessing side branches of $\alpha-(1,2)$ linked glucuronic acid together with 4-O-methyl glucuronic acid. Xylans that contain many of these side chains are referred to as glucuronoxylans, and they are the most common noncellulosic component of the secondary cell walls of dicotyledonous species. If the xylan backbone is decorated with arabinan residues, it is called arabinoxylan or glucuronoarabinoxylan. This type of xylan is more common in grasses than in dicotyledonous plants ( Darvill et al., 1980; Bochicchio and Reicher, 2003).

\subsubsection{Mannans and Glucomannans}

These are $\beta$-(1 $\rightarrow 4)$-linked hemicelluloses, consisting of a mannose (mannans) or glucose and mannose in a non-repeating manner (glucomannans and galactoglucomannans). Acetylation usually occurs on the mannans and glucomannans, which act as seed storage polysaccharides (Ebringerová et al., 2005; Popper, 2008).

\subsubsection{Biological activity of hemicellulose derived oligosaccharins}

The major hemicellulose in the apoplast is xyloglucan, and the biological activity of xyloglucan-derived oligosaccharins relates to their physiological function including cell elongation and expansion. In Pisum sativum, xyloglucan oligomers could trigger accelerated cell elongation of peeled stem segments (Takeda et al., 2002), whilst in Nicotiana tabacum suspension cell cultures, they led to cell expansion and cell division 
(Kaida et al., 2010). Xyloglucans were shown to elicit the activation of MAPK and defence gene expression in Vitis vinifera and Arabidopsis triggering resistance to various pathogens including $B$. cinerea and Hyaloperonospora arabidopsidis (Claverie et al., 2018). Wheat seedlings coleoptile growth was promoted by addition of an octasaccharide derived from xyloglucan (Vargas-Rechia et al., 1998). A threecomponent oligosaccharide (FucGalXyl) of xyloglucan increased the callus tissue weight, stimulated embryogenesis, and inhibited ethylene biosynthesis in Gossypium hirsutum (Rakitin et al., 2001). Galactoglucomannan, whose backbone structure consists of glucose and mannose and side chains of galactose, also produces oligosaccharides with proven biological activity. In mung bean (Vigna radiata L.) and buckthorn (Karwinskia humboldtiana), it was proven that the root morphology was modulated by galactoglucomannan oligosaccharins (Kollárová et al., 2005, 2007).

\subsubsection{Pectin}

Pectin is a complex and abundant polysaccharide component of the primary cell walls of plants performing diverse functional roles (Voragen et al., 2009). All pectin polysaccharides contain galacturonic acid making them acidic (Caffall and Mohnen, 2009). Pectin consists of more than 17 different monosaccharides bound together by various linkages (Ridley et al., 2001; O'Neill et al., 2004). The composition of pectin varies with the plant tissues together with developmental stage of cells, tissues and organs, environment and species (Zablackis et al., 1995). There are 4 major types of pectin, namely homogalacturonan (HG), rhamnogalacturonans I (RG-I) and II (RG-II) and xylogalacturonan (XG). There is no consensus on the way the different pectins are linked together. However, it is generally thought that the different types of pectin are covalently cross-linked because their isolation requires treatment with strong chemicals (Caffall and Mohnen, 2009). Therefore models support the notion that HG, XG, RG-I and RG-II link through their backbones (Coenen et al., 2007; Ishii and Matsunaga, 2001). 


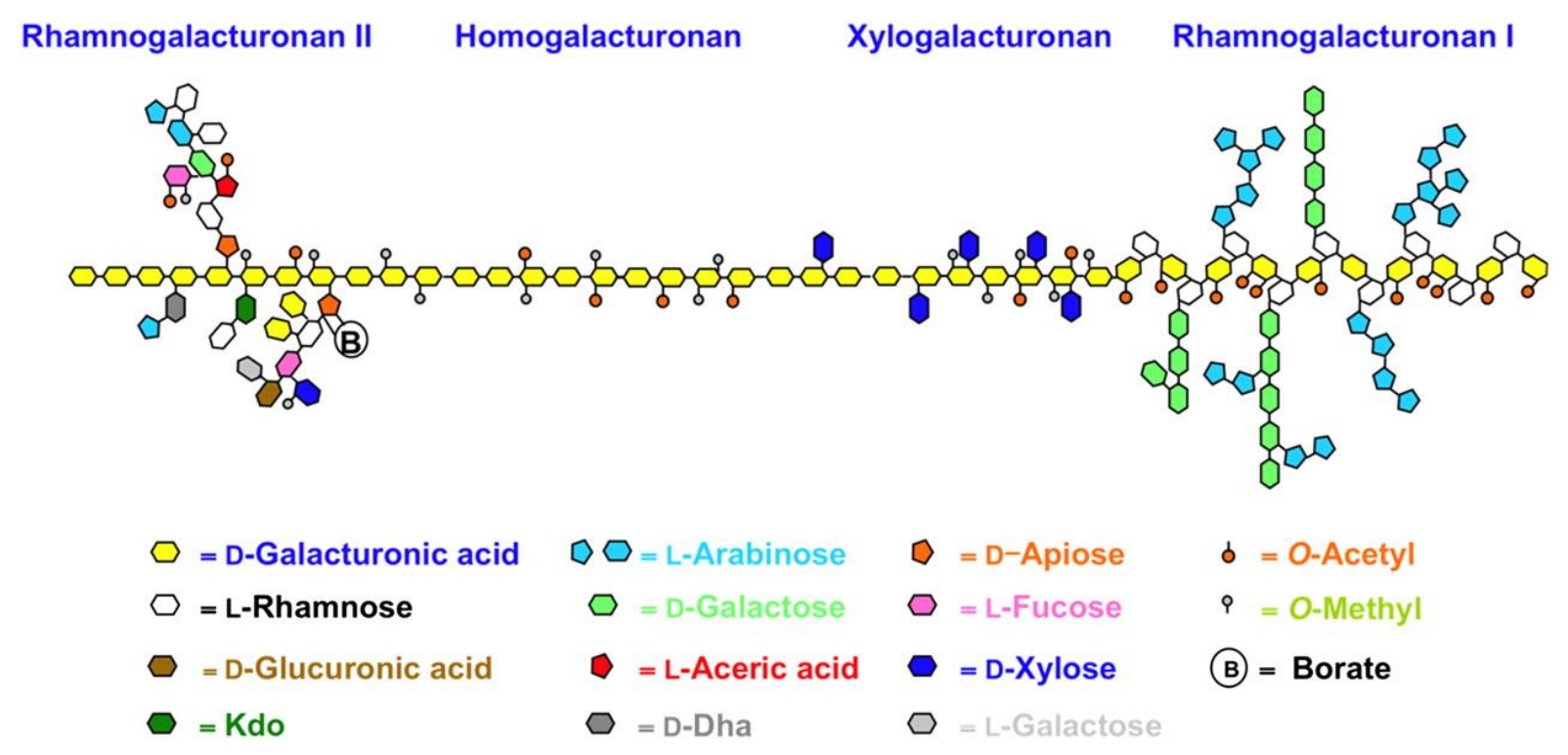

Figure 3: Pectin consists of 4 pectin polysaccharides. Pectin is characterised by the presence of a $(1 \rightarrow 4)$ a-D-galacturonic acid backbone, with the exception of rhamnogalaturonan I, which has a backbone consisting of interchanging galacturonic acid-rhamnose residues. Depending on the type of pectin, the backbone can also be methylated or acetylated and can carry side chains. Image adapted from Harholt et al., (2010).

\section{Homogalacturonan}

$\mathrm{HG}$ is the most abundant pectic polysaccharide in the leaves of Arabidopsis. HG constitutes up to $60 \%$ of all pectin polysaccharides in cell walls (O'Neill et al., 1990). $\mathrm{HG}$ is the least complex of all pectic polysaccharides consisting of an unbranched backbone of $(1 \rightarrow 4)$ a-D-galacturonic acid residues (McNeil et al., 1984; Mouille et al., 2007). Depending on the plant species, the carboxyl groups of HG are normally methylesterified at the $C-6$ position, whilst the $0-2$ and $O-3$ positions are acetyl-esterified (Ochoa-Villarreal et al., 2012). Pectin methylesterases (PMEs) are involved in the demethylation of HG (Körner et al., 2009), which is carried out in a random manner, or in a continuous block-wise manner, exposing negatively charged carboxyl groups. If at least ten galacturonic acid residues are demethylesterified, $\mathrm{Ca}^{2+}$ crosslinks this pectin forming an egg-box structure and inducing gelling of the pectin as shown in figure 4 (Raj and Ranganathan, 2012). 

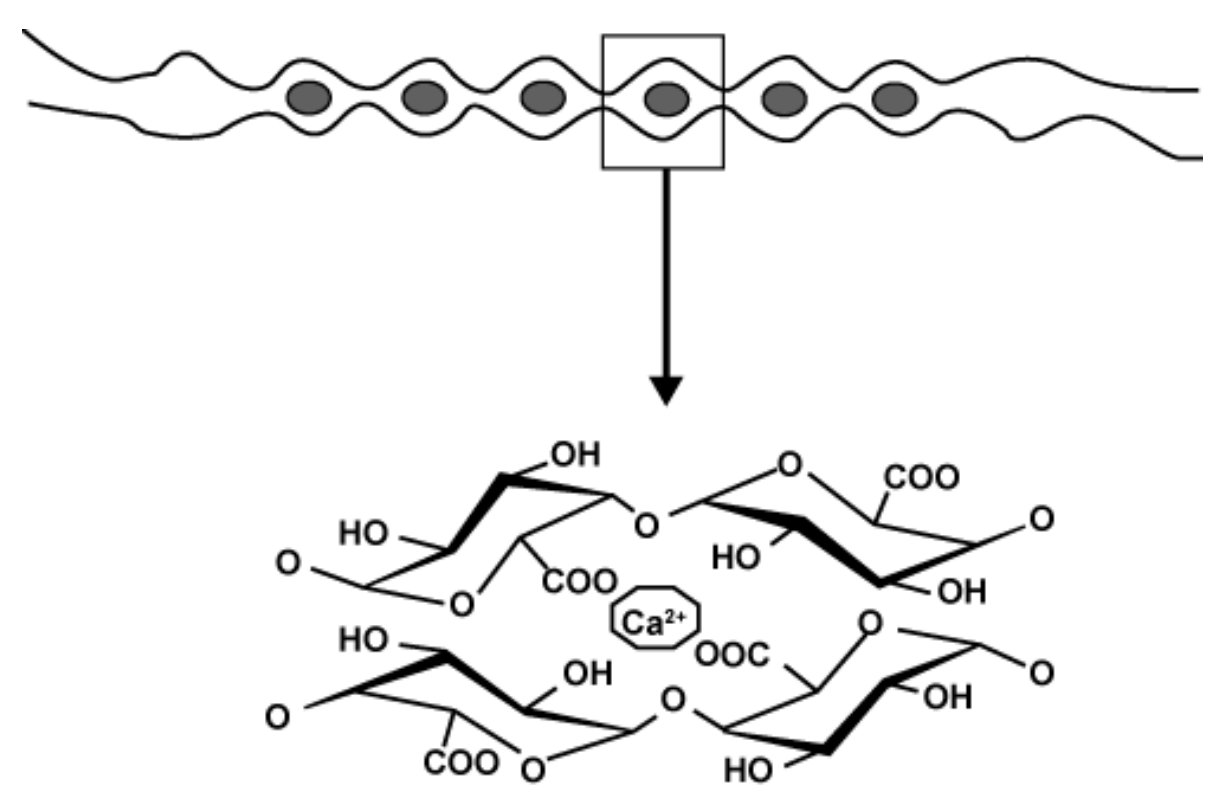

Figure 4: Demethylesterified homogalacturonan blocks are cross-linked by calcium resulting in formation of a gel. Image adopted from Raj et al., (2012).

\section{Rhamnogalacturonan I}

RG-I constitutes up to $20-35 \%$ of pectin in the plant cell wall of Arabidopsis (Mohnen, 2008). RG-I is a unique type of pectin with a backbone consisting of a repeating galacturonic acid-rhamnose disaccharide ( $\alpha-1,4-d-G a l A-\alpha-1,2-I-R h a)$ (Mohnen, 2008; Harholt et al., 2010). The rhamnose residue is usually substituted with side chains that are usually $\beta$ - $(1,4) \mathrm{D}$-galactans and/or $\alpha-(1,5)$ L-Arabinans (ØBro et al., 2004). The galacturonic residue may be acetylated on the C2 and/or C3 (Kravtchenko et al., 1992). The side chains of RG-I are heterogeneous and vary depending with plant species or tissues suggesting diverse functional roles (Mohnen, 2008).

\section{Rhamnogalacturonan II}

RG-II is the most complex pectin, and it is very conserved in the plant kingdom. RG-II is characterised by a galacturonic acid backbone with four distinct side chains namely $A, B, C$ and $D$, which contain peculiar sugars such as apiose, 3-deoxy-manno-2octulosonic acid and 3 deoxy-lyxo-2-heptulosaric acid (Ishii and Matsunaga, 2001; O'Neill et al., 2001; Ridley et al., 2001). RG-Il complexity is shown by the fact that it is made of at least 12 different sugar residues with up to 20 different glycosidic linkages involved (O'Neill et al., 2004). Despite its low abundance (0.5-8\% in dicotyledons), RG- 
II is important for proper cell wall development. RG-II is cross-linked by borate, which is important for cell wall strength (Ishii and Matsunaga, 2001; Ishii et al., 1999).

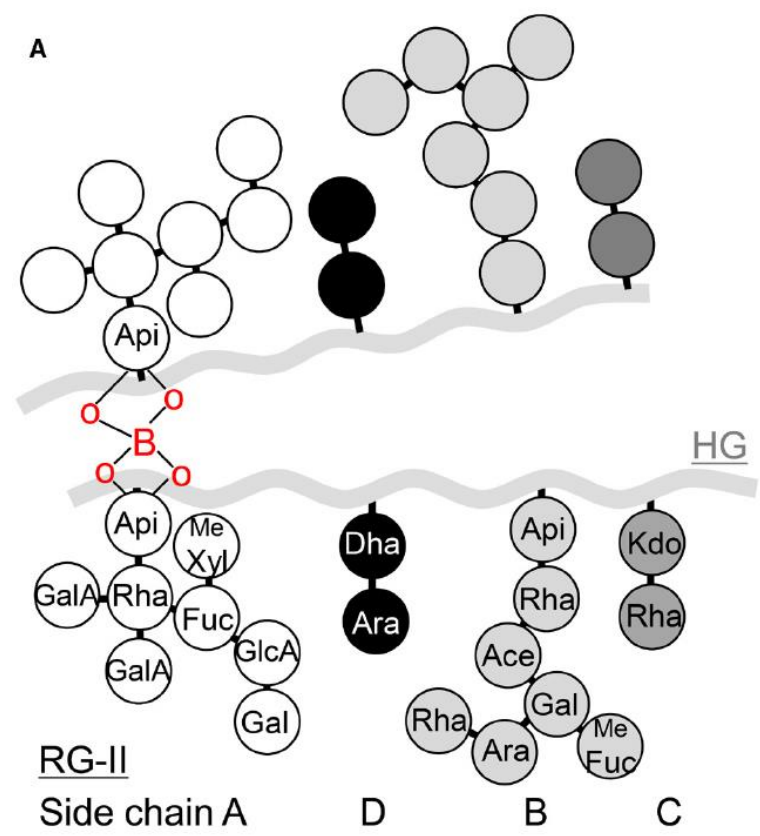

Figure 5 Model showing RG-II structure cross-linked with borate. There is the formation of a borate diester bond that covalently crosslinks the apiose residues in side chain A. Image adapted from Funakawa and Miwa, (2015).

\section{Xylogalacturonan}

The pectin xylogalacturonan consists of a backbone similar to homogalacturonan, which is a polymer of $(1 \rightarrow 4)$-linked $\alpha$-D-galacturonic acid residues. However, the backbone is substituted with $\beta$-(1-3)-xylose monomers ( Zandleven et al., 2006; Jensen et al., 2008a). Xylogalacturonan is present in most plant tissues, and accounts for $7 \%$, $6 \%$ and $2,5 \%$ of pectin extracted from young leaves, mature leaves and stems of Arabidopsis, respectively (Zandleven et al., 2007).

\subsubsection{Pectin Biosynthesis}

It is generally agreed that pectin biosynthesis occurs in the Golgi as most pectic biosynthetic activities co-fractionate with Golgi markers (Mohnen, 2008). However, the involvement of the endoplasmic reticulum during the early stages cannot be ruled out (Sterling et al., 2001; Nunan, 2003; Geshi et al., 2004). To date, few enzymes, out of the estimated 67 glycosyltransferases, acetyltransferases and methyltransferases predicted to be players in pectin biosynthesis, have been identified, and these all localize in the Golgi (Mohnen et al. 2008). In vitro enzymatic reactions have shown that glycosyltransferases involved in pectin biosynthesis utilise nucleotide sugars as 
substrate. The biosynthesis of nucleotide sugars needed to produce pectin occurs in the cytosolic side of the Golgi (Ridley et al., 2001). Transferase reactions needed to synthesis the pectin backbone together with associated side chains then takes place in the Golgi. The mechanism involved in the transport of nucleotide sugars across the Golgi membrane involves nucleotide-sugar:nucleoside monophosphate antiporters. This was confirmed in work carried out in animal systems (Capasso and Hirschberg, 1984) as well as plants (Wang et al., 1997; Neckelmann and Orellana, 1998).

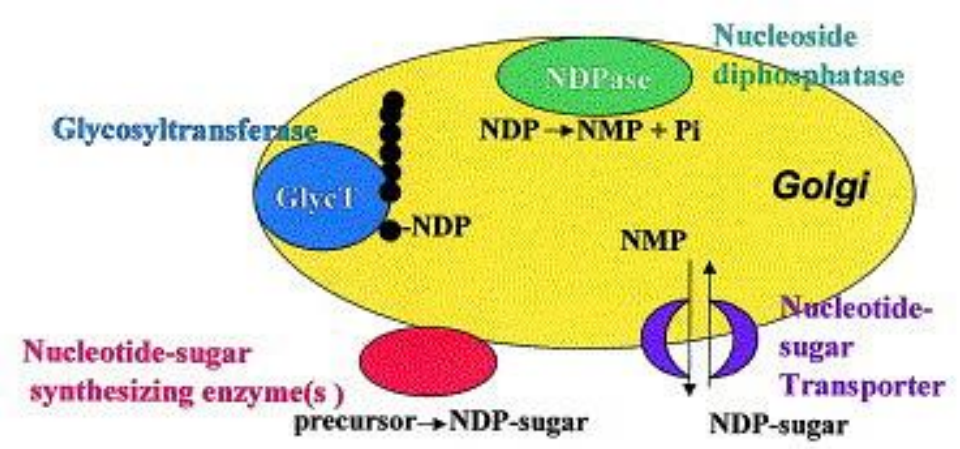

Figure 6: Model showing the biosynthetic pathway of pectin. Image taken from Ridley, et al., (2001). Nucleotide sugars are synthesised in the cytosolic side of the Golgi before nucleotide-sugar:nucleoside monophosphate antiporters transport them into the Golgi lumen. Glycosyltransferases act on the nucleotide sugars substrate by adding the glycosyl units to the growing polysaccharide chains. A nucleoside-5'diphosphatase located in the Golgi then hydrolyses the released nucleoside diphosphate to an inorganic phosphate and nucleoside monophosphate which is then made available for the nucleotide-sugar:nucleoside monophosphate antiporters (Orellana et al., 1997)

\subsubsection{Homogalacturonan biosynthesis}

There are two major enzymes catalysing the biosynthesis of homogalacturonan, namely homogalacturonan- $\alpha-1,4$ galacturonosyltransferase designated as GALACTURONOSYLTRANSFERASE (GAUT) (Sterling et al., 2006) and homogalacturonan-methyltransferase. Work by Scheller et al (1999) showed that an $\alpha-1,4-$ GalA transferase added galacturonic acid monomers to the non-reducing growing end of homogalacturonan chain in vitro (Scheller et al., 1999). GAUT1 has a predicted $\mathrm{N}$-terminal transmembrane domain with the globular domain located in the Golgi lumen. In Arabidopsis, the GAUT family contains 15 members and belongs to group 8 of CAZy GTs. Earlier work had also implicated one member of this family (QUASIMODO1 (QUA1; GAUT8)) as being a player in the production of 
homogalacturonan (Bouton et al., 2002). It is known that the methylation and polymerisation of homogalacturonan are two interdependent events during homogalacturonan biosynthesis (Mouille et al., 2007). HG-methyltransferase (HG-MT) has been identified in microsomal preparations and has been shown to methylate the pectin homogalacturonan exclusively (Mouille et al., 2007). This enzyme has been identified in mung bean (Crombie, H.J. and Reid, 1998), tobacco (Goubet and Mohnen, 1999), flax (Vannier et al., 1992), Arabidopsis (Mouille et al., 2007) and soy bean (Ishikawa et al., 2000). In the membranes of mung bean and tobacco, the methylesterification of homogalacturonan is accelerated by simultaneous addition of UDP-GalA, which is required for the synthesis of homogalacturonan (Goubet et al., 1998). The catalytic site of tobacco membrane bound HG-MT faces the Golgi lumen (Goubet and Mohnen, 1999), which is consistent with earlier observation that HG-MTs from mung bean are compartmentalised in membranes (Kauss and Swanson, 1969) and also that pea epicotyls methyltransferases localise to the Golgi (Baydoun et al., 1999). HG-MT and GalAT calatytic sites co-localise in the Golgi lumen supporting the assertion that they act one after the other in a unit during homogalacturonan biosynthesis.

The activity of a pectin $\mathrm{O}$-acetyltransferase, whose acetate donor is acetyl-CoA, has been observed in microsomal preparations obtained from suspension cell cultures of potatoes (Pauly and Scheller, 2000). In Arabidopsis, two mutants with a decrease in leaf pectin acetylation have been found and they are termed reduced wall acetylation2 (rwa2). These proteins were identified based on homology to an acetyltransferase from the fungus Cryptococcus neoformans, Cas $1 \mathrm{p}$, which is responsible for acetylation of a coat polysaccharide (Janbon et al., 2001).

\subsubsection{Rhamnogalacturonan I biosynthesis}

RG-I is a more complex pectin compared to homogalacturonan, as it possesses arabinan and galactan derived side chains (Mohnen, 2008). The increased complexity of RG-I means more enzymes are involved in its biosynthesis. Analysis of cell free homogenates of mung bean has confirmed galactosyltransferase activity, and the presence of synthesised $\beta$-1,4-galactan could confirm the presence of rhamnogalacturonan I galactosyltransferases (Panayotatos and Villemez, 1973). Membrane preparations made from hypocotyls of mung bean led to the identification of $\beta-1,4-$ galactosyltransferase activity (Brickell and Grant Reid, 1996), and also in flax 
(Peugnet et al., 2001) and potatoes (Geshi et al., 2002) activity was detected. The transfer of Gal from UDP-Gal to acceptors of RG-I has been shown and the products could only be digested by a galactosidase and not an endo- $\beta-1,4-$ galactanase (Geshi et al., 2002).

Plants possess mutases that are able to convert UDP-arabinopyranose to UDParabinofuranose, and work by Konishi et al., (2007) could prove the activity of the purified protein. Konishi et al., (2006) showed the efficient transfer of arabinofuranose onto growing oligosaccharide chains in vitro. Nevertheless, knowledge on glycosyl transferases involved in the biosynthesis of RG-I is still limited, with only a few glycosyl transferases published. Two proteins (ARABINAN DEFICIENT1 and XYLOGALACTORONAN DEFICIENT1) are membrane bound type II proteins that are localised in the Golgi (Harholt et al., 2005; Jensen et al., 2008) and involved in RG-I biosynthesis. ARABINAN DEFICIENT1 (ARAD1) is a putative arabinosyltransferase as the arad1 mutants have reduced cell wall arabinose (Harholt et al., 2005). Recently another transferase, RG-I:rhamnosyltransferase (RRT) was identified from microsomal extracts of azuki beans (Uehara et al., 2017). In vitro assays indicated that the protein could synthesis the RG-I backbone using RG-I backbone derived oligoasacharides. The transferase localised in the Golgi and endoplasmic reticulum (Uehara et al., 2017). Another glycosyl transferase MUCILAGE-RELATED70 (MUCI70) has been implicated to be involved in the biosynthesis of mucilage RG-I (Voiniciuc et al., 2018a).

\subsubsection{Rhamnogalacturonan II biosynthesis}

Not much is known about the biosynthesis of rhamnogalacturonan II, but a group of proteins (RGXT1, RGXT2, and RGXT3) belonging to Arabidopsis CAZy GT77 family was identified as having RG-II biosynthetic function (Egelund et al., 2006, 2008). The proteins are able to synthesise glycosidic linkages that only exists in RG-II and are

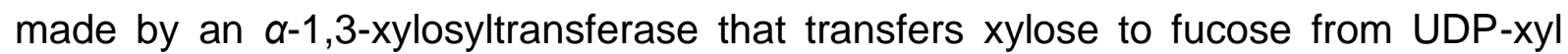
(Harholt et al., 2010). However, the activity of these proteins has only been tested in vitro using proteins isolated from insect cells that are transfected with Baculovirus (Egelund et al., 2008), and Pichia pastoris (Petersen et al., 2009).

Work by Bourlard et al., (1997) demonstrateded that a pectin methyltransferase extracted from flax cell suspension cultures can use RG-Il as an acceptor in vitro. However, the exact position where this methylation occurs is yet to be identified. Given 
the complexity of RG-I, many glycosyl transferases must be required for its biosynthesis

\subsubsection{Xylogalacturonan biosynthesis}

A xylogalacturonan xylosyltransferase (XGD1) has been characterised and is found in CAZy GT47 family. Monosaccharide and linkage analysis of mutants deficient in the enzyme have a reduction in xylogalacturonan content (Jensen et al., 2008). Microsomal preparations used in in vitro assays and transient expression assays in Nicotiana benthamiana indicated that xylose was transferred from UDP-Xyl to GalUA oligosaccharides confirming the xylosyltransferase activity of XGD1 (Jensen et al., 2008).

\subsubsection{Functions of pectin}

Pectin performs diverse functional roles during plant development. The different structural domains present in the pectin heteropolysaccharide give various physical properties attributed to pectin. Pectin can influence the way cellulose microfibrils are deposited, which in turn influences cell mechanical properties (Chanliaud and Gidley, 1999). Pectin is known to influence the ion homeostasis and $\mathrm{pH}$ of the cell wall matrix through its ability to bind calcium and boron. The binding of calcium to demethylesterified homogalacturonan forma a gel affecting the porosity and mechanical properties of cell walls (Willats et al., 2001). Pectin present in the extracellular matrix is important for cell-to-cell adhesion, and also the controlled degradation of bonds present in this pectin is a requisite for the formation of intercellular spaces present in parenchymatous tissues (Knox, 1992).

The pectin network is an important player in plant developmental processes such as fruit ripening, pod dehiscence and fruit and leaf and fruit abscission (Wen et al., 1999; Roberts, 2000). Pectin acts as a storage polymer and is important for the germination of seeds of some plant species. The arabinan and galactan side chains of RG I, because of their easy degradation, are the first polymers mobilised during germination (Tang et al., 1999). In Arabidopsis, the removal of Arabinans prior to germination negatively influences the germination process (Gomez et al., 2009). The active opening and shutting of the stomata in Arabidopsis is a function of the arabinan content present in pectin. It was shown that lack of arabinans in guard cells affected their ability to open or close (Jones et al., 2003, 2005; Merced and Renzaglia, 2019). The regulation of pollen tube tip growth is dependent on pectin and chemical nature of the 
pectin. It is known that the growing tip of pollen tubes is exclusively made out of pectin, which is strong enough to prevent bursting and at the same time flexible enough to promote elongation for successful fertilisation to take place. Altering the nature or deposition of this pectin affects pollen tube growth with negative effects on pollination (Bosch, 2005; Ischebeck et al., 2008). Homogalacturonan degradation generates oligalactoronides with biological activity influencing the expansion of cells and the triggering of plant defences (Dumville and Fry, 2000; Kohorn and Kohorn, 2012)

\subsubsection{Role of pectin in plant pathogen defence}

The importance of pectin in plant immunity is shown by the wide array of pectin hydrolysing proteins secreted by pathogens during their infection cycle. Silencing of some these enzymes renders the pathogen unable to infect the plant (Bellincampi et al., 2014; Lionetti et al., 2012). Bethke et al., (2016) indicated that reduction in pectin biosynthesis increase the susceptibility of Arabidopsis to various pathogens. Mutations in glucoronate 4-epimerase result in Arabidopsis plants with reduced cell wall pectin, because the protein is required for the biosynthesis of pectin building block UDP-Dgalacturonic acid from its precursor UDP-D-glucuronic acid. Knockout mutants have a compromised basal resistance to specific $B$. cinerea isolates and to $P$. syringae. An Arabidopsis nuclear-localised transcription factor AtERF014 was found to play a role in pectin biosynthesis, as it activates the genes involved in this process (Nakano et al., 2012). Arabidopsis AtERF014-RNAi knockdown lines have a reduction in cell wall pectin and were more susceptible to $P$. syringae, but were more resistant to $B$. cinerea (Zhang et al., 2016).

An Arabidopsis starch deficient mutant, phosphoglucomutase (pgm), that has reduced levels of cell wall pectin was shown to be susceptible to the biotrophic pathogen Colletotrichum higginsianum (Engelsdorf et al., 2017). The Arabidopsis mutant mur8-1 also exhibited an increased susceptibility to $C$. higginsianum (Engelsdorf et al., 2017). MUR8 codes for a protein involved in rhamnose biosynthesis (Mertz et al., 2012), and the knockout mutant mur8 has reduced rhamnose and RG-I content in the cell walls. However, the effects of pectin on plant defences are not always straight forward, as complex resistance phenotypes can result from changes in pectin content. For example the powdery mildew resistant 5 (pmr5), pmr6 double mutant, while being less susceptible to powdery mildew, exhibits a decreased resistance to P.syringae and Hyaperonospora arabidopsidis compared to wild type plants ( Vogel, 2002; Vogel et al., 2004). PMR6 is a putative pectate lyase, thus explaining the increased pectin 
abundance in the mutant plant cell walls, whilst PMR5 codes for a protein of a still unknown function (Maeda et al., 2009).

Changes to pectin that occur in response to interactions with pathogens are not limited only to the amount of pectin in the cell walls but also to the post-synthesis modifications. It is known that pectin upon deposition in the apoplast undergoes various modifications such as methylation, acetylation (Atmodjo et al., 2013), or trimming of galactan or arabinan side chains in RG-I (Arsovski et al., 2009; Dean et al., 2007). Multiple lines of evidence indicate that such changes to pectin composition influence plant-pathogen interactions. Homogalacturonan methylesterification is controlled by the action of pectin methylesterases, which are in turn regulated by pectin methylesterase inhibitors (PMEls) (Lionetti et al., 2012). AtPMEl10, AtPMEl11 and AtPMEl12 are pectin methylesterase inhibitors that regulate the methylesterification of pectin during infection with $B$. cinerea (Lionetti et al., 2017). Some AtPMEl overexpression lines exhibited an increased susceptibility to viral infections highlighting the importance of pectin methylesterification to plant pathogen interaction (Lionetti et al., 2014). It is thought that AtPMEl10 and AtPMEl11 are part of a plant defence system activated by the phytohormones jasmonate or ethylene (Overmyer et al., 2003) and help in resistance against $B$. cinerea in Arabidopsis (Lionetti et al., 2017). Wieczorek et al., (2014) presented evidence that pectate lyase like (PLL) genes together regulate the complexity of pectin including the degree of pectin methylesterification. In Arabidopsis, the infection with some cyst nematodes is reduced if the activity of PLL is abrogated, as the development and maintenance of the syncytia is impaired (Wieczorek et al., 2014).

Arabidopsis mutant plants with reduced wall acetylation have been identified (rwa2). The reduction in acetylation occurs on both pectin and non-pectin polysaccharides (Manabe et al., 2011). The rwa2 Arabidopsis plants show a reduced susceptibility to B. cinerea (Manabe et al., 2011; Pogorelko et al., 2013). Plants produce endogenous polygalacturonases which depolymerise pectic polysaccharides during different developmental processes such as cell expansion, leaf and flower abscission and pollen tube development (D'Ovidio et al., 2004; Hadfield and Bennett, 1998). Wang et al., (2017) could show that $P$. syringae is able to trigger the induction of IDA-like 6 (IDL6) in Arabidopsis, which then induces the expression of an Arabidopsis polygalacturonase (ADPG2). The ADPG2 overexpression lines and the adpg2 knockdown mutants had an increased resistance and susceptibility to $P$. syringae 
respectively. The activity of polygalacturonases is inhibited by polygalacturonase inhibitor proteins (PGIPs), and as a defence response to phytopathogen polygalacturonase, plant produce PGIPs. Different plant species that overexpress PGIPs have been shown to have enhanced resistance to bacteria and necrotrophic fungi (Agüero et al., 2005; Ferrari et al., 2012).

\subsubsection{Biological activity of pectin derived oligosaccharins}

Pectin depolymerisation results in the generation of fragments containing various sugar residues including galacturonic acid, rhamnose, arabinose, xylose, mannose and galactose (Ridley et al., 2001). Oligogalacturonides (OGs) are derived from the degradation of homogalacturonan and thereby form linear galacturonic acid oligomers, which may or may not be methylesterified or acetylated. OGs produced from homogalacturonan degradation by pathogens act as danger associated molecular patterns (DAMPs) that activate various plant defence responses (Boller and Felix, 2009; Tör et al., 2009; Ranf et al., 2011). Activated defence responses include callose deposition, induction of ROS species (Galletti et al., 2008), production of chitinases and glucanases ( Davis and Hahlbrock, 1987; Broekaert and Peumans, 1988), and the accumulation of phytoalexins ( Davis et al., 1986; Chassot et al., 2008; Selim et al., 2017).

The plant defence responses elicited by OGs have led to the development of the theory that plants developed a pectin integrity monitoring system (PIMS), and that OGs are key players in this system that monitors pectin perturbation either from pathogens or during normal developmental processes (De Lorenzo et al., 2011). However, the degree of polymerisation is important for OGs to elicit biological responses. Only OGs with a degree of polymerisation of 10-15 elicit biological responses (Côté and Hahn, 1994). PGIPs which retard the activity of polygalacturonases are thus an important player in plant defences, as they not only impede pectin hydrolysis but also promote the production of OGs that elicit biological responses (De Lorenzo et al., 994, 2001). However, there have been OGs with a degree of polymerisation of 2-6 that have been shown to elicit biological responses, though they suppress plant defence responses in wheat (Moerschbacher et al., 1999). OGs are known to trigger oxidative bursts in Arabidopsis resulting in the deposition of callose in the apoplast (Zhang et al., 2007; 
Galletti et al., 2008). Arabidopsis treated with OGs exhibits reduced susceptibility to phytopathogens including B. cinerea (Ferrari et al., 2007; Rasul et al., 2012).

OGs come with various degrees of methylesterification and acetylation, and it has been proven that the formation of haustoria of Blumeria graminis is impaired by acetylated OGs (Pelloux et al., 2007). Work by Randoux et al., (2010) showed that acetylated and unacetylated $O G s$ triggered hydrogen peroxide accumulation at fungal penetration site. Osorio et al., (2008) also proved that transgenic strawberries (Fragaria vesca L.) that produced demethylesterified OGs were more resistant to $B$. cinerea, thus demonstrating the effect of methylesterification on the biological activity of OGs. Biological responses elicited by pectin oligosaccharides are not only confined to plant defences, but can trigger various developmental and physiological processes. OGs are antagonistic to auxins and suppress the expression of some auxin-induced genes upon perception of auxins (Mauro et al., 2002). Consequently, coleoptile growth in maize (Zea mays L.) seedlings was reduced, and lateral rooting induced by OGs resulted in modified root structure (Hernández-Mata et al., 2010).

\subsubsection{Plant cell wall proteins}

Plant cell wall proteins constitute a minor component of cell wall, approximately $5-10 \%$ (Fry, 2004), but they are a critical player in cell wall development and responses to environment (Fry, 2004; Passardi et al., 2004). Cell wall proteins can be classified into two broad groups: structural proteins and cell wall modifying proteins.

Structural proteins vary in their abundance according to growth conditions, plant tissues and plant species. Classification of structural proteins is based on their predominant amino acids with the most common being glycine rich proteins (GRPs), hydroxyproline rich glycoproteins (HRGPs), arabinogalactan proteins (AGPs) and proline rich proteins (PRPs) (Albenne et al., 2013). Arabinogalactan proteins have been implicated to also play a role in cell signalling (Seifert and Blaukopf, 2010).

Other cell wall proteins do not have a structural role, but are capable of modifying cell wall polysaccharides. The most widely studied cell wall modifying proteins are the expansins (EXPs), which, however, have no enzymatic function that has clearly been characterised. EXPs are thought to modify interactions existing between cellulose and xyloglucans in a manner that is pH-dependent (Marowa et al., 2016). The pHdependent regulation of EXPs forms the basis of the acid-growth hypothesis, which states that cell walls expand in acidic conditions (Cosgrove, 2005). EXPs promote cell 
wall relaxation, which in turn allows for cell expansion and gives other cell wall modifying proteins access to cell wall polymers. EXPs also enhance the activity of many other cell wall-modifying proteins such as xyloglucan endotransglycosylases/hydrolases (XTHs) that are able to cleave and re-ligate xyloglucans (Eklof and Brumer, 2010).

Other cell wall proteins do not actively act on the cell polysaccharides, but their activity indirectly modifies the cell walls. Arabidopsis class III peroxidases localised in the apoplast have been identified including PRX33 and PRX34, which act as sources of apoplastic oxidative bursts in plants infected with avirulent $P$. syringae strains (Bindschedler et al., 2006). O'Brien et al., (2012) could show that Arabidopsis cell suspensions, when treated with MAMPs, produced $\mathrm{H}_{2} \mathrm{O}_{2}$, of which $50 \%$ was attributed to $P R X 33$ and PRX34. It was also shown that PRX33 and PRX34 were important for resistance against various pathogens including $B$. cinerea, Golovinomyces cichoracearum and P. syringae (Bindschedler et al., 2006). Another group of cell walllocalised proteins contributing to ROS bursts are amine oxidases and polyamine oxidases, which catabolise polyamines such as spermidine resulting in $\mathrm{H}_{2} \mathrm{O}_{2}$ production, leading to programmed cell death or triggering of stress tolerance signals (Petrov et al., 2015). Infiltration of Arabidopsis leaves with polyamines results in oxidative bursts that trigger programmed cell death (Yoda et al., 2009). Table 1 gives a summary of some major cell wall proteins identified in Arabidopsis. Many proteins modifying pectin have also been characterised for instance HG is cleaved by pectate lyases, which is important for re-arrangement of the pectin matrix (Domingo et al., 2002; Marin-Rodriguez, 2002). HG also undergoes modifications by pectin methyesterases, acetylases and polygalacturonases which have already been highlighted (Micheli, 2001; Babu and Bayer, 2014; Philippe et al., 2017). In Arabidopsis, an enzyme that oxidises OGs named OG OXIDASE1 (OGOX1) was identified (Benedetti et al., 2018). The cell wall-localised protein belongs to a large gene family of putative FAD-binding proteins named Berberine Bridge Enzyme like (BBE-like). The oxidation of OGs was found to be important for the deactivation of biologically active OGs produced after pathogen infection (Benedetti et al., 2018).

The pectin RG-I undergoes various modifications including degradation of the backbone by a rhamnogalacturonan lyase during fruit ripening (Guillermo BerumenVarela and Martín-Ernesto Tiznado-Hernández, 2018). Galactosidases and 
arabinosidases are also thought to act on pectin RG-I. Their activity is important for production of mucilage with correct hydration properties in Arabidopsis seed coat epidermal cells (Dean et al., 2007; Arsovski et al., 2009).

Table 1 Classification of Arabidopsis cell wall proteins according to predicted functional domains. Proteins are classified according to their functional domains determined using Pfam, InterPro and PROSITE bioinformatics program (Albenne, et al., 2013).

\begin{tabular}{|l|l|}
\hline Functional class & $\%$ of identified proteins \\
\hline Proteins acting on polysaccharides & 25.7 \\
\hline $\begin{array}{l}\text { Glycoside hydrolase } \\
\text { Esterases/lyases } \\
\text { Expansins }\end{array}$ & \\
\hline $\begin{array}{l}\text { Oxido-reductases } \\
\text { Peroxidases }\end{array}$ & 14.6 \\
\hline $\begin{array}{l}\text { Berberine Bridge enzymes } \\
\text { Structural proteins }\end{array}$ & \\
\hline Proteins involved in signalling & 1.6 \\
\hline Proteases & 6.6 \\
\hline Proteins with interacting domains & 11.2 \\
\hline $\begin{array}{l}\text { Lectin domain } \\
\text { LRR domain } \\
\text { Enzyme inhibitors }\end{array}$ & 11 \\
\hline Proteins related to lipid metabolism & \\
\hline Miscellaneous & \\
\hline Unknown function & 5.8 \\
\hline & 11 \\
\hline
\end{tabular}

\subsubsection{Arabidopsis BXL gene family}

The analysis of Arabidopsis crude protein extracts from stem tissues led to the identification of two proteins exhibiting betaxylosidase activity namely; BXL1 and BXL4 (Minic et al., 2004). Previous work had also identified BXL1 in a screen for proteins with a possible function in secondary cell wall thickening (Goujon et al., 2003). Additional work confirmed that BXL1 belonged to a small gene family of xylosidases or beta-xylosidase due to the presence of a glycosyl hydrolase domain and their similarity to bacterial and fungal beta-xylosidases (Goujon et al., 2003). The other members of this gene family are BXL2, 3, 4, 5, 6 and 7 (figure 7). BXL1 is the only fully characterised member of this gene family and according to Arsovski et al., (2009), it is a bifunctional $\beta$-d-xylosidase/a-l-arabinofuranosidase. Mutations in $B X L 1$ result in plants with seed mucilage that cannot extrude due to an increase in Ara side chains of RG-I (Arsovski et al., 2009). $B X L 1$ expression is confined to tissues undergoing thickening of the 
secondary cell walls and the knockout mutants have a reduced beta-xylosidase activity (Goujon et al., 2003). BXL2, 4 and 5 all have a predicted extracellular localisation, and are therefore possibly involved in cell wall remodelling as well (Goujon et al., 2003).

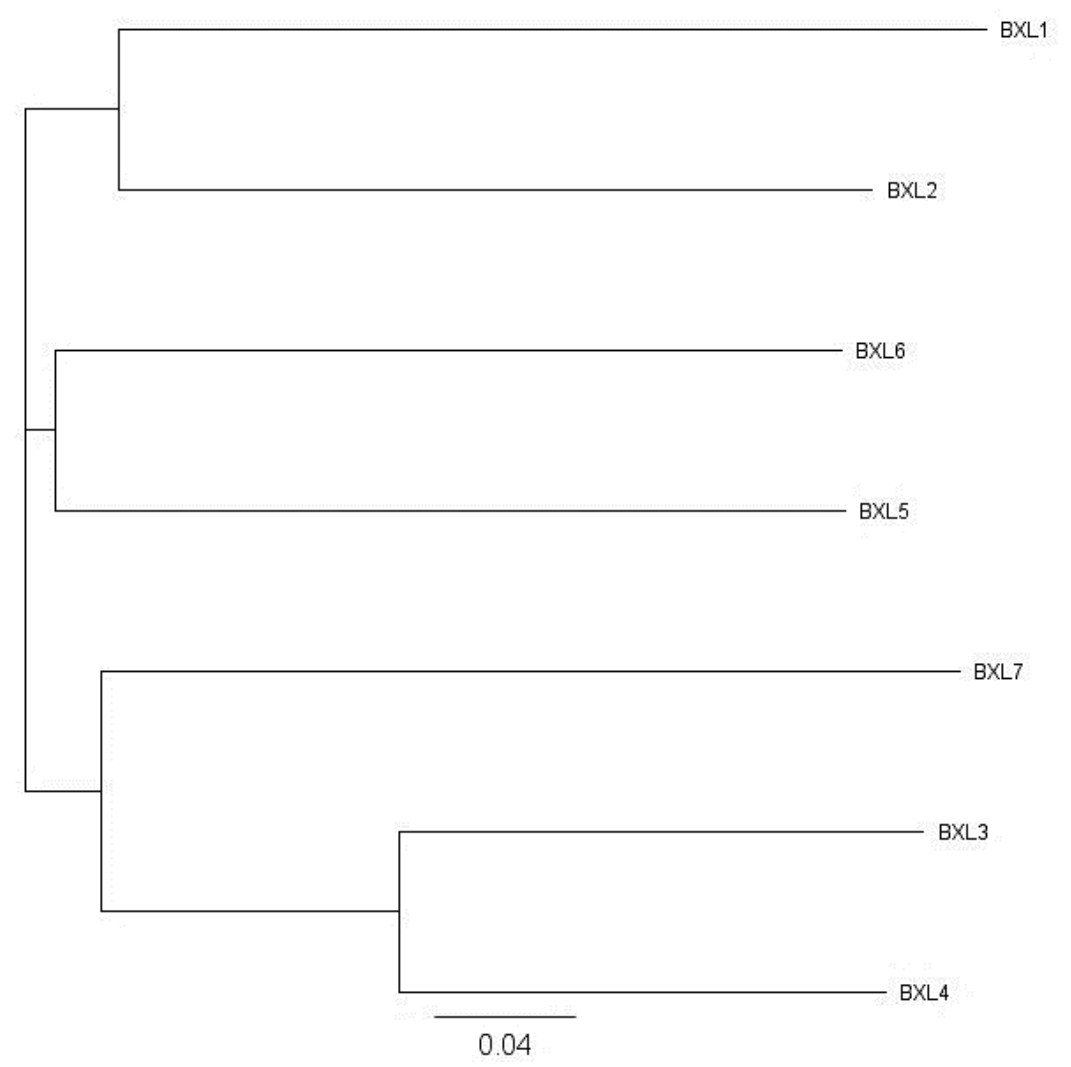

Figure 7: Phylogenetic tree of betaxylosidases (BXLs) from Arabidopsis thaliana. Image generated with Geneious version 8.1 created by Biomatters (http://www.geneious.com) using Jukes-Cantor genetic distance model and Neighborjoining tree build method

\subsection{Aims of the thesis}

As already highlighted Arabidopsis BXL1 is a known bifunctional $\beta$-d-Xylosidase/ $\alpha-I-$ Arabinofuranosidase belonging to a seven member gene family in Arabidopsis and acts on xylan and arabinan chains present in cell walls (Goujon et al., 2003; Minic et al., 2004; Arsovski et al., 2009). The enzyme activity of the other members of this BXL family are yet to be ascertained, presenting the first objective of this thesis. This work aims to determine the functional activity of BXL4 with regards to cell wall modifications through expression in Arabidopsis seed coat epidermal cells of the bx/1 knockout mutant and analysis of cell walls of bx/4 knockout mutants. 
The importance of plant cell modifications in plant pathogen defence responses in Arabidopsis has already been indicated in this paper (Bacete et al., 2018; Novaković et al., 2018). Transcriptome databases such as eFP browser and Genvestigator show that $B X L 4$ is upregulated upon infection of Arabidopsis by various phytopathogens (Breitenbach et al., 2014) thus, might be involved in cell wall remodelling to mitigate pathogen development in Arabidopsis. The second aim was to determine the cell wall modifications induced by BXL4 and its effect on plant immunity. $b x / 4$ mutants were assayed for their susceptibility/resistance to $B$. cinerea.

The third aim of the thesis is to develop a gas chromatography-mass spectrometry (GC-MS) based protocol to analyse monosaccharide compositions of pectin extracted from various tissues including mucilage and leaves. The protocol developed should reliably determine monosaccharide compositions of both neutral sugars and galacturonic acid of extracted pectin and is needed to pursue the first aim. 


\section{Manuscript A: A GC-MS-based method of water extracted pectin monosaccharide analysis.}

This manuscript is due for submission to Plant Methods journal.

Individual contribution:

Athanas Guzha performed all the experiments together with Patricia Scholz. He also did the data analysis and wrote the manuscript with the assistance of Patricia Scholz and Till Ischebeck. 


\title{
A GC-MS-based method of water extracted pectin monosaccharide analysis.
}

Patricia Scholz ${ }^{1}$, Till Ischebeck ${ }^{1}$, Athanas Guzha ${ }^{1}$

1 University of Goettingen, Albrecht-von-Haller-Institute for Plant Sciences, Department of Plant Biochemistry, Justus-von-Liebig Weg 11, D-37077 Goettingen, Germany.

Corresponding authors: tischeb@gwdg.de and athanas.guzha@uni-goettingen.de

\begin{abstract}
The plant cell wall consists of a complex mix of polysaccharides that undergo various modifications during a plant cell's lifetime. Cell wall modifications are induced in response to environmental changes and physiological development needs. Identification of changes occurring in these cell walls is critical to get a better understanding of the link between cell wall composition and function and requires suitable methods to determine cell wall composition. Here, we describe a GC-MS-based method to measure the monosaccharide composition of pectin derived sugars. We used this method for fast and straightforward monosaccharide composition analysis of pectin derived from different organs including leaves, seeds and fruit peel as proof of concept. We provide an improved GC-MS method as a useful tool to study pectin composition from various sources.
\end{abstract}


All plants have cell walls that are important for mechanical support, cell-to-cellcommunication, plant morphogenesis and plant defence responses. To fulfil these diverse functions, plant cell walls are composed of an intricate assembly of carbohydrates, proteins and phenolic compounds, varying between different cell types and developmental stages (Carpita and Gibeaut, 1993; Freshour et al., 2003; Knox et al., 1990). Primary cell walls consist up to $90 \%$ of polysaccharides like cellulose, hemicelluloses and pectin (Caffall and Mohnen, 2009; Höfte and Voxeur, 2017; Pettolino et al., 2012). Cellulose and hemicelluloses form a strong network that is embedded into the amorphous matrix formed by pectin (Carpita and Gibeaut, 1993). Pectin consists of several different polysaccharide domains, each characterised by a high content of galacturonic acid linked in O1- and O4-positions. The most abundant and least structurally complex pectic polysaccharide, homogalacturonan (HG), has a backbone of $\alpha(1 \rightarrow 4)$-linked D-galacturonic acid $(G a l A)$ monomers. GalA monomers in a HG chain may be O-acetylated in $\mathrm{O} 2$ or $\mathrm{O} 3$ position; furthermore, GalA may be substituted with D-xylose in $\mathrm{O} 3$ position or D-apiofuranose in O2-/O3-position to form xylogalacturonan and apiogalacturonan, respectively (Anderson, 2016; Atmodjo et al., 2013; Mohnen, 2008; Caffall and Mohnen, 2009). Similar to HG, rhamnogalacturonan II (RG II) possesses a backbone formed by $a(1 \rightarrow 4)$-linked D-GalA monomers, however, it is substituted with four different structurally complex side chains ( Mohnen, 2008; Anderson, 2016; Voiniciuc et al., 2018). Unlike the other two pectic polysaccharides, rhamnogalacturonan I (RG I) has a backbone formed by the disaccharide repeat of [- $\alpha-D-G a l A-1,2-\alpha-L-R h a-1,4-]$. Depending on cell type and developmental states, the backbone carries different arabinan, galactan or arabinogalactan side chains (Mohnen, 2008; Anderson, 2016; Bidhendi and Geitmann, 2016).

Matrix polysaccharides of pectin are synthesised in the Golgi, transported in secretory vesicles and further modified in mura (Harholt et al., 2010; Mohnen, D., Bar-Peled, M. and Somerville, 2008; Ridley et al., 2001). Modifications in the cell wall occur due to enzymes localised in the apoplast and are often linked to responses to pathogen attack (Körner et al., 2009; Wang et al., 2017). To monitor changes and modifications of pectin it is therefore necessary to develop methods to identify alterations of its biochemical composition. Furthermore, description of the various enzymes involved in pectin biosynthesis and their respective Arabidopsis mutants requires reliable analysis 
of pectin composition. However, the insoluble yet amorphous properties of pectic compounds makes a detailed characterisation of their structure in mura a challenging task. The use of different fluorescent probes, monoclonal antibodies and sugar analogues derived from click chemistry for monitoring of cell wall pectin has been described, however, it gives limited information about the relative amounts of the different monosaccharides (Bethke and Glazebrook, 2019; Hoogenboom et al., 2016; Voiniciuc et al., 2018). Complete biochemical analysis of cell wall components combines analysis of the components, their respective abundance and the linkages they undergo in the cell wall. Full linkage analysis is a time-consuming process, as it requires methylation of cell wall samples for the differentiation of free hydroxyl groups ahead of cell wall hydrolysis and component analysis (Biswal et al., 2017; York et al., 1986; Pettolino et al., 2012). Additionally, for several purposes such as monitoring of pathogen induced cell wall remodelling, a composition analysis alone already provides the required information.

Pectin for analysis can derive from different plant sources. Cell wall samples, e.g. of leaves, are often prepared in form of alcohol insoluble residue (AIR) (Gille et al., 2009; Pettolino et al., 2012; Biswal et al., 2017). An established alternative for the analysis of $R G I$ is seed coat mucilage, an extracellular matrix that is rapidly extruded from mucilage secretory cells of Arabidopsis seeds upon hydration (Arsovski et al., 2010; Voiniciuc et al., 2015). Independent of the source, pectin polysaccharides are subsequently hydrolysed into their respective monosaccharides. These can then be quantified by a variety of methods including colorimetric assays, GC-MS- and HPLC-based approaches (Willför et al., 2009; Pettolino et al., 2012; Biswal et al., 2017; Bethke and Glazebrook, 2019). For the use of GC-MS detection, hydrolysis products are further derivatised to be volatile enough for GC separation. Two main methods for volatilisation are described in the literature: reduction and acetylation to form alditol acetates or methanolysis of cell wall samples followed by trimethylsilylation (York et al., 1986; Merkle and Poppe, 1994; Biswal et al., 2017). Derivatisation to alditol acetates will impair the detection of uronic acids, as the carboxyl-group is reduced in the reduction step. For the analysis of pectin composition, the analysis of alditol acetates is therefore unsuitable. However, methanolysis of cell wall samples is a timeconsuming processing requiring incubation times of 15 hours or more. Additionally, methanolysis results in several different analytes formed from one monosaccharide, 
leading to several signals in the GC chromatogram and the methylation of monosaccharides complicates absolute quantification of hydrolysed sugars (York et al., 1986; Biswal et al., 2017).

Here we describe a fast method for GC-MS-based compositional analysis of cell wall pectin extracted from cell wall samples by water extraction using a modified hydrolysis and derivatisation protocol. TFA is used as hydrolysing agent, which greatly reduces hydrolysis time and does not alter the chemical nature of the monosaccharide building blocks. These are then easily derivatised with methoxyamine (MOX) and N-Methyl-N(trimethylsilyl)trifluoroacetamide (MSTFA), enabling compositional analysis of pectic samples in 2-3 days.

\section{Results and Discussion}

Monosaccharide composition of Sigma-Aldrich pectin from citrus.

To test the efficiency and accuracy of our method, commercially available pectin of citrus peels was hydrolysed, derivatised with MOX and MSTFA, and analyzed on the GC-MS. Pectin consists of various sugars including galacturonic acid, galactose, rhamnose, arabinose, fucose, xylose and in minor amounts glucuronic acid (Vincken et al., 2003; Voragen et al., 2009). Other sugars from cell wall polysaccharides such as glucose and mannose can also be detected. All the expected sugars except glucuronic acid could be separated with GC and the expected analytes could be detected by MS (Figure 1A). Some of these monosaccharides are pairs of diastereomers including arabinose/xylose, rhamnose/fucose, galactose/glucose/mannose, and galacturonic acid/glucuronic acid. As of this, they generate similar mass spectra, making MS-based identification a challenge. To counteract this challenge, the individual sugars were obtained as pure substances and the retention times of their derivatives recorded. Derivatisation resulted in the formation of two isomeric analytes in a stable stoichiometric ratio. Consequently, for most monosaccharides two peaks with characteristic retention times and a fixed ratio to each other could be detected. Based on differences in retention time, the individual sugars could be clearly separated.

The most abundant monosaccharide in the pectin of citrus peels was galacturonic acid that contributed $79.5 \%$ to the total amount of monosaccharides (Figure 1B). This is 
consistent with the product description of at least $74 \%$ galacturonic acid content. The most abundant neutral sugars that we observed were galactose, arabinose and rhamnose. Our results are consistent with established pectin structure of a galacturonic acid derived backbone and galactan and arabinan side chains (Mohnen, 2008; Zdunek et al., 2014).

A

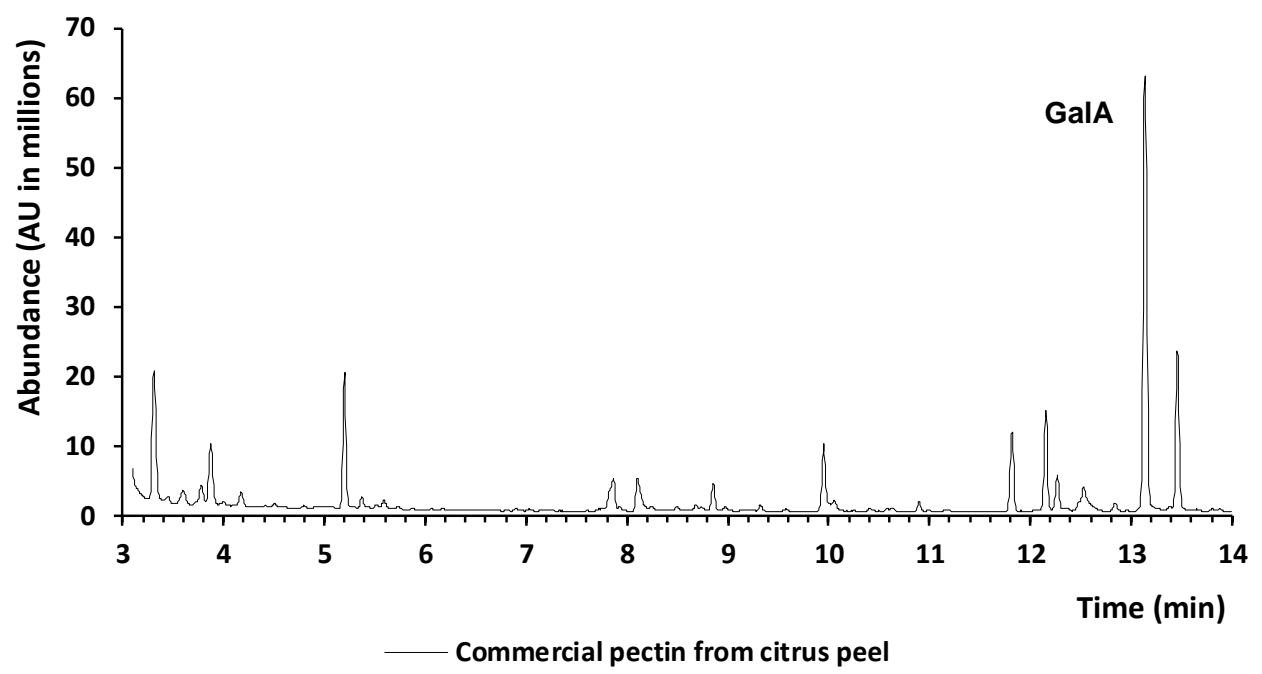

B Monosaccharide composition (\%) of citrus peel pectin

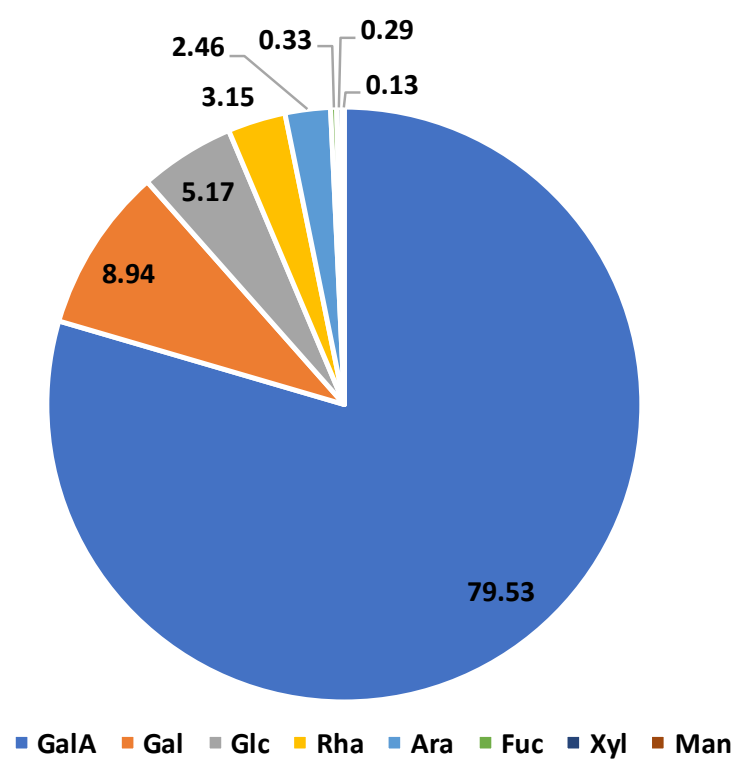

Figure 1: Monosaccharide composition (\%) of commercial pectin from citrus peel (Sigma-Aldrich).

(A) Chromatogram of GC-MS run showing the expected analytes eluting within the first 14 minutes. (B) Galacturonic acid (GalA), galactose (Gal), glucose (Glc), 
rhamnose (Rha), arabinose (Ara), fucose (Fuc), xylose (Xyl), mannose (Man) could all be detected and quantified. Data represents averages of 4 replicates.

Mucilage monosaccharide composition varies between Arabidopsis ecotypes and mutants

The relative composition of water-extracted mucilage from previously described Arabidopsis lines was investigated to make a comparison with already published data. Mucilage was extracted from Col-0, Ws and a mutant, $b x / 1$, in the Ws background which was shown to have higher arabinose levels in its mucilage (Arsovski et al., 2009). Mucilage, being a type of cell wall with all the basic components such as pectin, cellulose, hemicellulose and cell wall proteins, provides an ideal model system to analyse cell wall variances in different ecotypes and modifications due to activity of 


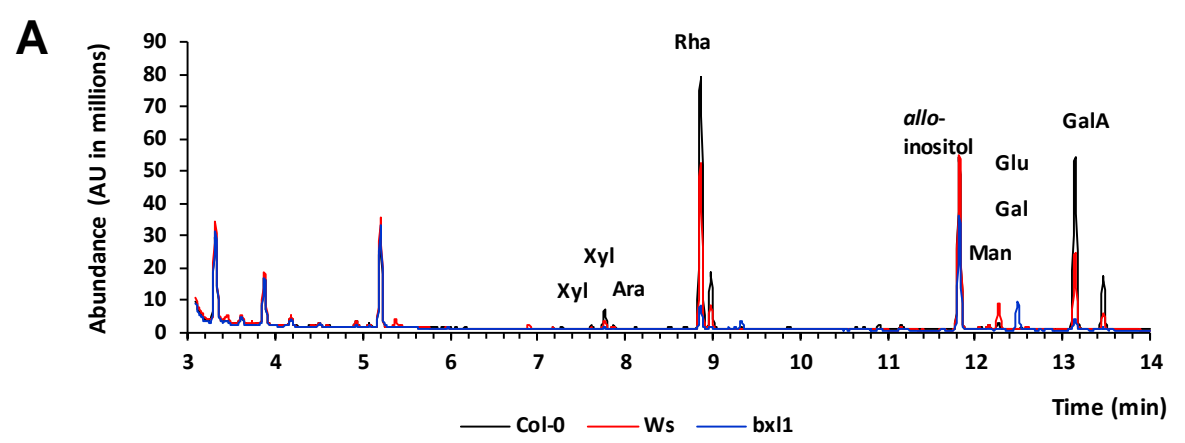

B

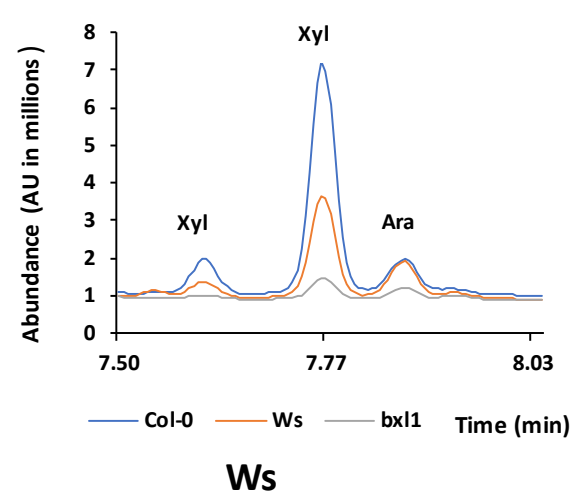

D

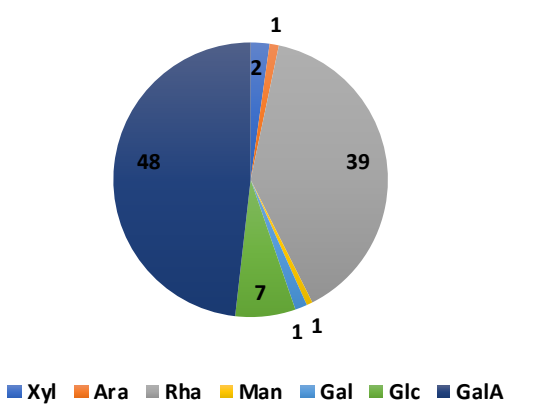

C

Col-0

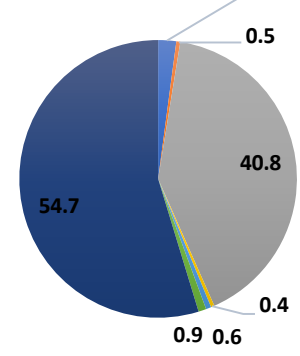

Xyl $\square$ Ara $\square$ Rha $\square$ Man $\square$ Gal $\square$ Glc $\square$ GalA

bxl1

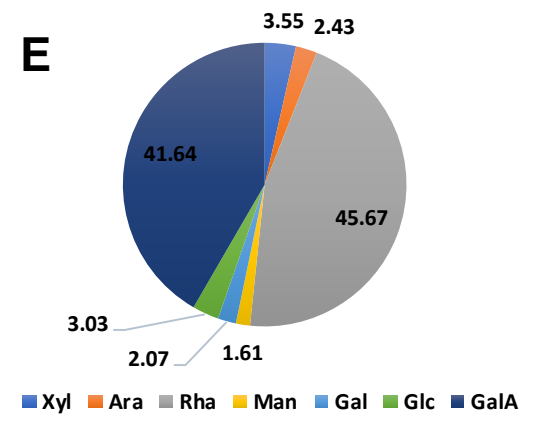

Figure 2: Chromatograms and monosaccharide composition (\%) of different Arabidopsis ecotypes and mutants.

(A) Chromatograms indicating mucilage pectin sugars eluting within the first 14 minutes. (B) Enlarged section of the chromatogram showing the elution of xylose and arabinose in Col-0, Ws and bx/1 mutant. (C-E) Mucilage monosaccharide composition (\%) of Col-0 (C) Ws (D) and bx/1 (E) data represents the average of 4 replicates.

various proteins (Western et al., 2000; Arsovski et al., 2010). All the expected sugars could be detected with the exception of the minor sugar fucose that was probably below the detection level. Rhamnose and galacturonic acid were the dominant sugars (Figure 2A \& B) with relative amounts of $39.3 \%$ (rhamnose) and $48.2 \%$ (galacturonic acid) in Ws and $45.7 \%$ (rhamnose) and $41.6 \%$ (galacturonic acid) in bxl1. This is consistent with the fact that mucilage consists mostly of the pectin RG I, which has an 
alternating rhamnose-galacturonic acid backbone (Haughn and Western, 2012). BXL1 is a bifunctional $\beta$-D-xylosidase/ $\alpha-L$-arabinofuranosidase, so the chromatograms generated with regard to xylose and arabinose separation were of interest (Figure 2C). After calculating monosaccharide composition, we could observe a 2-fold increase in arabinose content in the mucilage of $b x / 1$ compared to the Ws wild type (Figure 2D). This was consistent with previously published data (Arsovski et al., 2009). The mucilage monosaccharide compositions obtained from Col-0 showed galacturonic acid and rhamnose contributing $54.7 \%$ and $40.8 \%$ of total monosaccharides, respectively. The other side chain sugars present in RG I, derived from galactose and arabinose, could also be detected and are in proportions similar to other publications that used high-performance anion-exchange chromatography with pulsed amperometric detection to measure the sugars (Voiniciuc and Günl, 2016).

\section{Analysis of water-soluble pectin from leaf alcohol insoluble residue.}

Water-soluble pectin was extracted from the alcohol insoluble residue prepared from Arabidopsis Col-0 leaves. It is known that the extraction method used in pectin extraction affects its composition (Rose et al., 1998). Cell wall disintegration due to heating allows for the extraction of water soluble pectin, whilst acid and enzyme addition allows for the extraction of insoluble pectin that tightly interacts with other cell wall polysaccharides (Assoi et al., 2014). Monosaccharide analysis of the water soluble pectin indicated that galacturonic acid was the dominant monosaccharide (Figure $3 \mathrm{~A} \& \mathrm{~B}$ ), constituting $72 \%$ of the extracted monosaccharides (Figure $3 \mathrm{C}$ ). Our data is consistent with a previously described galacturonic acid content of ca. $70 \%$ in pectin (Mohnen, 2008). The extracted pectin also confirmed the presence of neutral sugars, with the most abundant being galactose, arabinose and rhamnose. The monosaccharide profile obtained indicates that the extracted component from the AIR is predominantly pectin, given the high abundance of galacturonic acid and rhamnose, and that the galactose and arabinose are mostly from pectin side chains (Zdunek et al., 2014; Vincken et al., 2003). In water-extracted pectin of leaf cell walls, there is normally an abundance of glucose derived from starch, and to minimise the amount of glucose detected, plants can be incubated in the dark for 48 hours. However, for monosaccharide composition analysis of pectin, glucose does 
not present a problem because it is not a component of pectin and can be ignored. It is also clearly separated from its diastereomer galactose so it does not interfere with absolute quantification.
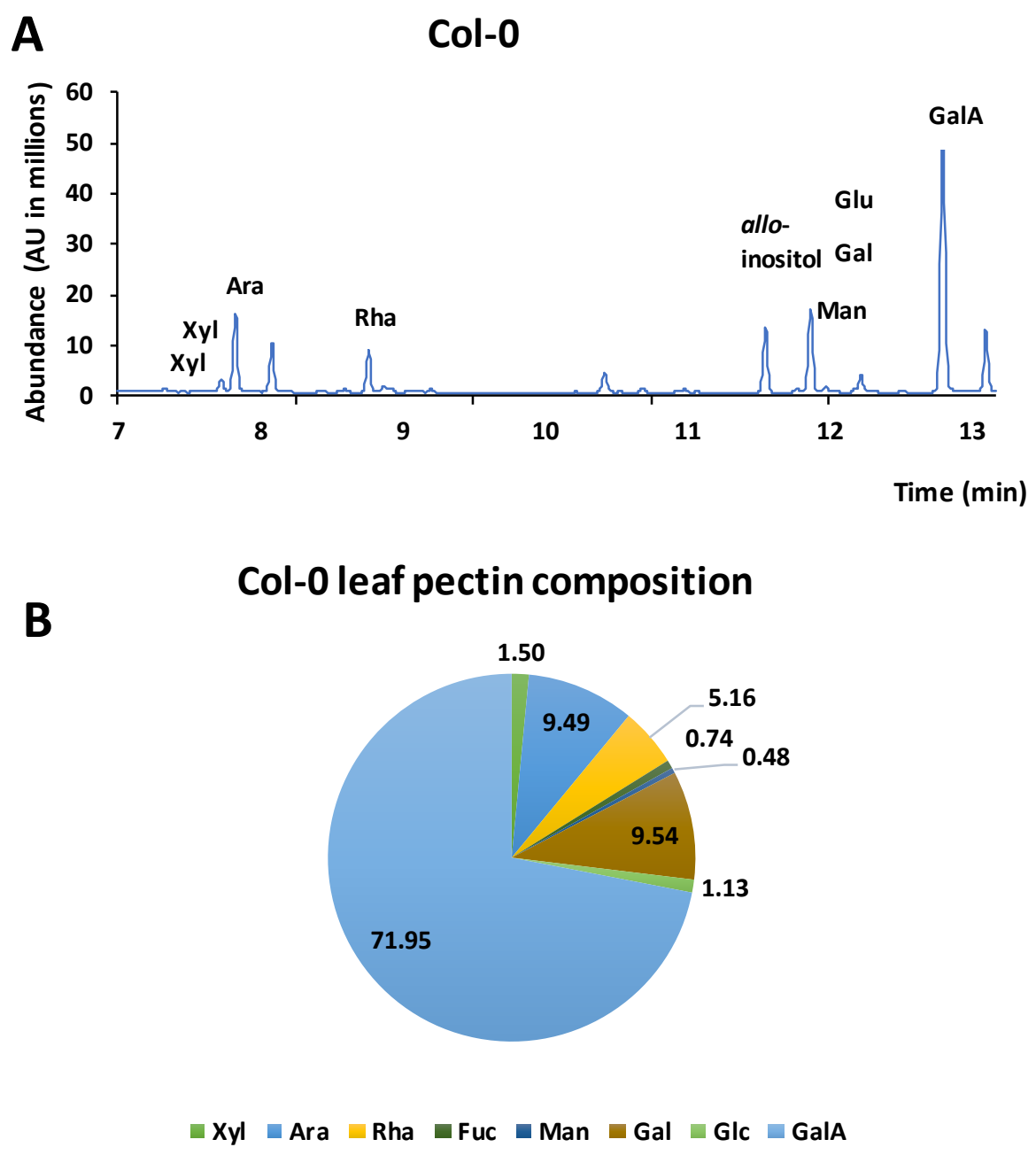

Figure 3: Chromatogram and monosaccharide composition of water extracted pectin from leaf AIR.

(A) The chromatograms generated from GC-MS runs of water extracted pectin from Arabidopsis leaf AIR (A \& B). (B) The monosaccharide composition (\%) of the various sugars from leaf pectin analysed by GC-MS. The values represent means of 4 biological replicates. 
Table 1: Comparative summary of mucilage monosaccharide compositions obtained with GC-MS analysis versus published data. All values are given as percentage of total monosaccharide content.

\begin{tabular}{|l|l|l|l|l|l|l|}
\hline & $\begin{array}{l}\text { Col-0 } \\
\text { mucilage }\end{array}$ & $\begin{array}{l}\text { Col-0- } \\
\text { mucilage } \\
\text { (Voiniciuc } \\
\text { and Günl, } \\
2016)\end{array}$ & $\begin{array}{l}\text { Ws } \\
\text { mucilage }\end{array}$ & $\begin{array}{l}\text { Ws } \\
\text { mucilage } \\
\text { (Arsovski et } \\
\text { al., 2009) } \\
\text { Ammonium } \\
\text { oxalate } \\
\text { extraction }\end{array}$ & $\begin{array}{l}\text { bx/1 } \\
\text { mucilage }\end{array}$ & $\begin{array}{l}\text { bxl1 } \\
\text { mucilage } \\
\text { (Arsovski et } \\
\text { al., 2009) } \\
\text { Ammonium } \\
\text { oxalate } \\
\text { extraction }\end{array}$ \\
\hline Xylose & 2.1 & 3.9 & 2.2 & 3.7 & 3.6 & 3.3 \\
\hline Arabinose & 0.5 & 1.8 & 1.1 & 0.7 & 2.4 & 1.3 \\
\hline Rhamnose & 40.8 & 41.7 & 39.3 & 49.5 & 45.7 & 49.7 \\
\hline Fucose & 0 & & 0 & tr & 0 & tr \\
\hline Mannose & 0.4 & 0.9 & 0.7 & 1 & 1.6 & 1.2 \\
\hline Galactose & 0.6 & 1.6 & 1.4 & 1.8 & 2.1 & 1.9 \\
\hline Glucose & 0.9 & 1.6 & 7.1 & 2.3 & 3.0 & 2.1 \\
\hline $\begin{array}{l}\text { Galacturonic } \\
\text { acid }\end{array}$ & 54.7 & 48 & 48.2 & 40.9 & 41.6 & 40.4 \\
\hline
\end{tabular}

\section{Conclusion}

The GC-MS method we developed allowed us to quantify monosaccharide compositions of pectin extracted from leaves, seed mucilage and commercial pectin with reasonable accuracy comparing to what has been published before using other techniques. All nine monosaccharides are detected, so acidic and neutral sugars can be analysed in parallel. Due to the MS-based detection, mass spectra can be used for partial confirmation of monosaccharide identity. The use of TFA as hydrolysing reagent greatly reduces hydrolysis time compared to acidic methanolysis, thus sample preparation up to the first derivatisation step can be carried out in one day followed by GC-MS analysis the next day. Furthermore, as monosaccharides are not modified with methyl groups, absolute quantification is easily achieved by calculating area-response factors with the help of external standards. In summary, we provide an improved method for pectin analysis for comparative purposes between different lines or treatments.

\section{Reagents}


- Distilled water

- Liquid nitrogen

- Nitrogen gas

- Ethanol

- Methanol

- Chloroform

- Acetone

- MOX (Methoxyamine hydrochloride, Sigma-Aldrich 226904)

- MSTFA (N-Methyl-N-(trimethylsilyl)trifluoroacetamide, Sigma-Aldrich 69479)

- Acetonitrile (Sigma-Aldrich 271004)

- TFA (Trifluoroacetic acid, Sigma-Aldrich 302031

- Pyridine Anhydrous (Sigma-Aldrich 270970)

- allo-inositol (Sigma-Aldrich 468088)

- L-(-)- Fucose (Sigma-Aldrich F2252)

- D-Glucuronic acid (Sigma-Aldrich G8645)

- D-(+)-Galacturonic acid (Sigma-Aldrich 48280)

- L-(+)-Arabinose (Fluka-Biochemika 10845)

- L-(+)-Rhamnose (CARL-ROTH 4655.2)

- D-(+)-Mannose (Merck 5984)

- D-(+)-Xylose (Merck 8692)

- D-(+)-Galactose (CARL-ROTH 4987.2)

- D-(+)-Glucose (CARL-ROTH 6780.2)

- Citrus pectin (Sigma-Aldrich P9135)

\section{Equipment}

- Fume hood

- Retsch ball mill

- Mortar and pestle

- Heating block with shaker for 2 ml Eppendorf tubes (HLC HTM 130)

- Heating block for Duran tubes

- Rotary shaker

- Digital scale 
- GC-MS system

- Heat resistant test tube rack

- Test tube heating block/oven

- Vortexer

- Duran tubes

- Glass Pasteur pipettes

- Acid resistant pipettes

- 2 ml GC-MS-Vials + inserts

- Glass beads $3 \mathrm{~mm}$

\section{Procedures}

AIR analysis

Alcohol Insoluble residue preparation

The method used in this part of protocol is adapted from Gille et al., (2009) and Bethke et al., (2014) and involves the following steps:

1. Plants for analysis are incubated for $48 \mathrm{~h}$ in the dark. Incubation in the dark is an easy way to reduce the starch content, and minimize glucose overload.

2. Organs required for analysis (Leaves, stems, roots, whole rosettes) are flash frozen in liquid nitrogen, and pulverized using mortar and pestle. Grinding of material is a crucial step to prevent contamination of wall material with cytosolic derived sugars.

3. Pulverized material (up to $100 \mathrm{mg}$ ) is placed in $2 \mathrm{~mL}$ Eppendorf tube.

4. AIR is extracted by washing ground material in $1,5 \mathrm{~mL} 70 \%(\mathrm{v} / \mathrm{v})$ ethanol.

5. The supernatant is removed by centrifugation for $10 \mathrm{~min}$ at $14,000 \mathrm{rpm}$.

6. Repeat Ethanol wash as described above.

7. Carry out three washes with $1 \mathrm{~mL}$ chloroform/ methanol $1: 1(\mathrm{v} / \mathrm{v})$, with the supernatant obtained by centrifugation at $14000 \mathrm{rpm}$ for 10 minutes discarded after each wash. At the end of the last washing step plant material derived from chlorophyll containing tissue should have a dull grey to off-white color.

8. Wash AIR in $1 \mathrm{ml}$ acetone, and centrifuge at $14000 \mathrm{rpm}$ for 10 minutes. 
9. Discard the supernatant and air dry the AIR at room temperature overnight.

\section{AIR pectin analysis}

1. Prepare 4 replicates for each genotype. Weigh $2 \mathrm{mg}$ of AIR for each replicate and place in a $2 \mathrm{~mL}$ Eppendorf tube. Record the exact weight of AIR placed in Eppendorf tube. Use plants grown at the same time and under the same conditions.

2. Add $1.4 \mathrm{~mL}$ of $\mathrm{ddH}_{2} \mathrm{O}$ to the AIR.

3. Add 2 glass bead $(3 \mathrm{~mm})$ and homogenise using a Retsch ball mill at $30 \mathrm{~Hz}$ for 1.5 mins

4. For hot water extraction of pectin, incubate the samples for 2 hours on a shaker at $90^{\circ} \mathrm{C}$.

5. Allow debris to settle and remove $1 \mathrm{~mL}$ of supernatant to a Duran glass tube.

6. Dry under $\mathrm{N}_{2}$ stream, heating the samples to $40^{\circ} \mathrm{C}$.

7. Add $300 \mu \mathrm{L}$ of $2 \mathrm{M}$ TFA.

8. For TFA hydrolysis, incubate at $121^{\circ} \mathrm{C}$ for 1 hour. Do this step under a fume-hood.

9. Evaporate the TFA under $\mathrm{N}_{2}$ stream at room temperature under a fume-hood.

10. Extract hydrolysis products with $500 \mu \mathrm{L} \mathrm{ddH}_{2} \mathrm{O}$ plus $100 \mu \mathrm{L}$ of $0.05 \mathrm{mg} / \mathrm{mL}$ allo-inositol.

11. For GC-MS: Evaporate $20 \mu \mathrm{L}$ under $\mathrm{N}_{2}$ stream. The remaining hydrolysis products can be stored for long term at $-20^{\circ} \mathrm{C}$.

12. Derivatise dried sample with $15 \mu \mathrm{L} 30 \mathrm{mg} / \mathrm{mL}$ MOX in anhydrous pyridine at room temperature over-night.

13. Add $30 \mu \mathrm{L}$ MSTFA and analyse on GC-MS after incubation of 1-6 hours.

Mucilage analysis

1. Prepare 4 replicates for each genotype. Weigh $2 \mathrm{mg}$ of seeds for each replicate and place in a $2 \mathrm{~mL}$ Eppendorf tube. Record the exact weight. 
2. Add $1.4 \mathrm{~mL}$ of $\mathrm{dd} \mathrm{H}_{2} \mathrm{O}$ to the Eppendorf tube

3. Place on rotary shaker and shake for 2 hours to extract the mucilage.

4. Allow the seeds to settle and transfer $1 \mathrm{~mL}$ of supernatant to a Duran glass tube.

5. Dry under $\mathrm{N}_{2}$ stream, heating the samples to $40^{\circ} \mathrm{C}$.

6. Add $300 \mu \mathrm{L}$ of $2 \mathrm{M}$ TFA

7. For TFA hydrolysis, incubate at $121^{\circ} \mathrm{C}$ for 1 hour . Do this step under a fume hood.

8. Evaporate the TFA under $\mathrm{N}_{2}$ stream at room temperature under a fume hood.

9. Extract hydrolysis products with $500 \mu \mathrm{L} \mathrm{ddH}_{2} \mathrm{O}$ plus $100 \mu \mathrm{L}$ of $0.05 \mathrm{mg} / \mathrm{mL}$ allo-inositol.

10. For GC-MS: Evaporate $20 \mu \mathrm{L}$ under $\mathrm{N}_{2}$ stream. The remaining hydrolysis products can be stored for long term at $-20^{\circ} \mathrm{C}$.

11. Derivatise dried sample with $15 \mu \mathrm{L} 30 \mathrm{mg} / \mathrm{mL}$ MOX in anhydrous pyridine at room temperature over-night.

12. Add $30 \mu \mathrm{L}$ MSTFA and analyse on GC-MS after incubation of 1-6 hours. Samples should be measured 1-6 hours after addition of MSTFA.

\section{GC-MS analysis}

Samples were analysed with the 7890B GC-System coupled to a 5977B MSD quadrupole set-up from Agilent Technologies. GC-separation was achieved on a HP-5 column (Agilent Technologies) using the following temperature gradient: $150^{\circ} \mathrm{C}$ for 2 $\min , 5 \mathrm{~K} / \mathrm{min}$ gradient for $20 \mathrm{~min}, 15 \mathrm{~K} / \mathrm{min}$ to a final temperature of $320^{\circ} \mathrm{C}$ and $3 \mathrm{~min}$ hold at $320^{\circ} \mathrm{C}$. For each run, $1 \mu \mathrm{L}$ of the derivatised sample was injected. Compounds were identified by a combination of retention times compared to external standards and MS-spectra of electron impact mass spectrometry.

Prepared mucilage samples were quantified relative to the internal standard allo-inositol. In parallel runs, monosaccharide standards of different concentration were used to determine response factors for area-to-molar amount conversion 
allowing absolute quantification. Sugars were normalized to the mass of pectin, seeds or AIR used for extraction.

Results were analyzed using the program "Instrument \#1 Data Analysis" from Agilent Technologies. Peaks were identified with the help of standards from the cell wall monosaccharides arabinose, fucose, galactose, galacturonic acid, glucose, mannose, rhamnose and xylose.

For quantification, area-to-molar amount response factors had to be established for each cell wall monosaccharide. To that end, defined molar amounts (1-3 nmol) of each monosaccharide were derivatised and measured like pectin samples. Three replicates of each concentration were analysed. Peak areas of target ions from the identified compounds were integrated and normalized to the peak area of the internal standard allo-inositol. The normalized peak areas were then plotted against the molar amounts. From the resulting calibration curve the response factors were determined which could then be used to calculate the molar amount of cell wall monosaccharides present in mucilage samples.

\section{Acknowledgements}

This work was funded by the German Research Foundation (DFG, IRTG 2172 PRoTECT to T.I.) and the Studienstiftung des deutschen Volkes (stipend to P.S.). We are grateful to Dr Gillian Dean and Dr Kresimir Sola for their ideas and suggestions and Franziska Kretzschmar for proofreading the manuscript.

\section{Author contributions}

P.S. and A.G. performed the experiments and wrote the manuscript. T.I. designed the project and assisted with the data analysis.

\section{References}

Anderson, C.T. (2016). We be jammin': an update on pectin biosynthesis, trafficking and dynamics. J. Exp. Bot. 67: 495-502.

Arsovski, A.A., Haughn, G.W., and Western, T.L. (2010). Seed coat mucilage cells of Arabidopsis thaliana as a model for plant cell wall research. Plant Signal. 
Behav. 5: 796-801.

Arsovski, A.A., Popma, T.M., Haughn, G.W., Carpita, N.C., McCann, M.C., and Western, T.L. (2009). AtBXL1 encodes a bifunctional beta-D-xylosidase/alpha-Larabinofuranosidase required for pectic arabinan modification in Arabidopsis mucilage secretory cells. Plant Physiol. 150: 1219-34.

Assoi, S., Konan, K., Walker, L.T., Holser, R., Agbo, G.N., Dodo, H., and Wicker, L. (2014). Functionality and yield of pectin extracted from Palmyra palm (Borassus aethiopum Mart) fruit. LWT - Food Sci. Technol. 58: 214-221.

Atmodjo, M.A., Hao, Z., and Mohnen, D. (2013). Evolving Views of Pectin Biosynthesis. Annu. Rev. Plant Biol. 64: 747-779.

Bethke, G. and Glazebrook, J. (2019). Measuring Pectin Properties to Track Cell Wall Alterations During Plant-Pathogen Interactions. Methods Mol. Biol. 1991: $55-60$.

Bethke, G., Grundman, R.E., Sreekanta, S., Truman, W., Katagiri, F., and Glazebrook, J. (2014). Arabidopsis PECTIN METHYLESTERASEs contribute to immunity against Pseudomonas syringae. Plant Physiol. 164: 1093-107.

Bidhendi, A.J. and Geitmann, A. (2016). Relating the mechanics of the primary plant cell wall to morphogenesis. J. Exp. Bot. 67: 449-461.

Biswal, A.K., Tan, L., Atmodjo, M.A., DeMartini, J., Gelineo-Albersheim, I., Hunt, K., Black, I.M., Mohanty, S.S., Ryno, D., Wyman, C.E., and Mohnen, D. (2017). Comparison of four glycosyl residue composition methods for effectiveness in detecting sugars from cell walls of dicot and grass tissues. Biotechnol. Biofuels 10.

Caffall, K.H. and Mohnen, D. (2009). The structure, function, and biosynthesis of plant cell wall pectic polysaccharides. Carbohydr. Res. 344: 1879-1900.

Carpita, N.C. and Gibeaut, D.M. (1993). Structural models of primary cell walls in flowering plants: consistency of molecular structure with the physical properties of the walls during growth. Plant J. Cell Mol. Biol. 3: 1-30. 
Freshour, G., Bonin, C.P., Reiter, W.-D., Albersheim, P., Darvill, A.G., and Hahn, M.G. (2003). Distribution of fucose-containing xyloglucans in cell walls of the mur1 mutant of Arabidopsis. Plant Physiol. 131: 1602-1612.

Gille, S., Hansel, U., Ziemann, M., and Pauly, M. (2009a). Identification of plant cell wall mutants by means of a forward chemical genetic approach using hydrolases. Proc. Natl. Acad. Sci. 106: 14699-14704.

Gille, S., Hänsel, U., Ziemann, M., and Pauly, M. (2009b). Identification of plant cell wall mutants by means of a forward chemical genetic approach using hydrolases. Proc. Natl. Acad. Sci. U. S. A. 106: 14699-704.

Harholt, J., Suttangkakul, A., and Vibe Scheller, H. (2010). Biosynthesis of Pectin. Plant Physiol. 153: 384-395.

Haughn, G.W. and Western, T.L. (2012). Arabidopsis Seed Coat Mucilage is a Specialized Cell Wall that Can be Used as a Model for Genetic Analysis of Plant Cell Wall Structure and Function. Front. Plant Sci. 3: 64.

Höfte, H. and Voxeur, A. (2017). Plant cell walls. Curr. Biol. CB 27: R865-R870.

Hoogenboom, J., Berghuis, N., Cramer, D., Geurts, R., Zuilhof, H., and Wennekes, T. (2016). Direct imaging of glycans in Arabidopsis roots via click labeling of metabolically incorporated azido-monosaccharides. BMC Plant Biol. 16: 220 .

Knox, J.P., Linstead, P., King, J., Cooper, C., and Roberts, K. (1990). Pectin esterification is spatially regulated both within cell walls and between developing tissues of root apices. Planta 181.

Körner, E., von Dahl, C.C., Bonaventure, G., and Baldwin, I.T. (2009). Pectin methylesterase NaPME1 contributes to the emission of methanol during insect herbivory and to the elicitation of defence responses in Nicotiana attenuata. J. Exp. Bot. 60: 2631-40.

Merkle, R.K. and Poppe, I. (1994). Carbohydrate composition analysis of glycoconjugates by gas-liquid chromatography/mass spectrometry. In Methods in 
Enzymology (Elsevier), pp. 1-15.

Mohnen, D., Bar-Peled, M. and Somerville, C. (2008). Biosynthesis of Plant Cell Walls. In Biomass Recalcitrance, M. Himmel, ed (Blackwell Publishing, Oxford), pp. 94-187.

Mohnen, D. (2008). Pectin structure and biosynthesis. Curr. Opin. Plant Biol. 11: 266277.

Pettolino, F.A., Walsh, C., Fincher, G.B., and Bacic, A. (2012). Determining the polysaccharide composition of plant cell walls. Nat. Protoc. 7: 1590-1607.

Ridley, B.L., O'Neill, M.A., and Mohnen, D. (2001). Pectins: structure, biosynthesis, and oligogalacturonide-related signaling. Phytochemistry 57: 929-67.

Rose, J.K.C., Hadfield, K.A., Labavitch, J.M., and Bennett, A.B. (1998). Temporal Sequence of Cell Wall Disassembly in Rapidly Ripening Melon Fruit. Plant Physiol. 117: 345-361.

Vincken, J.-P., Schols, H.A., Oomen, R.J.F.J., Beldman, G., Visser, R.G.F., and Voragen, A.G.J. (2003). Pectin - the Hairy Thing. In Advances in Pectin and Pectinase Research (Springer Netherlands: Dordrecht), pp. 47-59.

Voiniciuc, C. and Günl, M. (2016). Analysis of Monosaccharides in Total Mucilage Extractable from Arabidopsis Seeds. Bio-Protocol 6.

Voiniciuc, C., Pauly, M., and Usadel, B. (2018). Monitoring Polysaccharide Dynamics in the Plant Cell Wall. Plant Physiol. 176: 2590-2600.

Voiniciuc, C., Yang, B., Schmidt, M., Günl, M., and Usadel, B. (2015). Starting to Gel: How Arabidopsis Seed Coat Epidermal Cells Produce Specialized Secondary Cell Walls. Int. J. Mol. Sci. 16: 3452-3473.

Voragen, A.G.J., Coenen, G.-J., Verhoef, R.P., and Schols, H.A. (2009). Pectin, a versatile polysaccharide present in plant cell walls. Struct. Chem. 20: 263-275.

Wang, X., Hou, S., Wu, Q., Lin, M., Acharya, B.R., Wu, D., and Zhang, W. (2017). IDL6-HAE/HSL2 impacts pectin degradation and resistance to Pseudomonas 
syringae pv tomato DC3000 in Arabidopsis leaves. Plant J. 89: 250-263.

Western, T.L., Skinner, D.J., and Haughn, G.W. (2000). Differentiation of Mucilage Secretory Cells of the Arabidopsis Seed Coat. Plant Physiol. 122: 345-356.

Willför, S., Pranovich, A., Tamminen, T., Puls, J., Laine, C., Suurnäkki, A., Saake, B., Uotila, K., Simolin, H., Hemming, J., and Holmbom, B. (2009). Carbohydrate analysis of plant materials with uronic acid-containing polysaccharides-A comparison between different hydrolysis and subsequent chromatographic analytical techniques. Ind. Crops Prod. 29: 571-580.

York, W.S., Darvill, A.G., McNeil, M., Stevenson, T.T., and Albersheim, P. (1986). Isolation and characterization of plant cell walls and cell wall components. In Methods in Enzymology (Elsevier), pp. 3-40.

Zdunek, A., Kozioł, A., Pieczywek, P.M., and Cybulska, J. (2014). Evaluation of the Nanostructure of Pectin, Hemicellulose and Cellulose in the Cell Walls of Pears of Different Texture and Firmness. Food Bioprocess Technol. 7: 3525-3535. 


\section{Supplementary figures}
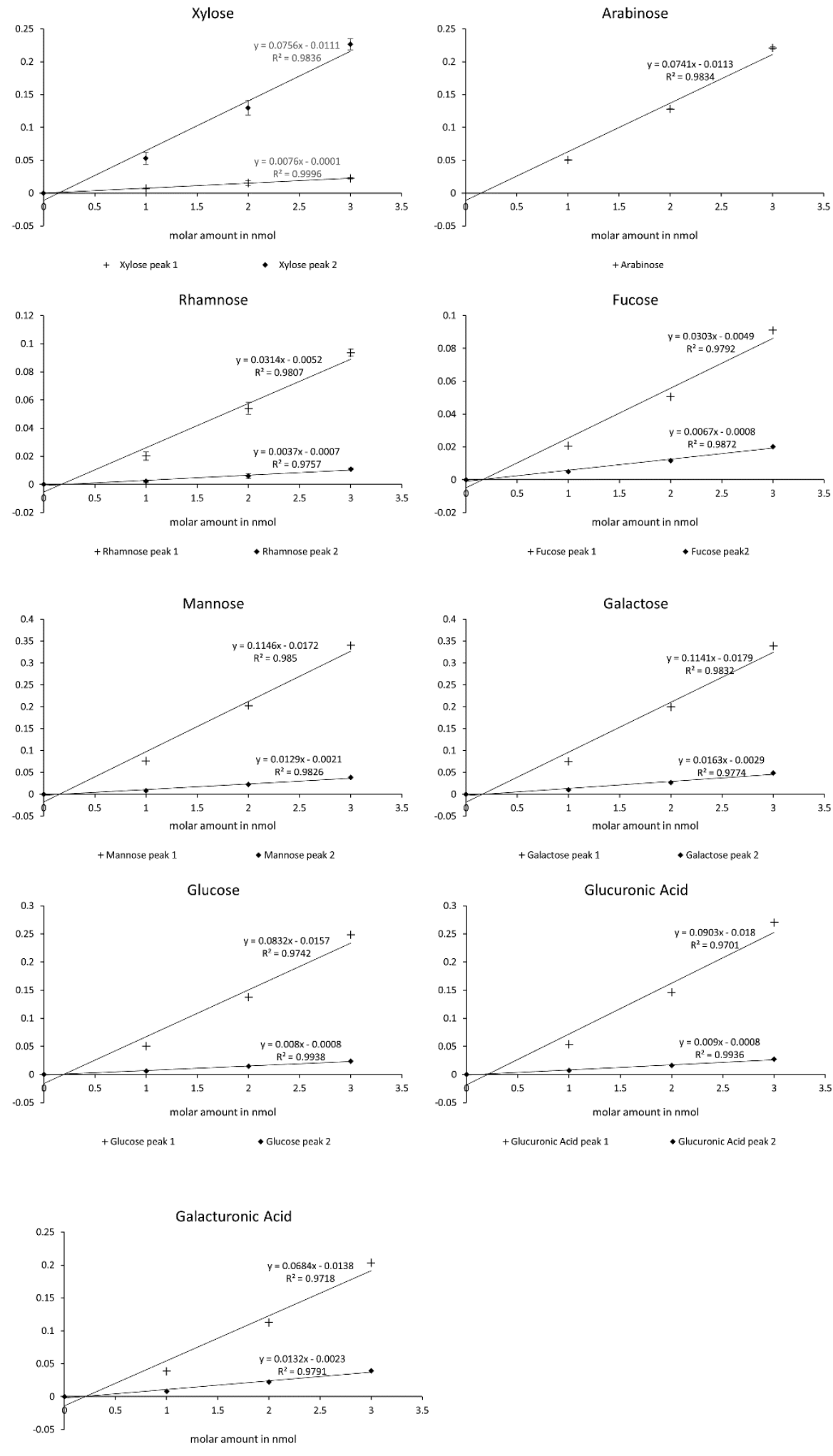

+Galacturonic Acid peak 1 • Galacturonic Acid peak 2 
Supplementary Figure 1: Calibration curves for the quantification of the different sugars

Supplementary Table 1: Sugar retention times

\begin{tabular}{|l|l|}
\hline Sugar & Retention time [min] \\
\hline Xylose peak 1 & 7.610 \\
\hline Xylose peak 2 & 7.760 \\
\hline Arabinose & 7.870 \\
\hline Rhamnose peak 1 & 8.860 \\
\hline Rhamnose peak 2 & 8.975 \\
\hline Fucose peak 1 & 9.020 \\
\hline Fucose peak 2 & 9.230 \\
\hline Mannose peak 1 & 12.050 \\
\hline Mannose peak 2 & 12.305 \\
\hline Galactose peak 1 & 12.150 \\
\hline Galactose peak 2 & 12.530 \\
\hline Glucose peak 1 & 12.265 \\
\hline Glucose peak 2 & 12.590 \\
\hline Glucuronic Acid peak 1 & 12.985 \\
\hline Glucuronic Acid peak 2 & 13.245 \\
\hline Galacturonic Acid peak 1 & 13.140 \\
\hline Galacturonic Acid peak 2 & 13.460 \\
\hline
\end{tabular}




\section{Manuscript B: Modification of pectin rhamnogalacturonan I contributes to plant immunity against Botrytis cinerea.}

This manuscript is being prepared for submission.

Individual contribution

Athanas Guzha cloned all the constructs used in this work. He was involved in the confocal microscopy work (Figure $1 \mathrm{~A}$ and B, Supplementary figure $1 \mathrm{~A}$ and $\mathrm{B}$ ). He also did the Arabidopsis transformations for mucilage rescue screens and the monosaccharide composition analysis (Figure 2 and 3). He did the gene expression analysis (Figure 4) and the Botrytis infection assays (Figure 6). Athanas Guzha wrote the manuscript with the help of other authors. 
Modification of pectin rhamnogalacturonan I contributes to plant immunity against Botrytis cinerea.

Athanas Guzha1, Robert McGee², Denise Harkten², Patricia Scholz ${ }^{1}$, Krzysztof Zienkiewicz ${ }^{1}$, Ivo Feussner ${ }^{1}$ Marcel Wiermer ${ }^{3}$, George Haughn ${ }^{2}$, Till Ischebeck ${ }^{1}$

${ }^{1}$ University of Goettingen, Albrecht-von-Haller-Institute for Plant Sciences, Department of Plant Biochemistry, Justus-von-Liebig Weg 11, D-37077 Goettingen, Germany.

${ }^{2}$ University of British Columbia, Department of Botany, Vancouver, BC V6T 1Z4, Canada

${ }^{3}$ University of Goettingen, Albrecht-von-Haller-Institute for Plant Sciences, RG Molecular Biology of Plant-Microbe Interactions, Julia-LermontowaWeg 3, D-37077 Goettingen, Germany.

Corresponding author: tischeb@gwdg.de

\section{ABSTRACT}

Plant cell walls constitute a barrier that plant pathogens have to breach to access contents of the plant cells. The primary cell wall of multicellular plants consists of three major classes of polysaccharides: cellulose, hemicellulose and pectin. Cellulose microfibrils are crosslinked by hemicelluloses and are surrounded by a pectic matrix. Cell wall proteins constitute a relatively minor component of cell walls but they play fundamental roles in maintenance of structural integrity as well as modification of the polysaccharide matrix during development and in response to environmental changes. Rhamnogalacturonan I (RG-I) is the second most abundant pectin found in primary cell walls and enzymes that modify it, such as beta-xylosidases/betaarabinosidases, are found in all plant cell walls. In Arabidopsis, a cell wall localised protein BETAXYLOSIDASE 4 (BXL4) belonging to a seven-member gene family BETAXYLOSIDASE (BXL), is induced upon infection with various pathogens and wounding in a jasmonoyl isoleucine (JA-lle) dependent manner. Ectopic expression of BXL4 in Arabidopsis seed coat epidermal cells suggests 
that like another member of the gene family ( $B X L 1)$, the protein it encodes has both xylosidase and arabinosidase activity and acts on RG-I. Bx/4 knockout mutants exhibit an increased abundance of arabinans, and have a compromised resistance to the necrotrophic pathogen Botrytis cinerea. Upon infection, the bx/4 knockout mutants show reduced levels of JA-Ile and camalexin accumulation. These data suggest that remodelling of the pectin RG-I could be an important component in plant immunity against $B$. cinerea, and might play a role in JA-Ile and camalexin accumulation.

\section{INTRODUCTION}

Plants are continuously exposed to a plethora of potential biotic threats such as herbivorous insects and phytopathogens, as well as constantly changing weather and climatic conditions. To help mitigate against these threats, plants have evolved various constitutive and inducible defences against the different biotic and abiotic perturbations.

Together with the cuticle, the plant cell wall forms an important barrier that pathogens have to breach to access cell contents (Vorwerk et al., 2004; Underwood, 2012; Engelsdorf et al., 2017). The importance of plant cell walls in plant immunity is demonstrated by the abundance of cell wall degrading enzymes (CWDEs) that pathogens secrete in order to successfully invade plant tissues (Glass et al., 2013; Quoc \& Bao Chau, 2017). Plant cell walls consist of a complex of polysaccharides where cellulose microfibrils are cross-linked by various hemicelluloses and embedded in a pectic matrix. Cellulose consists of (1-4)- $\beta$ linked D-glucose residues and is synthesised by cellulose synthases located in the plasma membrane (Somerville, 2006; Carpita, 2011). Hemicelluloses are a widely varied group of polysaccharides, with the most abundant in Arabidopsis being xyloglucan which is characterised by a $(1,4)$ - $\beta$-linked glucan regularly substituted with $\alpha-(1-6)$-xylosyl residues (Liepman et al., 2010; Scheller and Ulvskov, 2010). Cellulose and hemicelluloses are embedded in a pectin matrix.

Pectin is the most complex of all cell wall polysaccharides, and its biosynthesis involves at least 67 glycosyltransferases localised in the Golgi (Harholt et al., 2010) before it is exported to the apoplast. Pectin consists of four types of polysaccharides: Homogalacturonan, xylogalacturonan and rhamnogalacturonan II (RG-II), all 
characterised by the presence of a (1-4)- $\alpha$-D-galacturonic acid backbone, and rhamnogalacturonan I (RG-I), which has a backbone of alternating (1-2)- $\alpha-\mathrm{L}-$ rhamnose and (1-4)-a-D-galacturonic acid (O'Neill et al., 1990; Ridley et al., 2001; Mohnen, 2008; Mohnen, et al., 2008). RG-I is also characterised by arabinan, galactan and arabinogalactan side chains, and recently xylan side chains were also proposed to be present (Ralet et al., 2016). In Arabidopsis, pectin is the most abundant class of polysaccharide in the primary cell wall, and is important for the regulation of cell wall mechanical properties during growth and development. It also influences water imbibition of seeds, pollen tube growth, leaf and flower abscission, fruit ripening, and signalling (Mohnen, 2008; Arsovski et al., 2009; Harholt et al., 2010; Kohorn and Kohorn, 2012).

The biosynthesis as well as post secretory modifications of pectin have been shown to be important in plant defence against pathogens. Arabidopsis mutants with defects in the biosynthesis of pectin such as a gae1 gae6 (glucoronate 4-epimerases) exhibit a compromised resistance to $B$. cinerea and Pseudomonas syringae infection (Bethke et al., 2016). The lines defective in the transcription factor AtEF014 with reduced pectin content in the cell walls have an increased susceptibility to $P$. syringae pv $D C 3000$ and enhanced resistance to $B$. cinerea (Zhang et al., 2016). Interestingly, some pectin enriched Arabidopsis mutants ( $p m r 5$ and pmr6) with enhanced resistance to powdery mildews (Erysiphe cichoracearum) have an enhanced susceptibility to $P$. syringae (Vogel, 2002; Vogel et al., 2004).

Proteins comprise only a small proportion of plant cell wall components, but they are an integral part as they contribute to structural integrity or modify cell wall composition during development and in response to environmental cues (Sommer-Knudsen et al., 1998; Fry, 2004; Passardi et al., 2004). The modifications of pectin such as acetylation and methylesterification (Liu et al., 2018) are known to play a role in cell wall integrity and resistance to plant pathogens. Arabidopsis rwa2 (reduced wall acetylation 2) mutants have a reduction in the acetylation of pectin, and are more resistant to B. cinerea (Manabe et al., 2011). Pectin methylestarase inhibitors (PMEI) are known to inhibit the activity of endogenous Arabidopsis pectin methylesterases (PMEs) which remove methylesters present on $\mathrm{HG}$ in either a random manner or blockwise fashion, exposing the galacturonic acid units (Willats et al., 2001). Arabidopsis plants overexpressing PMEI-1 and PMEI-2 showed greater resistance to the phytopathogens 
B. cinerea and Pectobacterium carotovorum (Lionetti et al., 2007). In Arabidopsis, four members of a berberine bridge enzyme like family were found to be responsible for oxidation of oligogalacturonides (OGs) derived from homogalacturonan hydrolysis. The oxidised OGs triggered weaker immune responses, but were more recalcitrant to hydrolysis by $B$. cinerea enzymes. Arabidopsis plants overexpressing these oxidases were shown to be more resistant to this pathogen (Benedetti et al., 2018). However, given the various modifications that occur in pectin, it remains to be fully elucidated how they impact plant pathogen interactions.

In this study, the potential role of cell wall-localised Arabidopsis protein BXL4 (BETAXYLOSIDASE 4) in the modification of RG-I and its impact on plant immunity is shown. Arabidopsis has a seven-member betaxylosidase (BXL) family, whose members possess glycosyl hydrolase domains whilst some have signal peptides for extracellular localisation (Goujon et al., 2003). The functional role of most of the members of this group are still to be determined but $B X L 1$ was shown to be a bifunctional $\beta$-Dxylosidase/a-L-arabinofuranosidase (Goujon et al., 2003; Minic, 2004), whose activity is important for extrusion of mucilage after hydration of Arabidopsis seeds (Arsovski et al., 2009). It has been hypothesised that $B X L 1$ trims arabinan side chains present in RG-I of both the primary cell wall and mucilage of seed coat epidermal cells. According to transcriptomics databases, BXL4 is upregulated upon infection by various pathogens and could therefore influence plant-pathogen interaction. Here, we provide evidence that $B X L 4$ acts on both xylose and arabinose in the cell wall. We can confirm that $B X L 4$ is induced by $B$. cinerea infection and mechanical wounding in a JA-lle dependent manner. We show that the bx/4 mutants have higher arabinose content but grow normally. The $b x / 4$ mutants are more susceptible when challenged with $B$. cinerea and display reduced levels of JA-lle and camalexin after infection.

\section{MATERIALS AND METHODS}

\section{Plant and Botrytis cinerea growth conditions}

Arabidopsis plants used for infection assays were grown on semi-sterile soil under short day conditions ( $8 \mathrm{~h}$ light and $16 \mathrm{~h}$ darkness) at a temperature of $22^{\circ} \mathrm{C}$ and a relative humidity of $65 \%$ in a Percival (Percival Scientific, Perry, USA). Arabidopsis plants for seed propagation were grown under long day conditions (16 h light and $8 \mathrm{~h}$ 
darkness), light intensity of $120-150 \mu \mathrm{mol} \mathrm{m}^{-2} \mathrm{~s}^{-1}$, at $22^{\circ} \mathrm{C}$ and $60 \%$ relative humidity in a climate chamber (York Industriekälte, Mannheim, Germany). The Arabidopsis mutants bxl1 (Ws ecotype; CS16299, Feldmann, 1991) and mpk3-DG (Li et al., 2002) and B. cinerea strain B05-10 (Staats and van Kan, 2012) were used for this work. The Botrytis spores were cultured on Potato dextrose broth (Sigma-Aldrich) plus agar, grown at RT for 10 days before harvesting by washing the spores off the plates using $1 / 4$ PDB and sieving through Miracloth to collect the conidoispores. Conidiospores were counted using a hemacytometer and stocks in $25 \%$ glycerol were made and stored at $-80^{\circ} \mathrm{C}$. T-DNA mutant lines of bx/4-1 (SALK_071629) and bx/4-2 (SAIL_331_B06) were sourced from Nottingham Arabidopsis Stock Centre and homozygous mutants where confirmed through genotyping PCR on genomic DNA using REDTaq ${ }^{\circledR}$ ReadyMix $^{\mathrm{TM}}$ (Sigma-Aldrich) following their protocol. Primers used are listed in table S1.

Gene expression analysis ( $q R T-P C R$ )

RNA was extracted from leaves using Spectrum ${ }^{\text {TM }}$ Plant Total RNA Kit (SigmaAldrich). cDNA from DNasel (Thermo Scientific, Waltham, MA, USA) treated RNA was made using Revert Aid $^{\mathrm{TM}} \mathrm{H}$ minus Reverse Transcriptase (Thermo Scientific). cDNA derived from leaf RNA was used for qRT-PCR using Takyon ${ }^{\mathrm{TM}}$ No Rox SYBR ${ }^{8}$ MasterMix dTTP Blue (Eurogentec, Lüttich, Belgium). Quantification of expression was done relative to ACTIN8. The primers used are found in table $\mathrm{S} 1$.

\section{Molecular cloning and Arabidopsis transformation}

The R4 Gateway Binary Vectors (R4pGWB; Nakagawa et al., 2008) were employed to make the constructs used in this work. The TBA2 constructs with and without a citrine tag were made by first amplifying the TBA2 promoters using PCR and cloning into entry vector pDONRP4-P1R. The cDNA constructs were made by amplifying the cDNA with PCR (primers used in Table S1) and cloning into entry vector pDONR207. A tripartite LR reaction was performed to incorporate the TBA2 promoter and CDNA into R4pGWB501 (modified vector) with and without citrine tag (Nakagawa et al., 2008). Arabidopsis plants were transformed by floral dipping as described (Clough and Bent, 1998) and the T1 seeds grown on MS medium supplemented with hygromycin for selection. 


\section{Confocal microscopy}

The transformed Arabidopsis T2 seeds were visualised under a confocal microscope for localisation of BXL proteins. Confocal images were recorded using confocal microscope Zeiss LSM 780 (Carl Zeiss Inc., Jena, Germany). Citrine was excited at $488 \mathrm{~nm}$ through a $488 \mathrm{~nm}$ major beam splitter (MBS). Detection of fluorophore was done at a wavelength of $514-530 \mathrm{~nm}$.

\section{Botrytis cinerea infection assay}

B. cinerea spores were diluted to $5 \times 10^{5}$ spores per millilitre in $1 / 4$ Potato dextrose broth (Sigma-Aldrich) for drop infection assay or Vogels (Vogel, 1956) media for spray infection assay used for qRT-PCR analysis. The spores were pre-germinated for $4 \mathrm{~h}$ before infections were carried out. For drop infections, $6 \mu \mathrm{L}$ of spore suspension in $1 / 4$ PDB was carefully placed on the adaxial side (away from the midrib) of a fully expanded rosette leaf of 6-7 weeks old Arabidopsis (at least 30 leaves were used from 10 independent plants). For spray infection, plants were sprayed until spray droplets began to run off the leaves. Plants were then covered and grown under high humidity conditions for $72 \mathrm{~h}$. Lesion diameters were measured using a veneer calliper. For spray infections, whole rosettes were harvested at day zero ( $\mathrm{d} 0$ immediately after spraying) and at $72 \mathrm{~h}$ post infection (d3). For fungal genome quantification, fungal DNA was extracted using Plant/Fungi DNA isolation Kit (Norgen Biotek Corp) following manufacturer's protocol. The fungal $\beta$-ACTIN genomic DNA was quantified by qPCR (Ettenauer et al., 2014) and primers used are listed in Table S1.

\section{Mucilage staining with ruthenium red}

$5 \mathrm{mg}$ of Arabidopsis seeds were placed in $500 \mu \mathrm{L} \mathrm{dd}_{2} \mathrm{O}$ in an Eppendorf tube before being gently shaken for $1 \mathrm{~h}$ on a rotary shaker. Water was gently removed and $500 \mu \mathrm{L}$ $0.02 \%$ ruthenium red was added. Seeds were put back on the shaker for another 15 min before the ruthenium red was removed and seeds resuspended in $500 \mu \mathrm{L} \mathrm{dd} \mathrm{H}_{2} \mathrm{O}$ added again. A droplet with stained seeds was placed on a microscopic slide and viewed under a light microscope. 
Mucilage was extracted by agitating $5 \mathrm{mg}$ of seeds in water for two $\mathrm{h}$ on a rotary shaker. Seeds were then allowed to settle for a few minutes before $1 \mathrm{~mL}$ mucilage solution was removed and placed in a Duran culture tube. Mucilage solution was evaporated in a water bath at $40^{\circ} \mathrm{C}$ under nitrogen stream. Dry samples were hydrolysed for $1 \mathrm{~h}$ at $121^{\circ} \mathrm{C}$ using $2 \mathrm{M}$ trifluoroacetic acid, before being evaporated again. $100 \mu \mathrm{L}$ allo-inositol and $500 \mu \mathrm{L} \mathrm{dd}_{2} \mathrm{O}$ were added to resuspend the hydrolysed mucilage. $20 \mu \mathrm{L}$ sample was evaporated under a nitrogen stream before overnight derivatisation in $15 \mu \mathrm{L}$ methoxyamide (MOX, $30 \mathrm{mg} / \mathrm{mL}$ in anhydrous pyridine). The next day, $30 \mu \mathrm{L}$ MSTFA (N-Methyl-N-(trimethylsilyl)trifluoroacetamide) were added and samples were analysed GC-MS 1-6 $\mathrm{h}$ after MSTFA addition.

\section{GC-MS analysis}

Samples were analysed with a 7890B GC-System coupled to a 5977B MSD quadrupole set-up from Agilent Technologies. GC-separation was achieved on a HP5 column (Agilent Technologies) using the following temperature gradient: $150^{\circ} \mathrm{C}$ for $2 \mathrm{~min}, 5 \mathrm{~K} / \mathrm{min}$ gradient for $20 \mathrm{~min}, 15 \mathrm{~K} / \mathrm{min}$ to a final temperature of $320^{\circ} \mathrm{C}$, which was held for $3 \mathrm{~min}$. For each run, $1 \mu \mathrm{L}$ of the derivatised sample was injected. Identification of compounds was done by a combination of retention times compared to external standards and MS-spectra. Prepared mucilage samples were quantified relative to the internal standard allo-inositol. In parallel runs, monosaccharide standards of different concentration were used to determine response factors for areato-molar amount conversion allowing absolute quantification.

\section{Monsaccharide analysis of the alcohol insoluble residue of leaves}

The alcohol insoluble residue (AIR) was extracted from plant leaves (6-7 weeks old plants) grown in the dark for $48 \mathrm{~h}$. The AIR was extracted as described (Gille et al., 2009). The leaves were flash frozen in liquid nitrogen before they were pulverised using mortar and pestle. The ground material was washed 2 times with $70 \%(\mathrm{v} / \mathrm{v})$ ethanol, washed thrice with a chloroform:ethanol mixture $(1: 1[\mathrm{v} / \mathrm{v}])$, and lastly with acetone, before being air dried. Hot water pectin extraction (Yeoh et al., 2008) was used by shaking $2 \mathrm{mg}$ of $\mathrm{AIR}$ in $1.4 \mathrm{~mL} \mathrm{dd}_{2} \mathrm{O}$ at $90^{\circ} \mathrm{C}$ for $2 \mathrm{~h}$. Monosaccharide analysis was then carried out on AIR using the same GC-MS method used on mucilage as described above. 


\section{Phytohormone measurements}

Extraction of phytohormones was carried out as described by Kusch et al., 2019. Reversed phase separation of constituents was performed as previously described (Kusch et al. 2019) using an ACQUITY UPLC® system (Waters Corp., Milford, MA, USA) equipped with an ACQUITY UPLC® HSS T3 column (100 mm x 1 mm, $1.8 \mu \mathrm{m}$; Waters Corp., Milford, MA, USA). Nanoelectrospray (nanoESI) analysis was carried out as described in Kusch et al., (2019) and phytohormones were ionized in a negative mode and determined in a scheduled multiple reaction monitoring mode with an $A B$ Sciex 4000 QTRAP® tandem mass spectrometer (AB Sciex, Framingham, MA, USA). Mass transitions were used as described by Iven et al., (2012), with some modifications specified in Table S2.

\section{RESULTS}

$B X L 4$ localises to the apoplast

The BXL4 protein possesses a predicted signal peptide for secretion to the apoplast (Goujon et al., 2003). To test if the protein has cell wall localisation, a construct of $B X L 4$ with a C-terminal Citrine fusion ( $B X L 4$-Citrine) driven by the seed coat specific TBA2 (TESTA ABUNDANT2) promoter (Tsai et al., 2017) was generated and transformed into Arabidopsis bx/1 mutant and Col-0 to generate stable expression lines. Arabidopsis seed coat epidermal cells of T2 seeds expressing pTBA2:BXL4CITRINE were visualised under a confocal microscope $7 \mathrm{~d}$ post anthesis. The BXL4CITRINE fluorescence could be strongly detected in the mucilage pocket of seed coat epidermal cells (Figure 1A and $1 \mathrm{~B}$ ) similar to the control line $\mathrm{pTBA2:BXL1-CITRINE}$ (Supplementary Figure1).

\section{$B X L 4$ rescues the mucilage phenotype of bx/1}

While in the Arabidopsis wild type (ecotype Wassilevskia, Ws), mucilage is extruded from the seed coat, the $b x / 1$ knockout mutant has a defect in mucilage extrusion after hydration of the seeds (Figure 2A) (Arsovski et al., 2009). To test if the putative betaxylosidase BXL4 could perform a similar enzymatic activity and therefore rescue the mucilage defect phenotype, the $b x / 1$ knockout mutant was complemented with the pTBA2: BXL4 construct with and without a Citrine tag. The T2 seeds were placed in 
water and the mucilage was stained with ruthenium red. The results indicated that the bx/1 mutant line could be complemented with pTBA2:AtBXL4 (Figure 2C) with and without the Citrine tag, in a similar way as with pTBA2: BXL1 (Figure 2D), as these lines could extrude their mucilage in the same manner as wild type Ws.
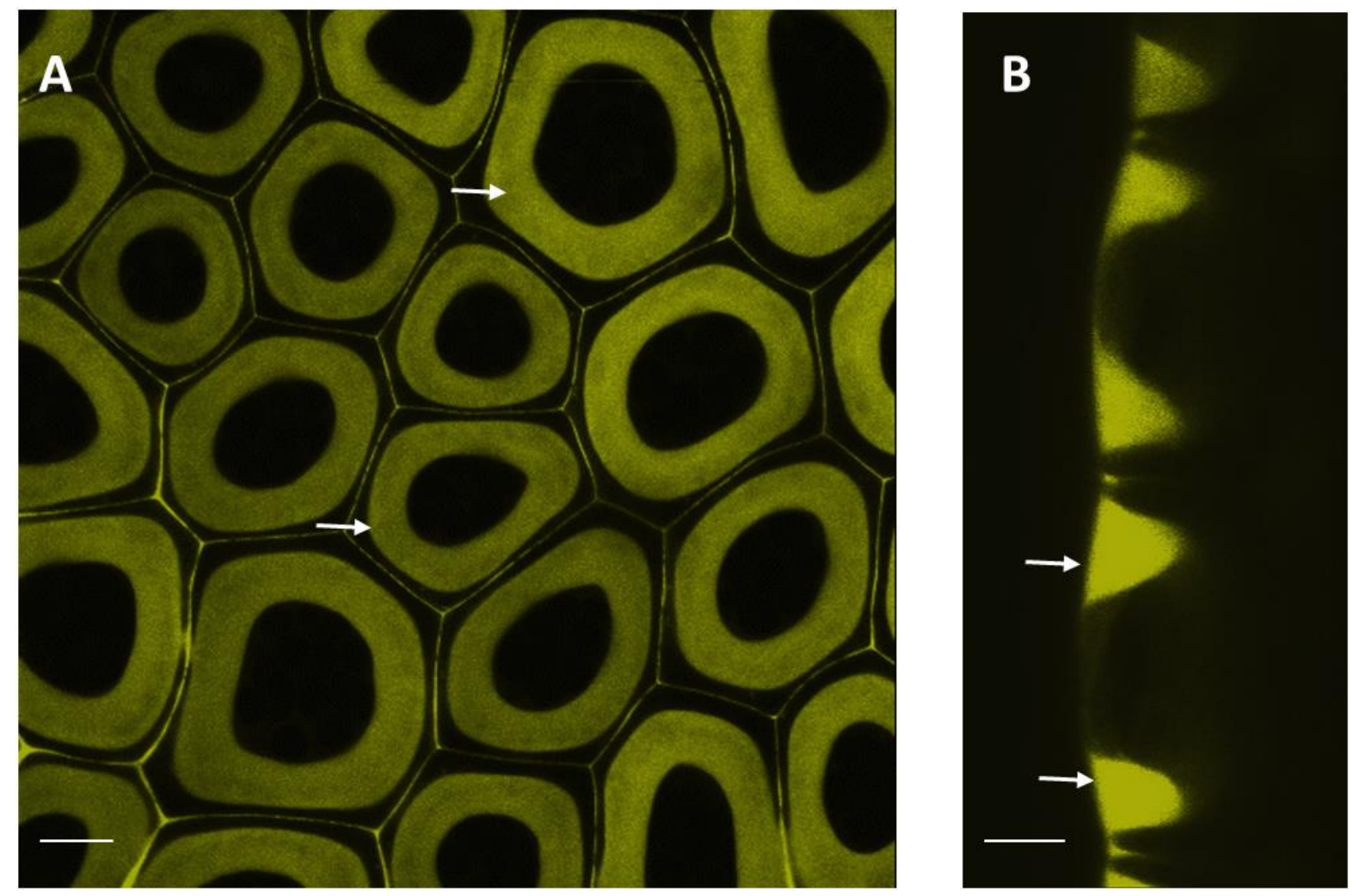

Figure 1: BXL4-CITRINE localises to the apoplast in Arabidopsis seed coat epidermal cells

(A) BXL4-CITRINE stably expressed under the TBA2 promoter localises to the apoplast (deposited predominantly in the mucilage pocket) of Arabidopsis seed coat epidermal cells 7 days post anthesis (dpa). Single plane images of the seed coats were obtained by confocal microscopy. The apopolast of the of seed coat epidermal cells appears like a doughnut ring that surrounds the cytoplasm when imaged from the top. (B) Visualisation of the seed coat epidermal cells from the side at 7 dpa shows the apoplast as two pockets in the apical corners of the cells (arrow). Scale bars, $10 \mu \mathrm{m}$

The Arabidopsis $b x / 1$ knockout mutant is known to produce mucilage pectin with higher levels of arabinans (Arsovski et al., 2009). To investigate if the pTBA2:BXL4 complemented $b x / 1$ lines had mucilage with a monosaccharide composition similar to wild type Ws, analysis was done on the water extracted mucilage from the T2 seeds. Extracted mucilage was hydrolysed with TFA before derivatisation with MOX and 
MSTFA to enable monosaccharide composition analysis by GC-MS. Mucilage is composed mainly of pectin RG-I (Dean et al., 2007; Arsovski et al., 2009; Arsovski et al, 2010; Haughn \& Western, 2012), and consistent to that, monosaccharide analysis showed that rhamnose and galacturonic acid were the most abundant sugars. The bx/1 mutant exhibited a 4-fold increase in abundance of arabinans in comparison to the wild type Ws, while the $b x / 1$ lines complemented with pTBA2:BXL1 and pTBA2:BXL4 showed arabinose levels reverted to wild type levels (Figure 2E). The

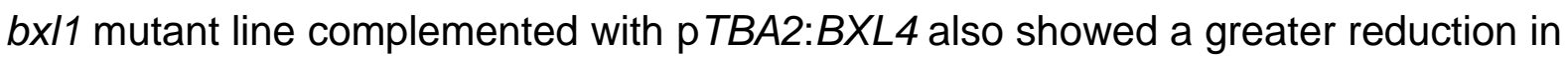
xylose compared to the wild type (Figure $2 \mathrm{E}$ ). Arabidopsis lines overexpressing $B X L 4$

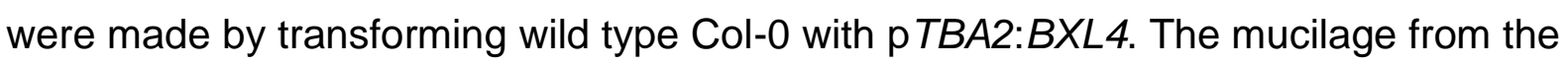
T2 seeds was extracted and analysed for monosaccharide composition analysis using GC-MS. The overexpression lines showed arabinose levels similar to wild type, whilst the xylose composition was further depleted (Figure 2F).

\section{Disruption of BXL4 does not affect plant growth}

Alteration of the cell wall composition may result in the disruption of normal growth and development of Arabidopsis plants (Noguchi et al., 1997). To check if knocking out $B X L 4$ affects the normal growth of Arabidopsis, two Arabidopsis T-DNA insertion lines $b x / 4-1$ and $b x / 4-2$ that carry insertions in exon 4 and 5 (Figure $3 A$ ) were obtained from the stock centre. To confirm the position of the insert, the genomic DNA of the mutants was sequenced. To check if the two lines were knockouts, the expression of $B X L 4$ in the T-DNA insertion lines by quantitative reverse transcription polymerase chain reaction (qRT-PCR) was done on RNA extracted from 4 weeks old plants grown on soil. The $b x / 4-1$ line appears to be a knockout line whilst $b x / 4-2$ showed a small amount of transcript (Figure 3B). Gene expression was normalised to reference gene ACTIN8 and relative to Col-0. To check if knocking out of BXL4 had effects on the monosaccharide composition of the pectin, water extracted pectin from alcohol insoluble residues (Gille et al., 2009) of leaf material from 6 weeks old Arabidopsis plants was analysed for monosaccharide composition using GC-MS. The monosaccharides were normalised to rhamnose. The bx/4 mutants showed an increased abundance of arabinose compared to Col-0. There was no significant difference in the xylose content (Figure $3 \mathrm{C}$ ) or any other pectin monosaccharide 
measured. Arabidopsis bx/4 mutants did not exhibit any obvious growth defects and were comparable to Col-0 (Figure 3D).
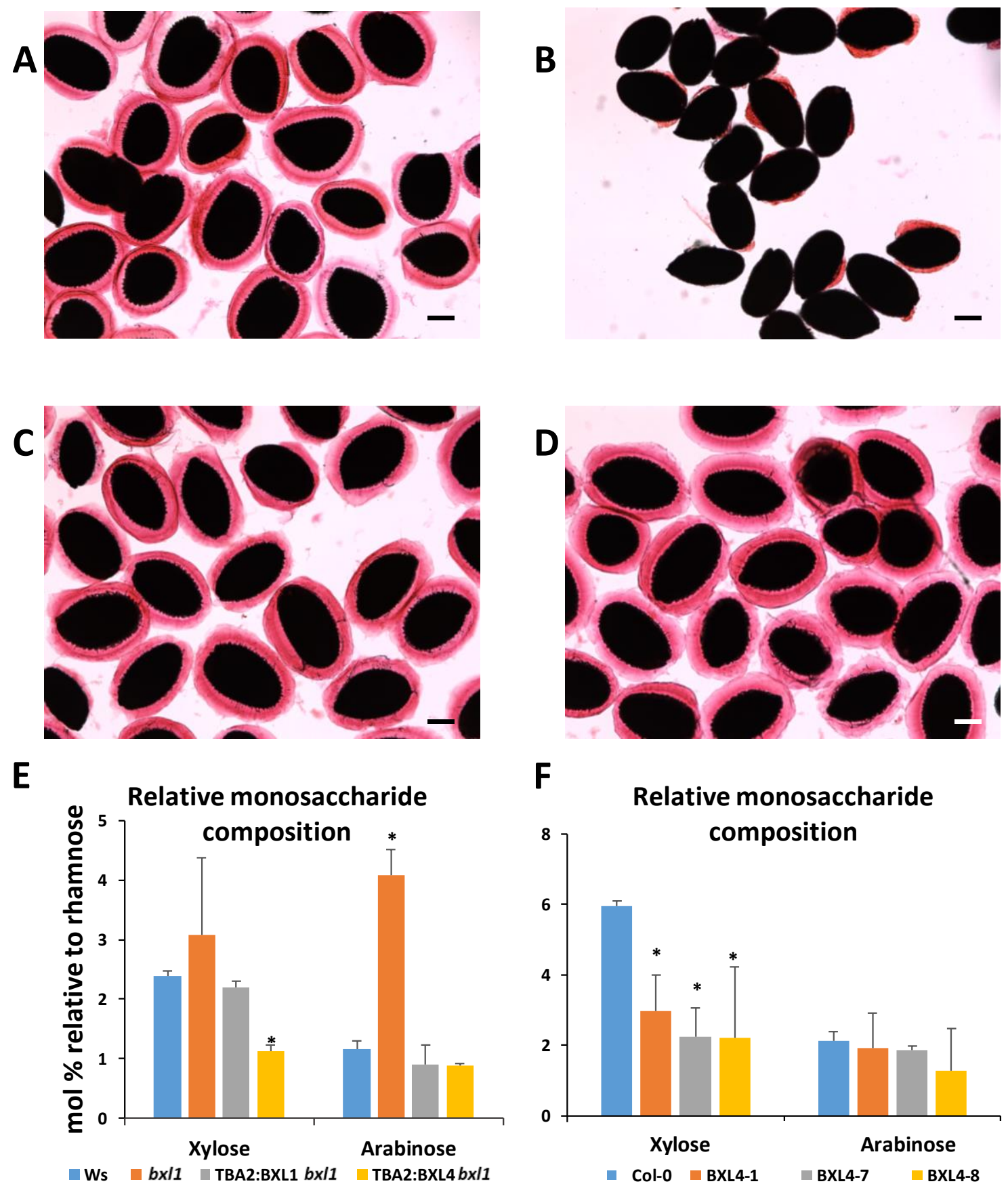

Figure 2: BXL4 can complement the mucilage phenotype of bxl1.

(A) In the wild type (ecotype Ws), the extrusion of the ruthenium red-stained mucilage forms a halo around the seeds. (B) $b x / 1$ seeds extrude their mucilage in a patchy manner. (C-D)This phenotype can be complemented by introducing pTBA2:BXL1 (C) or pTBA2:BXL4 (D) into the bxl1 background. Bars, $100 \mu \mathrm{m}$. (E) Relative monosaccharide composition of mucilage extracted from Ws, bxl1, bx/1 pTBA2:BXL1 and bxl1 p pTBA2:BXL4. (F) Monosaccharide composition of mucilage extracted from wild type Col-0 and three BXL4 overexpression lines (pTBA2:BXL4 line 1, 7 and 8). Monosaccharide composition was determined by GC-MS and 
normalised to rhamnose. Error bars show SD ( $n=3$ biological replicates), Statistical difference to WT (Student's t-test ), ${ }^{*}$ indicates $p<0.05$.

A

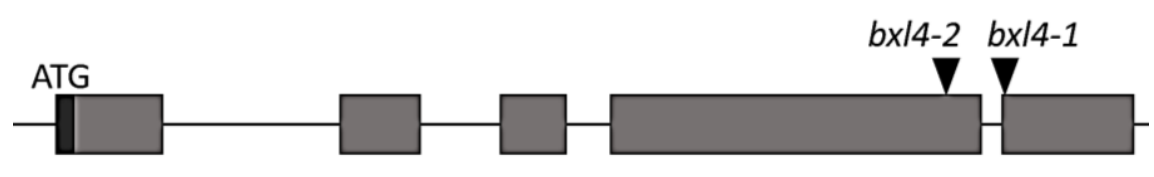

B

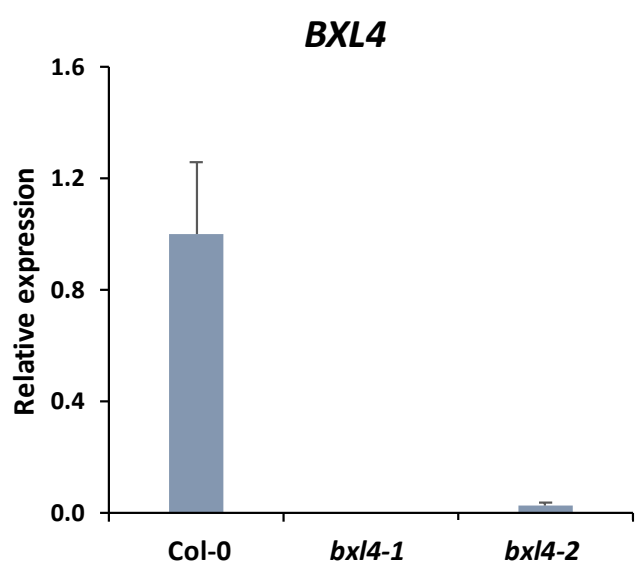

C Relative monosaccharide

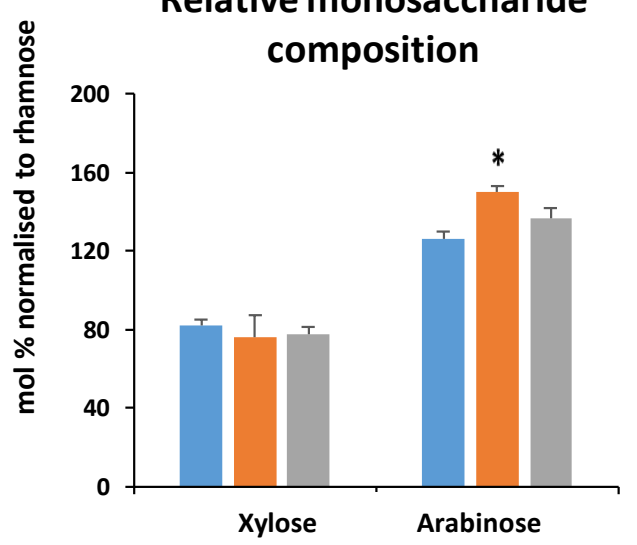

D

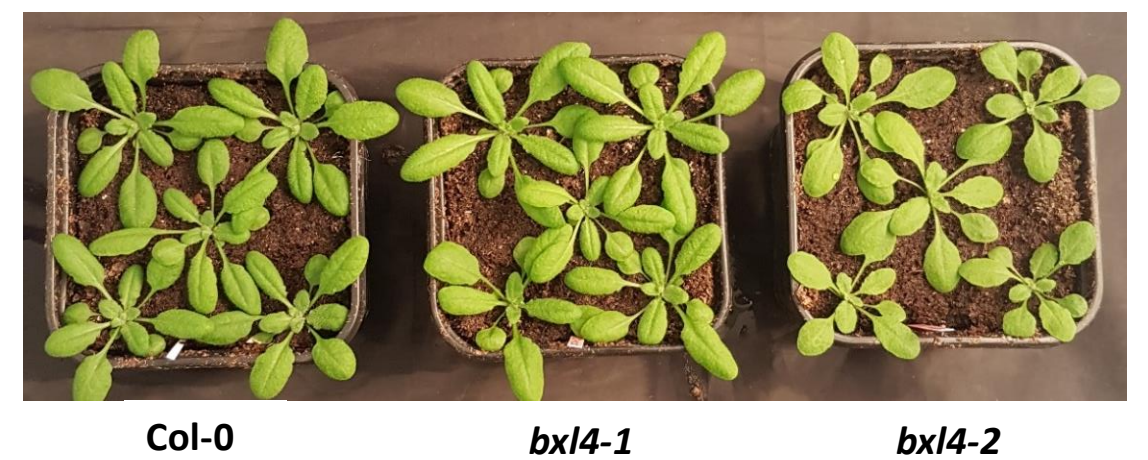

Figure 3: The disruption of BXL4 has mild effects on the cell wall composition but does not alter plant growth.

(A) The intron-exon structure of BXL4 and the T-DNA insertion positions of $b x / 4-1$ and $b x / 4-1$. The exons are represented by grey boxes, introns black lines, black triangles show positions of the T-DNA insertions and the UTR black box. (B) Relative expression of BXL4 from RNA extracted from leaves of WT and bx/4 mutant lines determined by qRT-PCR using primers shown in Supplementary table 1. Expression values were normalised to the reference gene ACTIN 8 and are presented relative to the wild type levels. Error bars represent standard error of three biological replicates. (C) Monosaccharide composition of water extracted pectin from Arabidopsis leaves of wild type, $b x / 4-1$ and $b x / 4-2$ mutant lines. The monosaccharides were normalised to rhamnose. Water extracted pectin was analysed by GC-MS. Error bars represent SD ( $n=4$ biological replicates), Statistical difference to WT (Student's t-test), ${ }^{*} \mathrm{P}<0.05$. 
BXL4 expression is wound and Botrytis infection induced and jasmonoyl-isoleucinedependent

According to publicly available databases, $B X L 4$ is upregulated by infection with various pathogens. To confirm that $B X L 4$ is a stress-induced gene, its expression pattern after mechanical wounding and infection with $B$. cinerea was analysed. Generally, BXL4 gene expression is relatively low in Col-0 grown under normal conditions. However, the gene was induced 15-fold upon wounding of the rosettes (Fig. 4A). Induction of BXL4 was also investigated in the jasmonoyl-isoleucine (JA-lle) deficient mutant line dde2-2 (von Malek et al., 2002), because JA-lle regulates the expression of many wounding responsive genes (Turner et al., 2002). Relative to wild type expression, upregulation of BXL4 transcript after wounding was greatly reduced in the dde2-2 mutant (Figure $4 A$ ). To confirm that $B X L 4$ is a $B$. cinerea infection associated gene, accumulation of $B X L 4$ transcript after infection with $B$. cinerea was checked and showed significant induction in Arabidopsis after infection. Mechanical wounding and attack from necrotrophic pathogens in Arabidopsis is known to trigger defence responses regulated via JA-lle signalling (Turner et al., 2002). To check if the bx/4 mutants had impaired JA-lle signalling, the transcript abundance of JA-lle reporter genes JAZ10 (Yan et al., 2007; Chung et al., 2008) and PDF1.2 (Penninckx, 1998; Zarei et al., 2011) after wounding was tested in wounded Col-0 and $b x / 4$ mutants. The bx/4 mutants had a decreased transcript accumulation of JAZ10 (Figure 4B) and PDF1.2 (Figure 4C) at $2 \mathrm{~h}$ post wounding compared to Col-0. Furthermore, the transcript accumulation of the JA-lle associated marker genes JAZ10 and PDF1.2 after necrotrophic pathogen infection was also assessed. Transcript accumulation of JAZ10 did not show a significant difference between the wild type and the $b x / 4$ mutants (Figure 4E) whilst PDF1.2 was higher in Col-0 compared to bx/4 mutants and expression increased with increased disease development (Figure 4F). Finally, the relative expression of $P A D 3$, a gene involved in camalexin biosynthesis that is induced after attack from necrotrophic pathogens (Zhou et al., 1999), was measured in Col-0 and bx/4 mutant lines after infection with $B$. cinerea. $P A D 3$ and camalexin are known to play a defensive role upon infection of Arabidopsis by both biotrophic and necrotrophic pathogens (Ferrari, 2003; Schuhegger et al., 2006). The transcript accumulation of $P A D 3$ in RNA extracted from $b x / 4$ knockout mutant lines after infection 
was reduced compared to Col-0. Expression of PAD3 was normalised to ACTIN8 and is shown relative to Col- 0 at $0 \mathrm{~h}$ (Figure 4G).
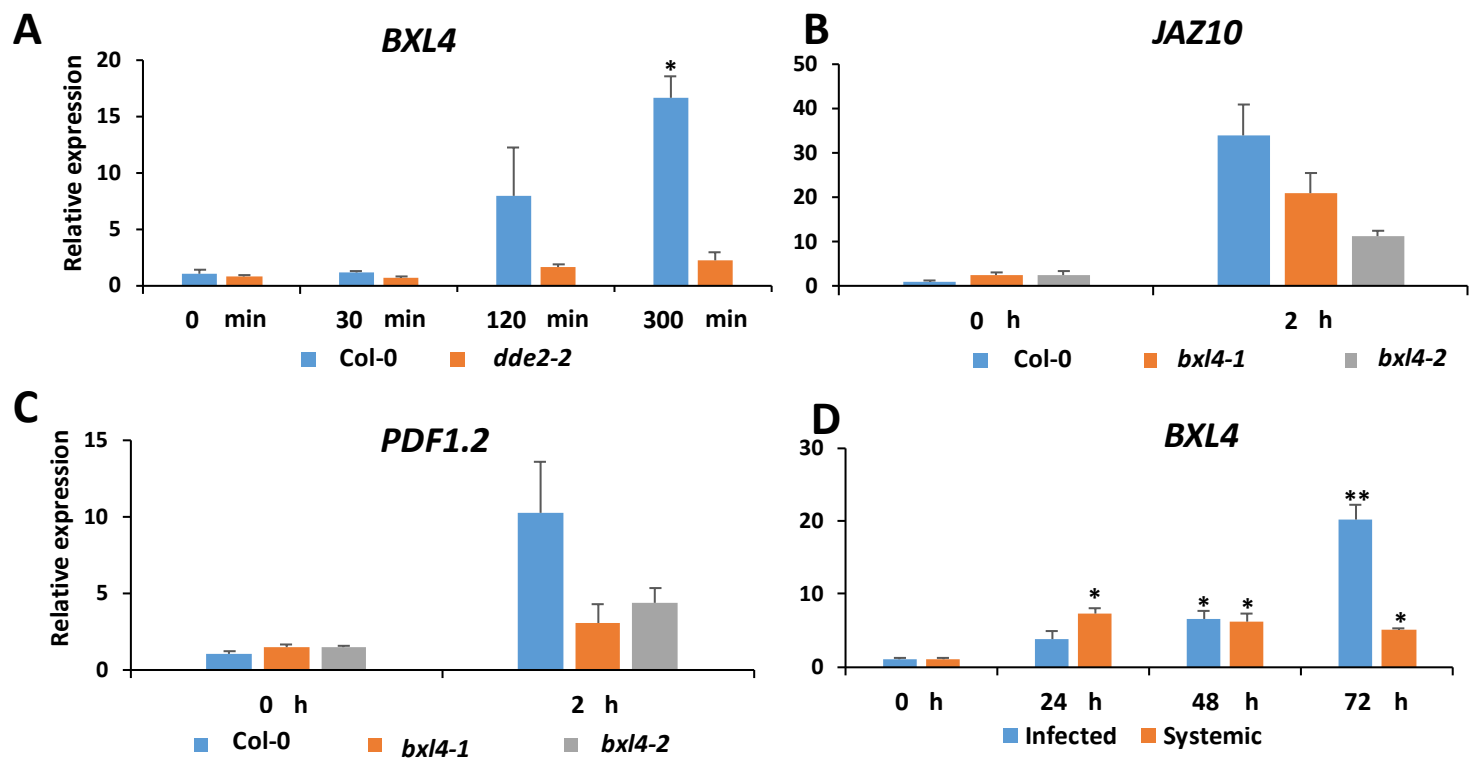

E
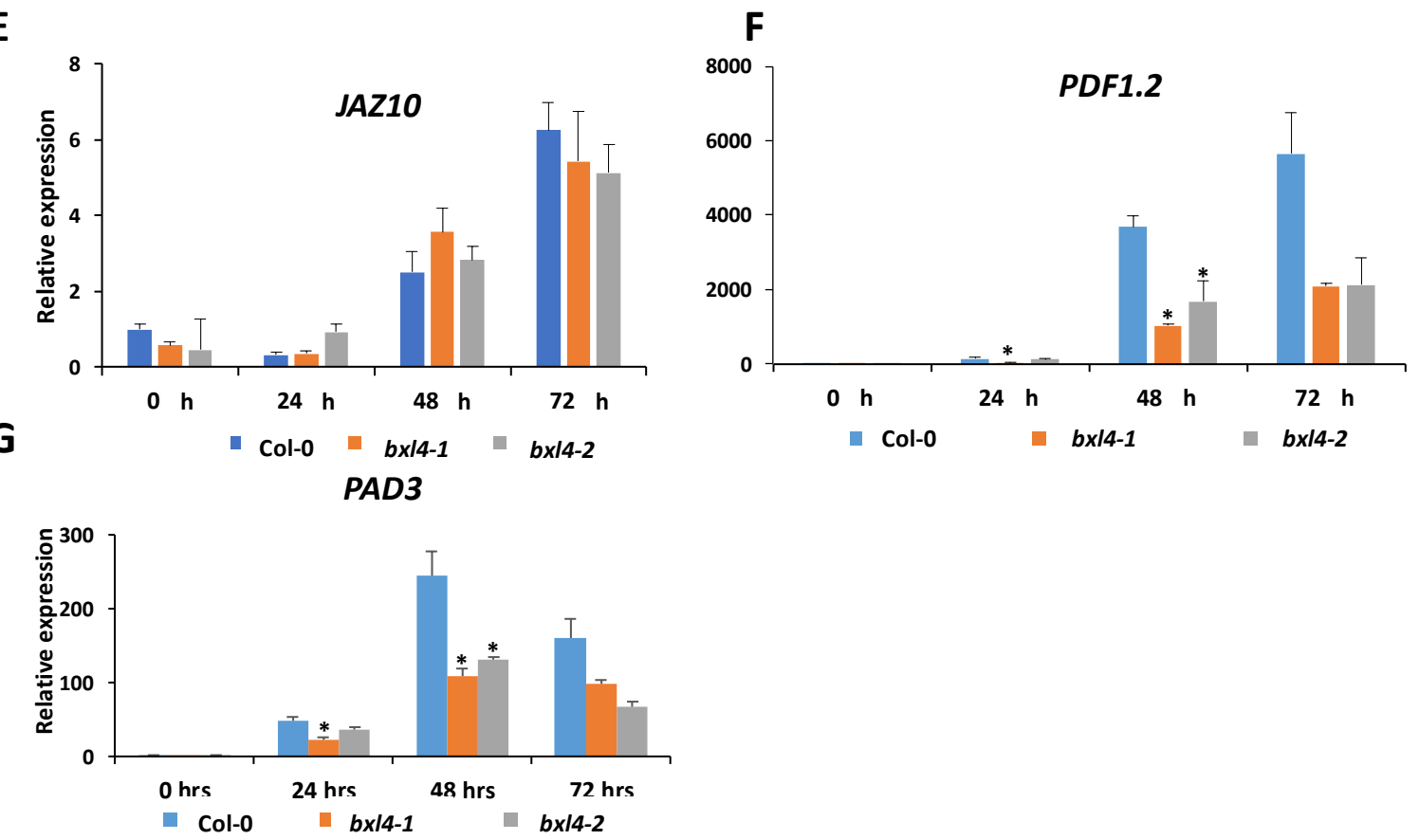

Figure 4: Expression of BXL4 is induced by wounding and $B$. infection and acts upstream of jasmonoyl-isoleucine signalling.

(A) Expression pattern of $B X L 4$ in Col-0 and jasmonoyl-isoleucine (JA-lle)-deficient mutant dde2-2. RNA for qRT-PCR was extracted from 6 weeks old plants harvested before wounding (0 mins) and at 30, 120 and 300 min after wounding. (B-C) Relative expression of JA-lle marker genes JAZ10 (B) and PDF1.2 (C) in Col-0, bx/4-1, and bx/4-2 at 0 and 2 hours post wounding. (D) Relative expression of $B X L 4$ in 6 week old Col-0 plants drop inoculated with $B$. cinerea conidiospores. Samples were collected from infected and systemic leaves at 0 (before 
infection), 24, 48 and $72 \mathrm{~h}$ after infection. (E-F) Relative expression of JA-lle marker gene JAZ10 (E) and PDF1.2 (F) in Col-0, bx/4-1 and bx/4-2 at 0, 24, 48 and $72 \mathrm{~h}$ after infection with B. cinerea. Relative expression was measured by qRT-PCR, normalised to reference gene ACTIN8, and relative to wild type 0 . Error bars represent standard error of 3 biological replicates. Statistical difference to WT (Student's t-test), ${ }^{*} P<0.05,{ }^{*} P<0.01$. (G) Relative expression of camalexin marker gene $P A D 3$ in $B$. cinerea infected Col-0 and bx/4 mutant lines. RNA extracted from samples collected at $0,24,48,72 \mathrm{~h}$ after infection and normalised to ACTIN8, relative to Col-0 at $0 \mathrm{hrs}$. Error bars show standard error of 3 biological replicates.

bx/4 mutant plants have a reduced production of JA and camalexin upon infection with B. cinerea

To confirm if the observed reduction in expression of PDF1.2 and PAD3 in bx/4 mutants correlated with a reduction in accumulation of JA and camalexin respectively, the levels of the JA and camalexin which are triggered by infection with $B$. cinerea (Nie et al., 2017; Ferrari et al., 2003, 2007; Scalschi et al., 2015) were measured. The bx/4 mutants showed a reduction in JA production at $72 \mathrm{~h}$ post infection compared to Col0 (Figure 5A). There was also a reduction in the accumulation of the JA active derivative JA-lle ( Thines et al., 2007; Yan et al., 2009) in the bx/4 mutants at $72 \mathrm{~h}$ post infection (Figure 5B). The abundance of camalexin after $B$. cinerea infection was reduced at $72 \mathrm{~h}$ post infection in the bx/4 mutants compared to Col-0 (Figure 5C). The wounded bx/4 mutants also had a slightly reduced accumulation of JA and JA-Ile compared to Col-0 (Supplementary Figure 3).

\section{$B X L 4$ contributes to resistance against $B$. cinerea}

After confirming that $B X L 4$ is a stress induced gene, we went on to check if the $b x / 4$ mutants had a compromised resistance to $B$. cinerea, and the plants were drop inoculated with $5 \times 10^{5}$ conidiospores $/ \mathrm{mL}$ and lesion diameter measured $72 \mathrm{~h}$ post inoculation. The $b x / 4$ mutants developed significantly larger lesions compared to Col-0. mpk3 was used as a susceptible control (Galletti et al., 2011) (Figure 6A \& 6C). An additional infection method was used to confirm susceptibility to $B$. cinerea, which involved spraying the plants and quantifying the fungal $\beta$ ACTIN genomic DNA (Ettenauer et al., 2014) using qPCR. Fungal DNA was quantified immediately after spraying and after 3 days. The bx/4 mutants showed a significantly higher accumulation of fungal genomic DNA as compared to Col-0 (Figure 6B). 
A

$J A$

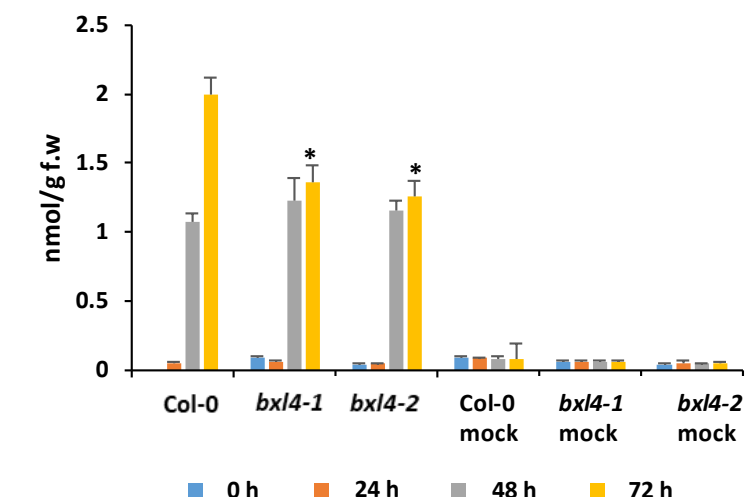

C

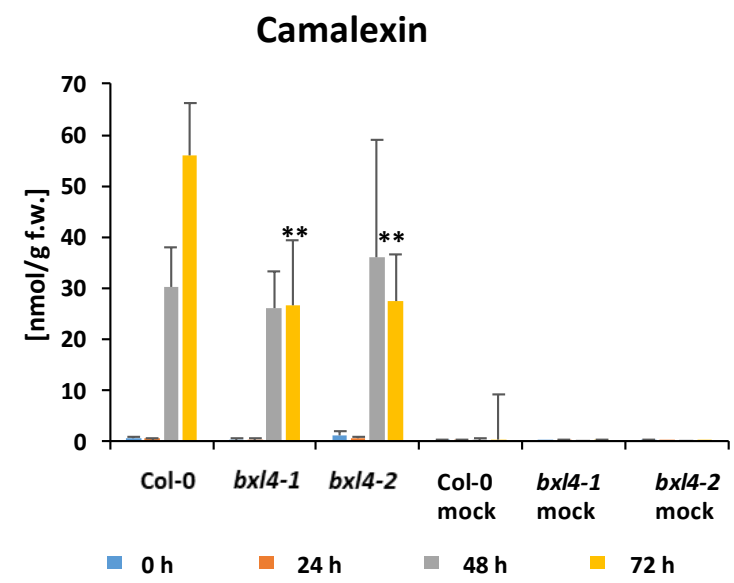

B

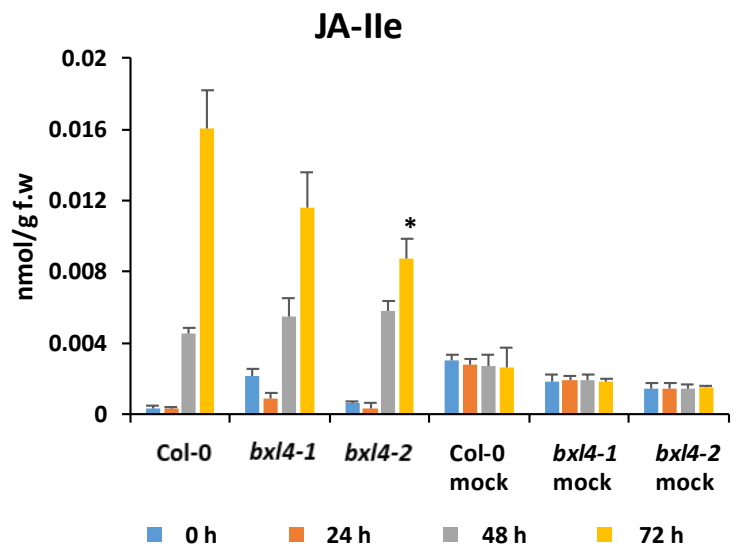

Figure 5: Levels of $B$. cinerea infection-induced JA, JA-lle and camalexin are reduced in bx/4 mutant lines

Col-0 and $b \times 14$ mutant lines were spray-infected with $B$. cinerea and leaves were sampled at 0, 24, 48 and $72 \mathrm{~h}$ after infection. Extracted levels of JA (A), JA-Ile (B) and camalexin (C) were analysed using nanoESI. Error bars represent standard deviation of 6 biological replicates, statistical difference to WT (Student's t-test ), ${ }^{*} \mathrm{P}<0.05$, ${ }^{*} \mathrm{P}<0.01$.

\section{DISCUSSION}

The importance of pectin biosynthesis and modification in plant defences has been elucidated in many previous publications. Here, we provide evidence that BXL4 modifies cell wall polysaccharides contributing to immunity against $B$. cinerea.

$B X L 4$ acts on arabinans present in the apoplast

Our BXL4-Citrine fusion protein driven by a seed coat specific promoter TBA2, localised in the mucilage pocket of Arabidopsis seed coat epidermal cells at seven 
A

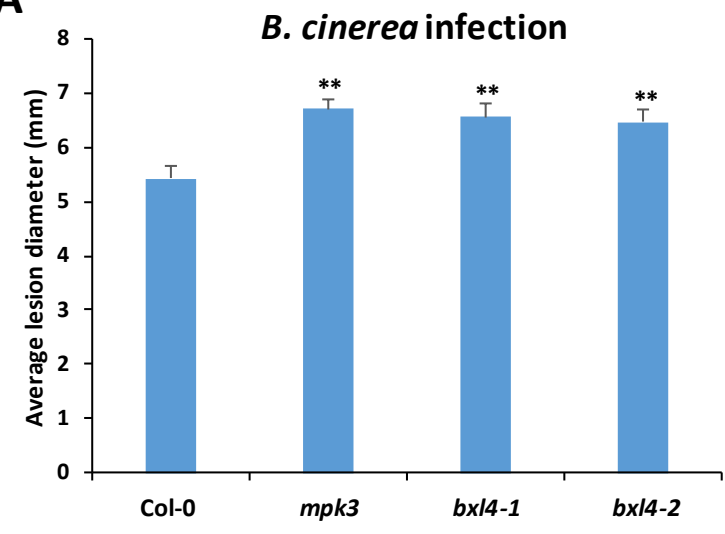

B

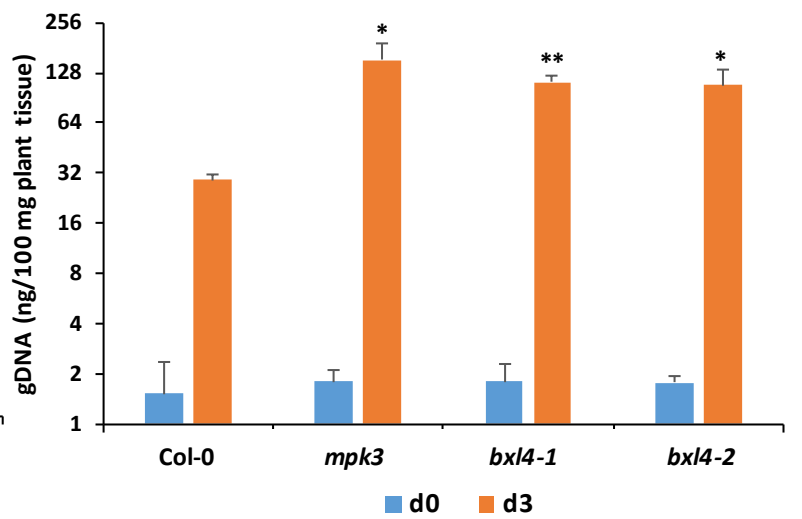

\section{C}

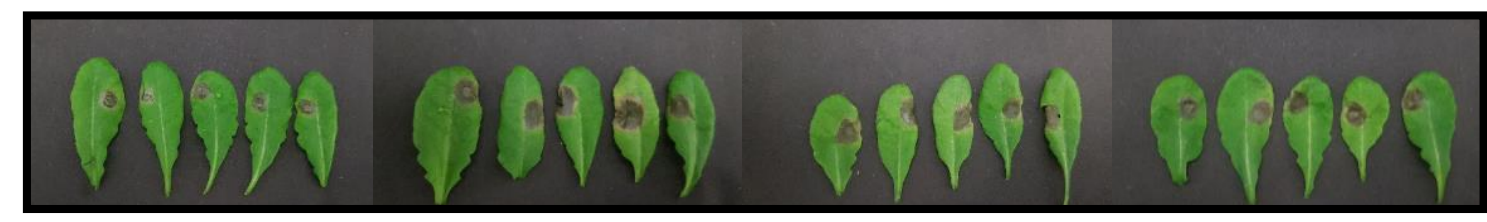

Col-0

mpk3

bx/4-1

bx14-2

Figure 6: bxl4 mutant lines are more susceptible to $B$. cinerea infection

(A) Infection phenotype of Col-0, bx/4-1 and bx/4-2 after $B$. cinerea infection. A minimum of 30 leaves from 5 independent plants were drop inoculated with $6 \mu \mathrm{L} B$. cinerea conidiopores $\left(5 \times 10^{5}\right.$ spores $\left./ \mathrm{mL}\right)$, and lesion diameter measured 3 days after infection. mpk3 was used as the susceptible control. Error bars show SE, Statistical difference to WT (Student's t-test), ${ }^{* *} P<0.01$. (B) Infection phenotype measured after spraying plants with $B$. cinerea conidiospores and quantifying fungal genomic DNA with qPCR. Fungal genomic DNA was quantified immediately after infection (d0) and 3 days after infection (d3). Error bars show SE ( $n=3$ biological replicates), Statistical difference to WT (Student's t-test), ${ }^{*} P<0.05,{ }^{* *} P<0.01$. (C) Lesion phenotype of detached leaves 3 days post infection with $B$. cinerea.

days post anthesis. The apoplastic localisation was consistent with work by Goujon et al., (2003) and data obtained using SignalP-5.0, which showed that BXL4 has a predicted signal peptide for localisation in the extracellular matrix. It is known that mucilage and RG-I modifying proteins, including BXL1 are deposited in the mucilage pocket between four and eight days post anthesis (Western et al., 2000; Arsovski et al., 2009; Haughn and Western, 2012; Breitenbach et al., 2014). Our observation of $B X L 4$ localisation in the apoplast means it is in the right compartment to perform cell wall remodelling.

The Arabidopsis bx/1 knockout mutant shows a distinct phenotype as the mucilage from hydrated seeds extrudes in a delayed and uneven manner (Arsovski et al., 2009). 
To check if $B X L 4$ was functionally similar to its homologue $B X L 1$, we expressed pTBA2:BXL4 in the mucilage of the bx/1 mutant. Examination of the T2 seeds of pTBA2:BXL4 bx/1 lines revealed that they extruded mucilage normally. $B X L 4$ has a predicted glycosyl hydrolase domain (Goujon et al., 2003), and thus should be capable of carrying out similar enzymatic functions as $B X L 1$. The TBA2 protein is one of the most abundant and highly expressed seed coat proteins (Tsai et al., 2017). Therefore, the TBA2 promoter was used for ectopic expression in the seed coat upon which rescue of the mucilage phenotype could be observed for $B X L 4$.

$B X L 1$ is a known bifunctional $\beta-D-x y l o s i d a s e / \alpha-L$-arabinofuranosidase and the $b x / 1$ knockout mutant has an abundance of (1-5)-linked arabinans which can form one of the side chain present in mucilage RG-I (Arsovski et al., 2009). Expression of pTBA2:BXL4 in the bx/1 mutant background resulted in the production of mucilage with a reduced arabinose content similar to wild type. The arabinosidase activity of BXL4 was also suggested by the increased abundance of arabinans in the leaf cell walls of bx/4 mutants. The generation of $B X L 4$ overexpression lines under the TBA2 promoter in wild type Col-0 showed a further decrease in the xylose content compared to Col-0, whilst the arabinan levels were comparable to Col-0. The xylosidase activity

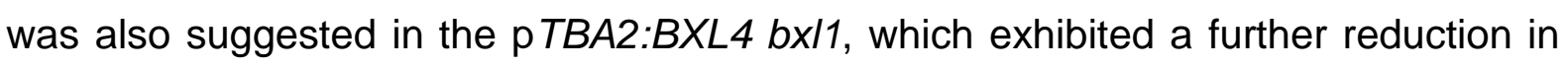
xylose levels compared to the wild type or control line (pTBA2:BXL1 bx/1). The greater reduction in xylose in pTBA2:BXL4 complemented bx/1 compared to pTBA2:BXL1 complemented line suggests the stronger xylosidase activity of BXL4 when expressed in mucilage. Recent findings by Ralet et al., (2016) suggest the presence of xylan side chains on RG-I that mediate the interaction with cellulose through non-covalent linkages. It is therefore possible that BXL4 acts on these xylan side chains. Mucilage is also thought to contain small quantities of the hemicelluloses xyloglucan and xylans with a $\beta$-1,4-linked D-xylose backbone (Ebringerová and Heinze, 2000; Voiniciuc et al., 2015; Hu et al., 2016). BXL4 could also act on these xylans whilst xyloglucan would not make a suitable substrate because of the $\alpha$-D-Xyl- $(1 \rightarrow 6)-\beta$-D-Glc (Zabotina, 2012) linkage which betaxylosidases cannot cleave. It is a well-known phenomenon that enzymes acting on polysaccharides show dual functions (Watt et al., 1998), and $B X L 4$ exhibits this dual function acting on chains of the two diastereomers $L$-arabinose and D-xylose. 
BXL4 acts upstream and downstream of JA signalling and contributes to camalexin production.

According to publicly available databases, $B X L 4$ is induced by infection with various plant pathogens. Our research findings concur with this, as BXL4 was upregulated upon infection with $B$. cinerea. It is known that $B$. cinerea infection in Arabidopsis results in transcriptional reprogramming of many genes (Sham et al., 2014). We further checked if $B X L 4$ was a tissue damage associated protein by mechanically wounding leaves of Arabidopsis. Mechanical wounding resulted in upregulation of $B X L 4$, in a JA-lle-dependent manner as the dde2-2 mutant, which is deficient in JA-lle (von Malek et al., 2002), did not reveal any significant $B X L 4$ upregulation upon wounding. Wounding is known to activate JA-lle production which in turn triggers expression of downstream genes involved in mitigatory responses (Turner et al., 2002). Pectin methylesterases are induced upon wounding in a JA-lle-dependant manner resulting in the formation of oligogalacturonides in dimer form (crosslinked with $\mathrm{Ca}^{2+}$ ) which elicit strong biological responses (Kohorn and Kohorn, 2012; Reymond et al., 2000; Bethke et al., 2014). It is plausible that $B X L 4$ is triggered by JA-lle after wounding to possibly render cell walls more recalcitrant to further damage as evidenced by the reduced induction of $B X L 4$ in the JA-lle-deficient mutant.

Our data shows that $b \times 14$ mutant Arabidopsis lines challenged with $B$. cinerea had a reduced expression of the JA-lle marker gene PDF1.2 together with decreased tissue abundance of JA and its biologically active derivative JA-lle. Our data suggest that bx/4 knockout mutants are compromised in JA-lle signalling upon infection. Oligogalacturonides regulate the expression of many genes involved in the JA-Ile biosynthesis pathway such as LOX3 and LOX4 (Denoux et al., 2008). The nature and quantity of the oligogalacturonides derived from $b x / 4$ mutants could impair their ability to activate the expression of the JA-lle biosynthetic genes. Predicted functional partners of BXL4 using STRING (version 11.0) (Szklarczyk et al., 2015) show its interaction with various pectate lyases and a polygalacturonase, which could be involved in the generation of elicitor active oligosaccharides. It is known that the modifications on oligosaccharides such as methylation and acetylation can influence the biological activity of oligosaccharides (Randoux et al., 2010) and that abundant arabinans can hinder polygalacturonase activity (Yoshioka et al., 1995). The bx/4 mutants could be producing fewer elicitor active oligogalacturonides. It is also known 
that many mutations involving cell wall related genes result in the alteration of phytohormone production or reprogramming of their signalling pathways (Ellis, 2002; Vega-Sanchez et al., 2012; Wang et al., 2017) which could also be the case with bx/4 mutants. Our $B$. cinerea infection data together with the wounding data suggests that $B X L 4$ is JA-lle dependent, and probably induces a positive feedback loop resulting in increased production of JA and JA-lle. It is known that JA-lle triggers the expression of its biosynthetic genes causing a positive feedback loop that promotes further JA-lle accumulation and subsequent activation of defence responses (Browse, 2009).

We also observe a reduced production of camalexin together with decreased expression of $P A D 3$ in the $b x / 4$ mutants after $B$. cinerea infection. This indicates that the oligosaccharides produced by the fungus when it breaches the cell wall or the plant itself might have a lower biological activity. Oligogalacturonides trigger downstream signalling pathways upon perception by wall associated kinases resulting in PAD3 induction and phytoalexin accumulation (Davis et al., 1986; Ferrari et al., 2007). It has also been revealed that camalexin biosynthesis is under the control of JA-lle signalling. Rowe et al., (2010) demonstrated that Arabidopsis mutants impaired in JA biosynthesis or perception show a reduced accumulation of camalexin after $B$. cinerea infection compared to wild type plants. Therefore, the reduced JA-lle production that is characteristic of our mutant after $B$. cinerea infection could also result in reduced camalexin accumulation.

\section{$B X L 4$ provides resistance to $B$. cinerea}

The $b x / 4$ mutants exhibited a compromised resistance to $B$. cinerea infection. Various mechanisms could be responsible for the compromised immunity such as the observed reduction in JA and JA-lle biosynthesis after infection. The production of JA upon cell wall degradation is a known defence response to necrotrophic pathogen attack (Turner et al., 2002; Laluk and Mengiste, 2010). JA-lle alters the pectin structure in response to wounding or attack from necrotrophic pathogens. Taurino et al., (2014) showed that JA-lle reduced the methylesterification of pectin in potato plants, which contributed to resistance to the necrotrophic pathogen Dickeya dadantii. Reduced methylesterification results in crosslinking of homogalacturonan with $\mathrm{Ca}^{2+}$. JA-lle also regulates the expression of $B X L 4$ as indicated in our wounding assays. Our $b x / 4$ mutants show an increased abundance of arabinans. Arabinans play a role in cell wall 
reinforcement, and in Arabidopsis, the interspacing of homogalacturonan with arabinan rich RG-I reduces crosslinking with $\mathrm{Ca}^{2+}$, making cell walls more flexible (Jones et al., 2003; Moore et al., 2008; Merced and Renzaglia, 2019). Reduction in arabinan levels in wild type plants could result in greater pectin crosslinking as compared to the arabinan rich bx/4 mutants, thus making the walls more recalcitrant reducing the penetration of fungal hyphae.

The bx/4 mutants also reveal a reduction in expression of $P A D 3$ and accumulation of the antimicrobial phytoalexin camalexin. Camalexin is known to play an important role in resistance to $B$. cinerea (Ferrari et al., 2007). Camalexin triggers fungal programmed cell death in $B$. cinerea minimising lesion development during early $B$. cinerea infection (Shlezinger et al., 2011). The reduction in the accumulation of camalexin in $b x / 4$ mutants contributes to enhanced susceptibility to the pathogen.

\section{CONCLUSION}

The results of this study reveal that $B X L 4$ is a putative cell wall localised bifunctional xylosidase/arabinofuranosidase that modifies cell wall polysaccharides and contributes to immunity against $B$. cinerea.

\section{ACKNOWLEDGEMENTS}

This work was supported by German Research Foundation (DFG, IRTG 2172 PRoTECT to T.I.) and the Studienstiftung des deutschen Volkes (stipend to P.S.). We would like to thank Prof. Dr. Volker Lipka for useful discussions and Franziska Kretzschmar for proofreading the manuscript.

\section{AUTHOR CONTRIBUTIONS}

A.G., G.H., and T.I. designed the research, A.G., R.M., D.H., P.S., and K.Z. performed the experiments. M.W., G.H., and T.I. provided the tools and materials. A.G., and T.I wrote the manuscript with contributions from the other authors.

\section{REFERENCES}

Arsovski, A.A., Haughn, G.W., and Western, T.L. (2010). Seed coat mucilage cells of Arabidopsis thaliana as a model for plant cell wall research. Plant Signal. 
Behav. 5: 796-801.

Arsovski, A.A., Popma, T.M., Haughn, G.W., Carpita, N.C., McCann, M.C., and Western, T.L. (2009). AtBXL1 encodes a bifunctional beta-D-xylosidase/alpha-Larabinofuranosidase required for pectic arabinan modification in Arabidopsis mucilage secretory cells. Plant Physiol. 150: 1219-34.

Benedetti, M., Verrascina, I., Pontiggia, D., Locci, F., Mattei, B., De Lorenzo, G., and Cervone, F. (2018). Four Arabidopsis berberine bridge enzyme-like proteins are specific oxidases that inactivate the elicitor-active oligogalacturonides. Plant J. 94: 260-273.

Bethke, G., Grundman, R.E., Sreekanta, S., Truman, W., Katagiri, F., and Glazebrook, J. (2014). Arabidopsis PECTIN METHYLESTERASEs contribute to immunity against Pseudomonas syringae. Plant Physiol. 164: 1093-107.

Bethke, G., Thao, A., Xiong, G., Li, B., Soltis, N.E., Hatsugai, N., Hillmer, R.A., Katagiri, F., Kliebenstein, D.J., Pauly, M., and Glazebrook, J. (2016). Pectin Biosynthesis Is Critical for Cell Wall Integrity and Immunity in Arabidopsis thaliana. Plant Cell 28: 537-56.

Breitenbach, H.H., Wenig, M., Wittek, F., Jordá, L., Maldonado-Alconada, A.M., Sarioglu, H., Colby, T., Knappe, C., BichImeier, M., Pabst, E. and Mackey, D. (2014). Contrasting Roles of the Apoplastic Aspartyl Protease APOPLASTIC, ENHANCED DISEASE SUSCEPTIBILITY1-DEPENDENT1 and LEGUME LECTIN-LIKE PROTEIN1 in Arabidopsis Systemic Acquired Resistance,. Plant Physiol. 165: 791-809.

Browse, J. (2009). Jasmonate Passes Muster: A Receptor and Targets for the Defense Hormone. Annu. Rev. Plant Biol. 60: 183-205.

Carpita, N.C. (2011). Update on mechanisms of plant cell wall biosynthesis: how plants make cellulose and other (1->4)- $\beta$-D-glycans. Plant Physiol. 155: 171-84. Chung, H.S., Koo, A.J.K., Gao, X., Jayanty, S., Thines, B., Jones, A.D., and Howe, G.A. (2008). Regulation and Function of Arabidopsis JASMONATE ZIM-Domain Genes in Response to Wounding and Herbivory. Plant Physiol. 146: 952-964.

Clough, S.J. and Bent, A.F. (1998). Floral dip: a simplified method for Agrobacteriummediated transformation of Arabidopsis thaliana. Plant J. 16: 735-43.

Davis, K.R., Darvill, A.G., Albersheim, P., and Dell, A. (1986). Host-Pathogen Interactions: XXIX. Oligogalacturonides Released from Sodium Polypectate by 
Endopolygalacturonic Acid Lyase Are Elicitors of Phytoalexins in Soybean. Plant Physiol. 80: 568-577.

Dean, G.H., Zheng, H., Tewari, J., Huang, J., Young, D.S., Hwang, Y.T., Western, T.L., Carpita, N.C., McCann, M.C., Mansfield, S.D., and Haughn, G.W. (2007). The Arabidopsis MUM2 gene encodes a beta-galactosidase required for the production of seed coat mucilage with correct hydration properties. Plant Cell 19: 4007-21.

Denoux, C., Galletti, R., Mammarella, N., Gopalan, S., Werck, D., De Lorenzo, G., Ferrari, S., Ausubel, F.M., and Dewdney, J. (2008). Activation of Defense Response Pathways by OGs and Flg22 Elicitors in Arabidopsis Seedlings. Mol. Plant 1: 423-445.

Ebringerová, A. and Heinze, T. (2000). Xylan and xylan derivatives - biopolymers with valuable properties, 1. Naturally occurring xylans structures, isolation procedures and properties. Macromol. Rapid Commun. 21: 542-556.

Ellis, C. (2002). The Arabidopsis Mutant cev1 Links Cell Wall Signaling to Jasmonate and Ethylene Responses. Plant Cell 14: 1557-1566.

Engelsdorf, T., Will, C., Hofmann, J., Schmitt, C., Merritt, B.B., Rieger, L., Frenger, M.S., Marschall, A., Franke, R.B., Pattathil, S., and Voll, L.M. (2017). Cell wall composition and penetration resistance against the fungal pathogen Colletotrichum higginsianum are affected by impaired starch turnover in Arabidopsis mutants. J. Exp. Bot. 68: 701-713.

Ettenauer, J., Piñar, G., Tafer, H., and Sterflinger, K. (2014). Quantification of fungal abundance on cultural heritage using real time PCR targeting the $\beta$-actin gene. Front. Microbiol. 5: 262.

Feldmann, K.A. (1991). T-DNA insertion mutagenesis in Arabidopsis: mutational spectrum. Plant J. 1: 71-82.

Ferrari, S. (2003). Tandemly Duplicated Arabidopsis Genes That Encode Polygalacturonase-Inhibiting Proteins Are Regulated Coordinately by Different Signal Transduction Pathways in Response to Fungal Infection. Plant Cell 15: 93106.

Ferrari, S., Galletti, R., Denoux, C., De Lorenzo, G., Ausubel, F.M., and Dewdney, J. (2007). Resistance to Botrytis cinerea induced in Arabidopsis by elicitors is independent of salicylic acid, ethylene, or jasmonate signaling but requires 
PHYTOALEXIN DEFICIENT3. Plant Physiol. 144: 367-79.

Ferrari, S., Plotnikova, J.M., De Lorenzo, G., and Ausubel, F.M. (2003). Arabidopsis local resistance to Botrytis cinerea involves salicylic acid and camalexin and requires EDS4 and PAD2, but not SID2, EDS5 or PAD4. Plant J. 35: 193-205.

Fry, S.C. (2004). Primary cell wall metabolism: tracking the careers of wall polymers in living plant cells. New Phytol. 161: 641-675.

Galletti, R., Ferrari, S., and De Lorenzo, G. (2011). Arabidopsis MPK3 and MPK6 play different roles in basal and oligogalacturonide- or flagellin-induced resistance against Botrytis cinerea. Plant Physiol. 157: 804-14.

Gille, S., Hänsel, U., Ziemann, M., and Pauly, M. (2009). Identification of plant cell wall mutants by means of a forward chemical genetic approach using hydrolases. Proc. Natl. Acad. Sci. U. S. A. 106: 14699-704.

Glass, N.L., Schmoll, M., Cate, J.H.D., and Coradetti, S. (2013). Plant cell wall deconstruction by ascomycete fungi. Annu. Rev. Microbiol. 67: 477-98.

Goujon, T., Minic, Z., El Amrani, A., Lerouxel, O., Aletti, E., Lapierre, C., Joseleau, J.-P., and Jouanin, L. (2003). AtBXL1, a novel higher plant (Arabidopsis thaliana) putative beta-xylosidase gene, is involved in secondary cell wall metabolism and plant development. Plant J. 33: 677-90.

Harholt, J., Suttangkakul, A., and Vibe Scheller, H. (2010). Biosynthesis of Pectin. Plant Physiol. 153: 384-395.

Haughn, G.W. and Western, T.L. (2012). Arabidopsis Seed Coat Mucilage is a Specialized Cell Wall that Can be Used as a Model for Genetic Analysis of Plant Cell Wall Structure and Function. Front. Plant Sci. 3: 64.

Hu, R., Li, J., Wang, X., Zhao, X., Yang, X., Tang, Q., He, G., Zhou, G., and Kong, Y. (2016). Xylan synthesized by Irregular Xylem 14 ( IRX14) maintains the structure of seed coat mucilage in Arabidopsis. J. Exp. Bot. 67: 1243-1257.

Iven, T., König, S., Singh, S., Braus-Stromeyer, S.A., Bischoff, M., Tietze, L.F., Braus, G.H., Lipka, V., Feussner, I., and Dröge-Laser, W. (2012). Transcriptional activation and production of tryptophan-derived secondary metabolites in arabidopsis roots contributes to the defense against the fungal vascular pathogen Verticillium longisporum. Mol. Plant 5: 1389-402.

Jones, L., Milne, J.L., Ashford, D., and McQueen-Mason, S.J. (2003). Cell wall 
arabinan is essential for guard cell function. Proc. Natl. Acad. Sci. 100: 1178311788.

Kohorn, B.D. and Kohorn, S.L. (2012). The cell wall-associated kinases, WAKs, as pectin receptors. Front. Plant Sci. 3: 88.

Kusch, S., Thiery, S., Reinstädler, A., Gruner, K., Zienkiewicz, K., Feussner, I., and Panstruga, R. (2019). Arabidopsis mlo3 mutant plants exhibit spontaneous callose deposition and signs of early leaf senescence. Plant Mol. Biol.

Laluk, K. and Mengiste, T. (2010). Necrotroph attacks on plants: wanton destruction or covert extortion? Arab. B. 8: e0136.

Li, X., Lassner, M., and Zhang, Y. (2002). Deleteagene: A Fast Neutron Deletion Mutagenesis-Based Gene Knockout System for Plants. Comp. Funct. Genomics 3: $158-160$.

Liepman, A.H., Wightman, R., Geshi, N., Turner, S.R., and Scheller, H.V. (2010). Arabidopsis - a powerful model system for plant cell wall research. Plant J. 61: 1107-21.

Lionetti, V., Raiola, A., Camardella, L., Giovane, A., Obel, N., Pauly, M., Favaron, F., Cervone, F., and Bellincampi, D. (2007). Overexpression of Pectin Methylesterase Inhibitors in Arabidopsis Restricts Fungal Infection by Botrytis cinerea. Plant Physiol. 143: 1871-1880.

Liu, N., Sun, Y., Pei, Y., Zhang, X., Wang, P., Li, X., Li, F., and Hou, Y. (2018). A Pectin Methylesterase Inhibitor Enhances Resistance to Verticillium Wilt. Plant Physiol. 176: 2202-2220.

von Malek, B., van der Graaff, E., Schneitz, K., and Keller, B. (2002). The Arabidopsis male-sterile mutant dde2-2 is defective in the ALLENE OXIDE SYNTHASE gene encoding one of the key enzymes of the jasmonic acid biosynthesis pathway. Planta 216: 187-92.

Manabe, Y., Nafisi, M., Verhertbruggen, Y., Orfila, C., Gille, S., Rautengarten, C., Cherk, C., Marcus, S.E., Somerville, S., Pauly, M. and Knox, J.P. (2011). Lossof-function mutation of REDUCED WALL ACETYLATION2 in Arabidopsis leads to reduced cell wall acetylation and increased resistance to Botrytis cinerea. Plant Physiol. 155: 1068-78.

Merced, A. and Renzaglia, K.S. (2019). Contrasting pectin polymers in guard cell walls of Arabidopsis and the hornwort Phaeoceros reflect physiological 
differences. Ann. Bot. 123: 579-585.

Minic, Z. (2004). Purification and Characterization of Enzymes Exhibiting -DXylosidase Activities in Stem Tissues of Arabidopsis. Plant Physiol. 135: 867878.

Mohnen, D., Bar-Peled, M. and Somerville, C. (2008). Biosynthesis of Plant Cell Walls. In Biomass Recalcitrance, M. Himmel, ed (Blackwell Publishing, Oxford), pp. 94-187.

Mohnen, D. (2008). Pectin structure and biosynthesis. Curr. Opin. Plant Biol. 11: 26677.

Moore, J.P., Farrant, J.M., and Driouich, A. (2008). A role for pectin-associated arabinans in maintaining the flexibility of the plant cell wall during water deficit stress. Plant Signal. Behav. 3: 102-4.

Nakagawa, T., Nakamura, S., Tanaka, K., Kawamukai, M., Suzuki, T., Nakamura, K., Kimura, T., and Ishiguro, S. (2008). Development of R4 gateway binary vectors (R4pGWB) enabling high-throughput promoter swapping for plant research. Biosci. Biotechnol. Biochem. 72: 624-9.

Nie, P., Li, X., Wang, S., Guo, J., Zhao, H., and Niu, D. (2017). Induced Systemic Resistance against Botrytis cinerea by Bacillus cereus AR156 through a JA/ETand NPR1-Dependent Signaling Pathway and Activates PAMP-Triggered Immunity in Arabidopsis. Front. Plant Sci. 8.

Noguchi, K., Yasumori, M., Imai, T., Naito, S., Matsunaga, T., Oda, H., Hayashi, H., Chino, M., and Fujiwara, T. (1997). bor1-1, an Arabidopsis thaliana Mutant That Requires a High Level of Boron. Plant Physiol. 115: 901-906.

O'Neill, M., Albersheim, P., and Darvill, A. (1990). The Pectic Polysaccharides of Primary Cell Walls. In Methods in plant biochemistry, pp. 415-441.

Passardi, F., Penel, C., and Dunand, C. (2004). Performing the paradoxical: how plant peroxidases modify the cell wall. Trends Plant Sci. 9: 534-540.

Penninckx, I.A., Thomma, B.P., Buchala, A., Métraux, J.P. and Broekaert, W.F. (1998). Concomitant Activation of Jasmonate and Ethylene Response Pathways Is Required for Induction of a Plant Defensin Gene in Arabidopsis. Plant Cell 10: 2103-2114.

Quoc, N.B. and Bao Chau, N.N. (2017). The Role of Cell Wall Degrading Enzymes in Pathogenesis of Magnaporthe oryzae. Curr. Protein Pept. Sci. 18. 
Ralet, M.-C., Crépeau, M.-J., Vigouroux, J., Tran, J., Berger, A., Sallé, C., Granier, F., Botran, L., and North, H.M. (2016). Xylans Provide the Structural Driving Force for Mucilage Adhesion to the Arabidopsis Seed Coat. Plant Physiol. 171: 165-178.

Randoux, B., Renard-Merlier, D., Mulard, G., Rossard, S., Duyme, F., Sanssené, J., Courtois, J., Durand, R., and Reignault, P. (2010). Distinct defenses induced in wheat against powdery mildew by acetylated and nonacetylated oligogalacturonides. Phytopathology 100: 1352-63.

Reymond, P., Weber, H., Damond, M., and Farmer, E.E. (2000). Differential gene expression in response to mechanical wounding and insect feeding in Arabidopsis. Plant Cell 12: 707-20.

Ridley, B.L., O'Neill, M.A., and Mohnen, D. (2001). Pectins: structure, biosynthesis, and oligogalacturonide-related signaling. Phytochemistry 57: 929-67.

Rowe, H.C., Walley, J.W., Corwin, J., Chan, E.K.-F., Dehesh, K., and Kliebenstein, D.J. (2010). Deficiencies in Jasmonate-Mediated Plant Defense Reveal Quantitative Variation in Botrytis cinerea Pathogenesis. PLoS Pathog. 6: e1000861.

Scalschi, L., Sanmartín, M., Camañes, G., Troncho, P., Sánchez-Serrano, J.J., García-Agustín, P., and Vicedo, B. (2015). Silencing of OPR3 in tomato reveals the role of OPDA in callose deposition during the activation of defense responses against Botrytis cinerea. Plant J. 81: 304-315.

Scheller, H.V. and Ulvskov, P. (2010). Hemicelluloses. Annu. Rev. Plant Biol. 61: 263-89.

Schuhegger, R., Nafisi, M., Mansourova, M., Petersen, B.L., Olsen, C.E., Svatos, A., Halkier, B.A., and Glawischnig, E. (2006). CYP71B15 (PAD3) Catalyzes the Final Step in Camalexin Biosynthesis. Plant Physiol. 141: 1248-1254.

Sham, A., Al-Azzawi, A., Al-Ameri, S., Al-Mahmoud, B., Awwad, F., AlRawashdeh, A., Iratni, R., and AbuQamar, S. (2014). Transcriptome analysis reveals genes commonly induced by Botrytis cinerea infection, cold, drought and oxidative stresses in Arabidopsis. PLoS One 9: e113718.

Shlezinger, N., Minz, A., Gur, Y., Hatam, I., Dagdas, Y.F., Talbot, N.J., and Sharon, A. (2011). Anti-Apoptotic Machinery Protects the Necrotrophic Fungus Botrytis cinerea from Host-Induced Apoptotic-Like Cell Death during Plant Infection. PLoS 
Pathog. 7: e1002185.

Somerville, C. (2006). Cellulose synthesis in higher plants. Annu. Rev. Cell Dev. Biol. 22: 53-78.

Sommer-Knudsen, J., Bacic, A., and Clarke, A.E. (1998). Hydroxyproline-rich plant glycoproteins. Phytochemistry 47: 483-497.

Staats, M. and van Kan, J.A.L. (2012). Genome Update of Botrytis cinerea Strains B05.10 and T4. Eukaryot. Cell 11: 1413-1414.

Szklarczyk, D., Franceschini, A., Wyder, S., Forslund, K., Heller, D., HuertaCepas, J., Simonovic, M., Roth, A., Santos, A., Tsafou, K.P. and Kuhn, M. (2015). STRING v10: protein-protein interaction networks, integrated over the tree of life. Nucleic Acids Res. 43: D447-D452.

Taurino, M., Abelenda, J.A., Río-Alvarez, I., Navarro, C., Vicedo, B., Farmaki, T., Jiménez, P., García-Agustín, P., López-Solanilla, E., Prat, S. and Rojo, E. (2014). Jasmonate-dependent modifications of the pectin matrix during potato development function as a defense mechanism targeted by Dickeya dadantii virulence factors. Plant J. 77: 418-429.

Thines, B., Katsir, L., Melotto, M., Niu, Y., Mandaokar, A., Liu, G., Nomura, K., He, S.Y., Howe, G.A., and Browse, J. (2007). JAZ repressor proteins are targets of the SCF(COI1) complex during jasmonate signalling. Nature 448: 661-5.

Tsai, A.Y.-L., Kunieda, T., Rogalski, J., Foster, L.J., Ellis, B.E., and Haughn, G.W. (2017). Identification and Characterization of Arabidopsis Seed Coat Mucilage Proteins. Plant Physiol. 173: 1059-1074.

Turner, J.G., Ellis, C., and Devoto, A. (2002). The Jasmonate Signal Pathway. Plant Cell 14: S153-S164.

Underwood, W. (2012). The plant cell wall: a dynamic barrier against pathogen invasion.Underwood, W. (2012) "The plant cell wall: a dynamic barrier against pathogen invasion.”, Frontiers in plant science, Front. Plant Sci. 3: 85.

Vega-Sanchez, M.E., Verhertbruggen, Y., Christensen, U., Chen, X., Sharma, V., Varanasi, P., Jobling, S.A., Talbot, M., White, R.G., Joo, M. and Singh, S. (2012). Loss of Cellulose Synthase-Like F6 Function Affects Mixed-Linkage Glucan Deposition, Cell Wall Mechanical Properties, and Defense Responses in Vegetative Tissues of Rice. Plant Physiol. 159: 56-69.

Vogel, H. (1956). A Convenient Growth Medium for Neurospora crassa. Microb. 
Genet. Bullletin: 42-47.

Vogel, J.P. (2002). PMR6, a Pectate Lyase-Like Gene Required for Powdery Mildew Susceptibility in Arabidopsis. Plant Cell 14: 2095-2106.

Vogel, J.P., Raab, T.K., Somerville, C.R., and Somerville, S.C. (2004). Mutations in PMR5 result in powdery mildew resistance and altered cell wall composition. Plant J. 40: 968-78.

Voiniciuc, C., Guenl, M., Schmidt, M.H.-W., and Usadel, B. (2015). Highly Branched Xylan Made by IRX14 and MUCl21 Links Mucilage to Arabidopsis Seeds. Plant Physiol.: pp.01441.2015.

Vorwerk, S., Somerville, S., and Somerville, C. (2004). The role of plant cell wall polysaccharide composition in disease resistance. Trends Plant Sci. 9: 203-209.

Wang, X., Hou, S., Wu, Q., Lin, M., Acharya, B.R., Wu, D., and Zhang, W. (2017). IDL6-HAE/HSL2 impacts pectin degradation and resistance to Pseudomonas syringae pv tomato DC3000 in Arabidopsis leaves. Plant J. 89: 250-263.

Watt, D.K., Ono, H., and Hayashi, K. (1998). Agrobacterium tumefaciens betaglucosidase is also an effective beta-xylosidase, and has a high transglycosylation activity in the presence of alcohols. Biochim. Biophys. Acta 1385: 78-88.

Western, T.L., Skinner, D.J., and Haughn, G.W. (2000). Differentiation of Mucilage Secretory Cells of the Arabidopsis Seed Coat. Plant Physiol. 122: 345-356.

Willats, W.G., Orfila, C., Limberg, G., Buchholt, H.C., van Alebeek, G.J., Voragen, A.G., Marcus, S.E., Christensen, T.M., Mikkelsen, J.D., Murray, B.S., and Knox, J.P. (2001). Modulation of the degree and pattern of methyl-esterification of pectic homogalacturonan in plant cell walls. Implications for pectin methyl esterase action, matrix properties, and cell adhesion. J. Biol. Chem. 276: 1940413.

Yan, J., Zhang, C., Gu, M., Bai, Z., Zhang, W., Qi, T., Cheng, Z., Peng, W., Luo, H., Nan, F., Wang, Z., and Xie, D. (2009). The Arabidopsis CORONATINE INSENSITIVE1 protein is a jasmonate receptor. Plant Cell 21: 2220-36.

Yan, Y., Stolz, S., Chetelat, A., Reymond, P., Pagni, M., Dubugnon, L., and Farmer, E.E. (2007). A Downstream Mediator in the Growth Repression Limb of the Jasmonate Pathway. Plant Cell 19: 2470-2483.

Yeoh, S., Shi, J., and Langrish, T.A.G. (2008). Comparisons between different 
techniques for water-based extraction of pectin from orange peels. Desalination 218: 229-237.

Yoshioka, H., Kashimura, Y., and Kaneko, K. (1995). beta;-D-Galactosidase and alpha;-L-Arabinofuranosidase Activities during the Softening of Apples. J. Japanese Soc. Hortic. Sci. 63: 871-878.

Zabotina, O.A. (2012). Xyloglucan and Its Biosynthesis. Front. Plant Sci. 3.

Zarei, A., Körbes, A.P., Younessi, P., Montiel, G., Champion, A., and Memelink, J. (2011). Two GCC boxes and AP2/ERF-domain transcription factor ORA59 in jasmonate/ethylene-mediated activation of the PDF1.2 promoter in Arabidopsis. Plant Mol. Biol. 75: 321-31.

Zhang, H., Hong, Y., Huang, L., Li, D., and Song, F. (2016). Arabidopsis AtERF014 acts as a dual regulator that differentially modulates immunity against Pseudomonas syringae pv. tomato and Botrytis cinerea. Sci. Rep. 6: 30251.

Zhou, N. (1999). Arabidopsis PAD3, a Gene Required for Camalexin Biosynthesis, Encodes a Putative Cytochrome P450 Monooxygenase. Plant Cell 11: 24192428. 

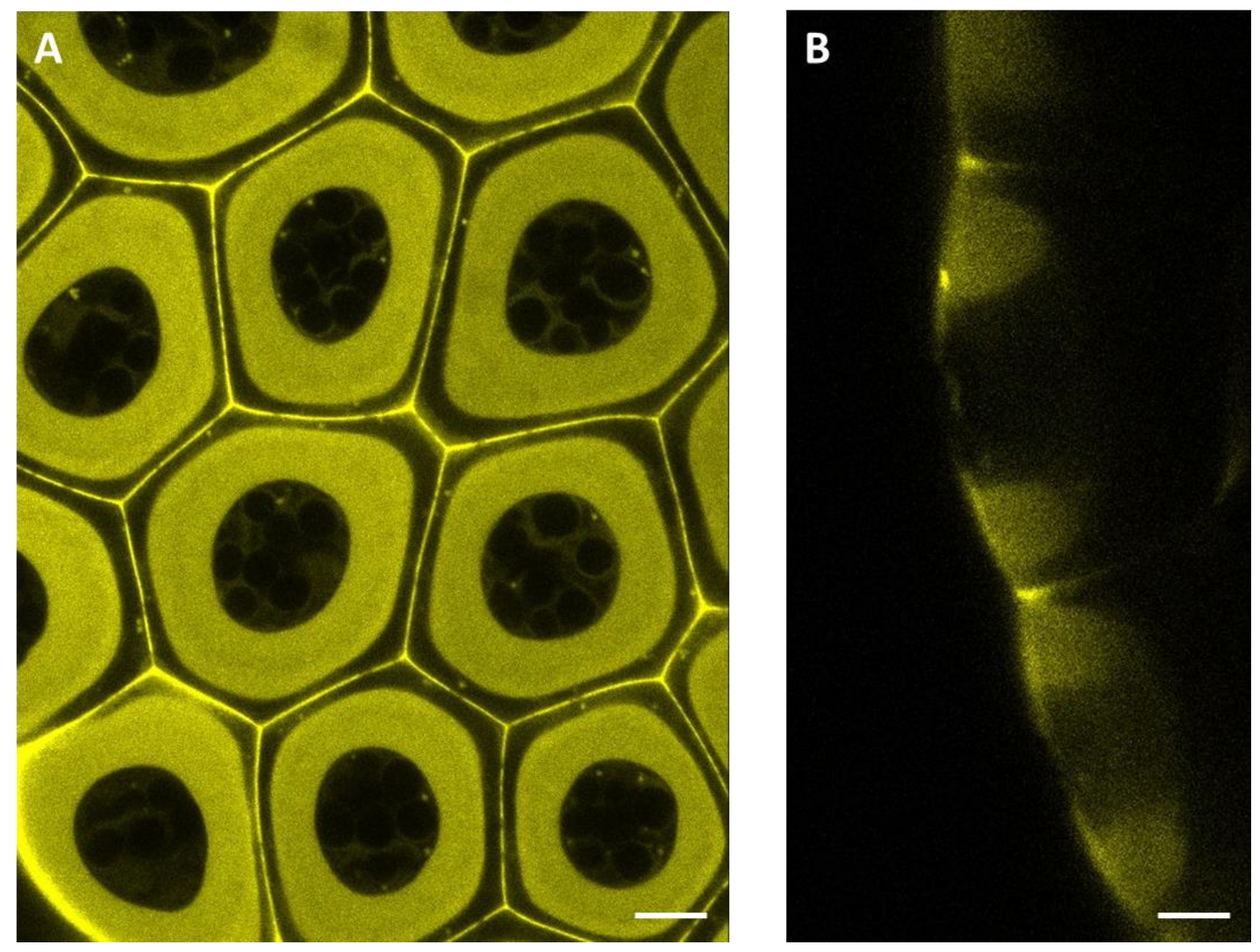

Supplementary Figure 1: BXL4-CITRINE localises to the apoplast in Arabidopsis seed coat epidermal cells

(A) BXL1-CITRINE stably expressed under the TBA2 promoter localises to the apoplast of Arabidopsis seed coat epidermal cells 7 days post anthesis (dpa). Single plane images of the seed coats were obtained by confocal microscopy. The apopolast of the of seed coat epidermal cells appears like a doughnut ring that surrounds the cytoplast when imaged from the top. (B) Visualisation of the seed coat epidermal cells from the side at 7 dpa shows the apoplast as two pockets in the apical corners of the cells (arrow). Scale bars, $10 \mu \mathrm{m}$ 

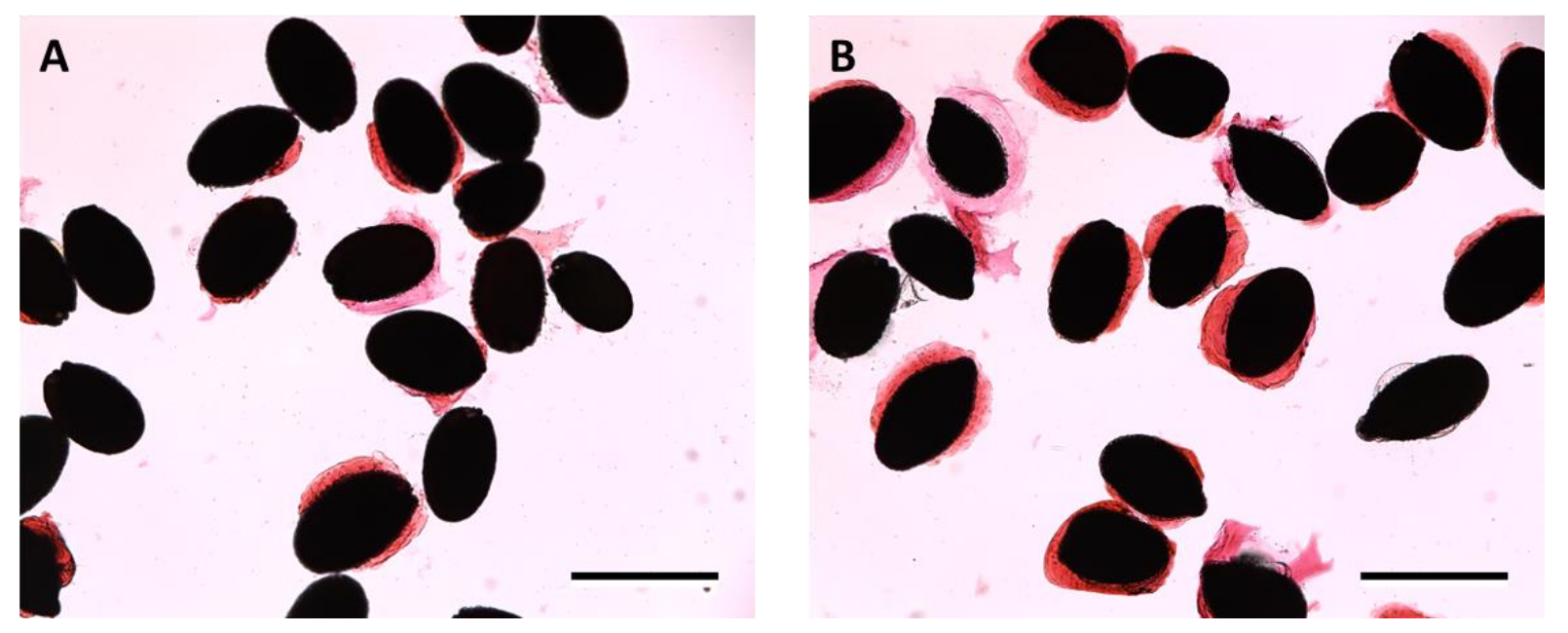

Supplementary Figure 2: pTBA2:BXL6 expressed in bxl1 does not rescue mucilage extrusion phenotype.

$\mathrm{p} T B A 2: B X L 6$ when expressed in $b x / 1$ fails to rescue mucilage extrusion as shown by complemented line $567(A)$ and line $572(B)$. Scale bar $300 \mu \mathrm{m}$ 
A

JA

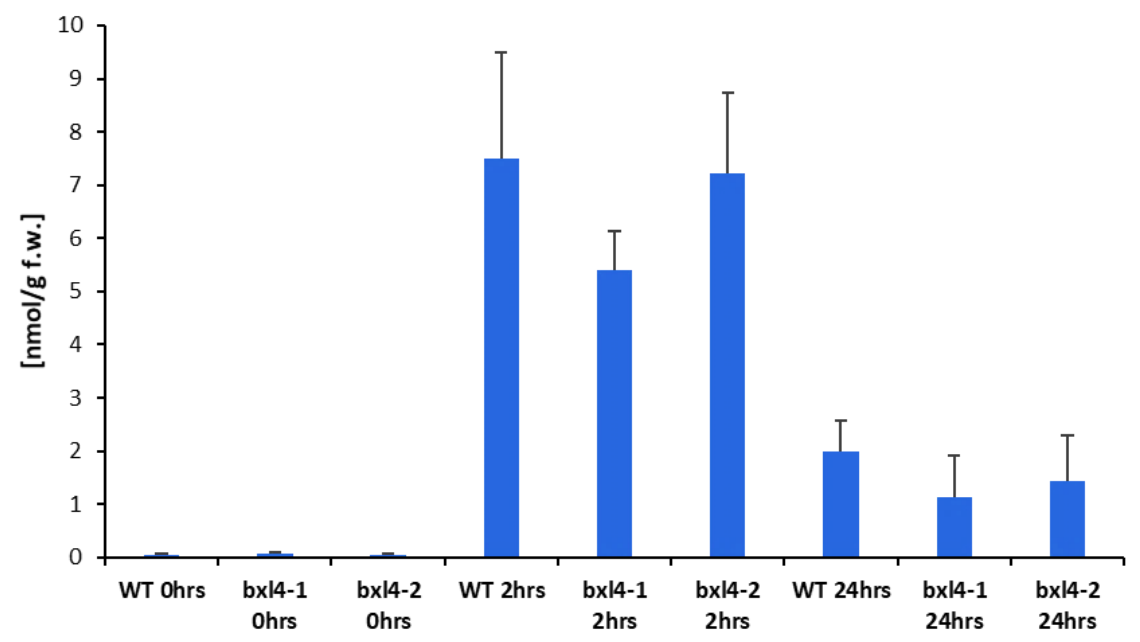

B

JA-Ile

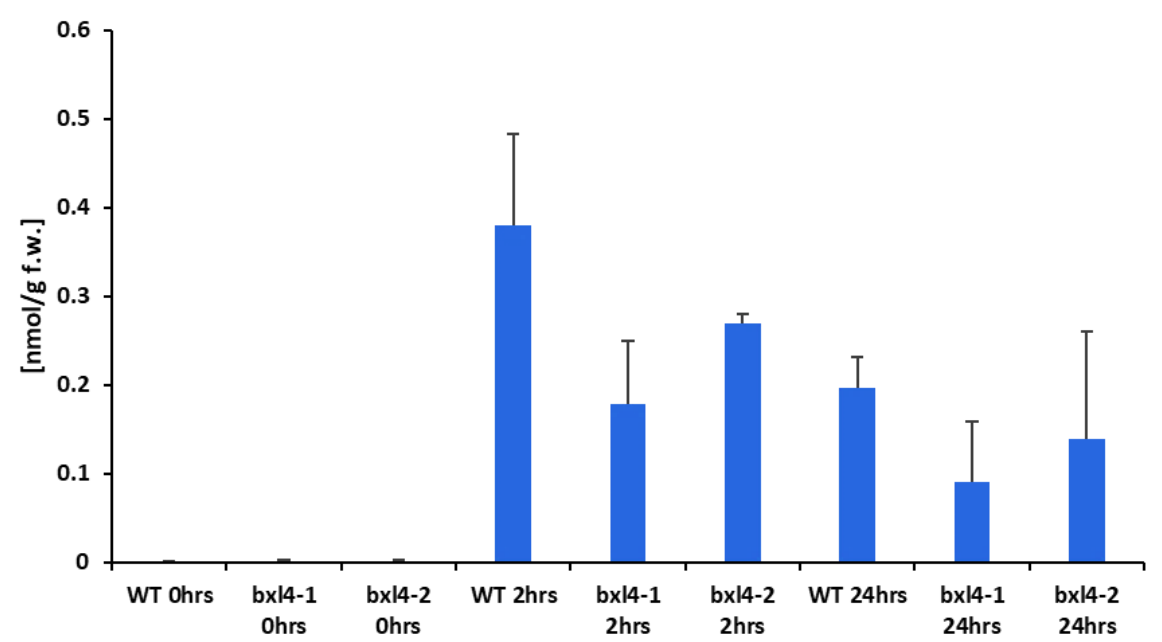

Supplementary Figure 3: JA and JA-lle accumulation after wounding in Arabidopsis

Col- 0 and $b x / 4$ mutant lines were mechanically wounded and leaves were sampled at 0,2 and $24 \mathrm{~h}$ after infection. Extracted levels of JA (A), JA-lle (B) were analysed using nanoelectrospray coupled to a tandem mass spectrometer. Error bars represent standard deviation of 3 biological replicates 
Supplementary Table 1: List of primers

\begin{tabular}{|c|c|c|}
\hline $\begin{array}{l}\text { Cloning } \\
\text { primers }\end{array}$ & Forward 5'-3' & Reverse 5'-3' \\
\hline BXL1 & $\begin{array}{l}\text { GGGGACAAGTTTGTACAAAAAAGCAGGC } \\
\text { TATGTCTTGTTATAATAAAGCACTATTG }\end{array}$ & $\begin{array}{l}\text { GGGGACCACTTTGTACAAGAAAGCTGGGT } \\
\text { AAAGTTGCGGTTGGACCAA }\end{array}$ \\
\hline BXL4 & $\begin{array}{l}\text { GGGGACAAGTTTGTACAAAAAAGCAGGC } \\
\text { TTGGGCTCTTCTTCTCCATTA }\end{array}$ & $\begin{array}{l}\text { GGGGACCACTTTGTACAAGAAAGCTGGGT } \\
\text { AGATTCTAATGCTTAAGGAATGTTTTA }\end{array}$ \\
\hline BXL6 & $\begin{array}{l}\text { GGGGACAAGTTTGTACAAAAAAGCAGGC } \\
\text { T ATGAATCTTCAGTTGACTCTAATC }\end{array}$ & $\begin{array}{l}\text { GGGGACCACTTTGTACAAGAAAGCTGGGT } \\
\text { AGAATTCAACAGAGAGAGAATGT }\end{array}$ \\
\hline $\begin{array}{l}\text { Genotypin } \\
\text { g primers }\end{array}$ & LB & RB \\
\hline$b x / 1$ & ATTTTGCCGATTTCGGAAC & AACCGTCGCGTCGGCTTCAC \\
\hline$b x / 4-1$ & ATTTTGCCGATTTCGGAAC & ATCTCCGACATGAAGAAGATGC \\
\hline$b x \mid 4-2$ & $\begin{array}{l}\text { TAGCATCTGAATTTCATAACCAATCTCGAT } \\
\text { AC }\end{array}$ & ATCTCCGACATGAAGAAGATGC \\
\hline$b x / 6$ & ATTTTGCCGATTTCGGAAC & TACCACAGCATTGAAGTCGTATC \\
\hline $\begin{array}{l}\text { qRT-PCR } \\
\text { primers }\end{array}$ & Forward 5'-3' & Reverse 5'-3' \\
\hline BXL4 & ATACACAACACCACTACAAGGAC & ATCTCCGACATGAAGAAGATGC \\
\hline PDF1.2 & TTGCTGCTTTCGACGCA & TGTCCCACTTGGCTTCTCG \\
\hline JAZ10 & ATCCCGATTTCTCCGGTCCA & $\begin{array}{l}\text { ACTTTCTCCTTGCGATGGGAAGA (Benthke } \\
\text { et al., 2016) }\end{array}$ \\
\hline PAD3 & TGCTCTCAAGTTCACCACT & $\begin{array}{l}\text { CGAATCTCGTCTTGCACTT (Benthke et al., } \\
\text { 2016) }\end{array}$ \\
\hline ACTIN8 & GGTTTTCCCCAGTGTTGTTG & CTCCATGTCATCCCAGTTGC \\
\hline $\begin{array}{l}\text { Botrytis } \\
\text { ACTIN }\end{array}$ & TGGAGATGAAGCGCAATCCA & AAGCGTAAAGGGAGAGGACG \\
\hline $\begin{array}{l}\text { Botrytis } \\
\text { Tubulin }\end{array}$ & CCGTCATGTCCGGTGTTAC & CGACCGTTACGGAAATCGG \\
\hline
\end{tabular}


Supplementary Table 2: Mass transitions and corresponding conditions for determination of the phytohormones.

\begin{tabular}{|c|c|c|c|c|c|}
\hline \multicolumn{2}{|c|}{$\begin{array}{c}\text { MRM } \\
\text { Transitions }\end{array}$} & \multirow{2}{*}{ Analyte } & \multirow{2}{*}{$\begin{array}{c}\text { DP } \\
\text { [declustering } \\
\text { potential] }\end{array}$} & \multirow{2}{*}{$\begin{array}{c}\text { EP } \\
\text { [entrance } \\
\text { potential] }\end{array}$} & \multirow{2}{*}{$\begin{array}{c}\text { CE } \\
\text { [collisior } \\
\text { energy] }\end{array}$} \\
\hline Q1 & Q3 & & & & \\
\hline 209 & 59 & $\mathrm{JA}$ & -30 & -4.5 & -24 \\
\hline 225 & 59 & $11,12-\mathrm{OH}-\mathrm{JA}$ & -35 & -9 & -28 \\
\hline 263 & 165 & dinor-oPDA & -40 & -5 & -20 \\
\hline 296 & 170.2 & D5-oPDA & -65 & -4 & -28 \\
\hline 305 & 97 & $12-\mathrm{HSO}_{4}-\mathrm{JA}$ & -30 & -4 & -32 \\
\hline 308 & 116 & JA-Val & -45 & -5 & -28 \\
\hline 322 & 130 & JA-Ile/Leu & -45 & -5 & -28 \\
\hline 325 & 133 & D4-JA-Leu & -80 & -4 & -30 \\
\hline 324 & 116 & $12 \mathrm{OH}-\mathrm{JA}-\mathrm{Val}$ & -45 & -10 & -30 \\
\hline 338 & 130 & 12OH-JA-Ile & -45 & -10 & -30 \\
\hline 352 & 130 & 12COOH-JA-lle & -45 & -10 & -30 \\
\hline 387 & 59 & 12-O-Gluc-JA & -85 & -9 & -59 \\
\hline
\end{tabular}




\section{Discussion}

\subsection{Pectin monosaccharide analysis using GC-MS}

Plant cell wall composition varies during development and in response to environmental changes. Plant attack by pathogens induces the differential regulation of many genes involved in remodelling of cell wall pectin. The effect of many proteins acting on pectin are not well understood given the complexity in cell wall analytics and the close interlinkages existing amongst cell wall polysaccharides. One objective of this project was to design a fast and easy method to determine pectin composition. Our protein of interest, BXL4, putatively acts on the pectin rhamnogalacturonan I therefore, we developed a method that could give a credible monosaccharide composition of pectin from various tissues.

Cell wall pectin analysis has been based on various strategies including immunolabeling using antibodies specific to different pectins. The limitation however is that most of these antibodies are large probes and consequently the cell walls have to be pre-processed to reduce masking of cell wall components and allow sufficient binding of the probes (Voiniciuc et al., 2018). Pre-processing leads to loss of some cell wall components and loss of valuable information. Immunolabeling may also lead to misleading conclusions for instance pectin labelling with $2 \mathrm{~F} 4$ which recognises demethylesterified, Ca2+ cross-linked homogalacturonan (Liners et al., 1989), requires addition of calcium ions (Liners and Van Cutsem, 1992), at levels higher than physiological level resulting in overestimation of presence of calcium cross linked homogalacturonan (Hocq et al., 2017). Click chemistry, which involves joining small sugar units to form a substance (DeBolt and Estevez, 2012), has been used for pectin analytics but if fails to give a monosaccharide composition profile of various pectins. Dyes have also been developed that stain certain pectin domains such as ruthenium red (Hanke and Northcote, 1975) and propidium iodide (Rounds et al., 2011) when used at low concentrations. However, ruthenium red staining of mucilage for instance can be affected by other external factors such as presence of calcium ions (Voiniciuc et al., 2013), whilst propidium iodide is only useful in living cells (Rounds et al., 2011). Other methods that have been used in cell wall analytics involve GC-MS and HPLC based methods (Biswal et al., 2017; Bethke and Glazebrook, 2019). The GC-MS 
based method involves either derivatisation of the sugars to alditol acetates which makes it impossible to detect uronic acids, or methanolysis which is a very timeconsuming process. Chromotograms derived from methanolysis of sugars are very difficult to analyse because one monosaccharide can generate several signals due to production of several analytes (Biswal et al., 2017).

Our method employs water extraction of pectin from various sources including mucilage, and the alcohol insoluble residue (AIR) derived from various tissues. The putative target pectin of our protein of interest BXL4 is RG-I, which is very abundant in Arabidopsis seed mucilage (Western et al., 2000). Hydration of Arabidopsis seeds leads to the extrusion of this mucilage, which can be easily extracted for analysis. Likewise, the agitation in hot water of an alcohol insoluble residue obtained from Arabidopsis leaves leads to the extraction of water-soluble pectin, as has been described previously (Vriesmann et al., 2011). In our protocol, the extracted pectin is hydrolysed with trifluoroacetic acid (TFA) followed by evaporation of the TFA (Li et al., 2018), eliminating the need for a neutralisation step, which precipitates most of the galacturonic acid. The hydrolysed sugars are sequentially derivatised using MOX and MSTFA, thus giving a reduction in preparation time. The method also allows for quantification of galacturonic acids, which is not possible when sugars are derivatised to alditol acetates (Merkle and Poppe, 1994). Our method gives another advantage of being able to simultaneously detect and quantify neutral sugars and galacturonic acid. The advantage of using mucilage model with our method is that the predominantly RG-I composition of mucilage allows for detection of subtle changes that might occur in the RG-I sugars. Data obtained using our method show that we can accurately quantify the relative composition of mucilage from Arabidopsis mutant $b x / 1$, Ws and Col-0 to levels that have been published. The arabinose composition in the bx/1 mutant shows a two-fold increase compared to wild type Ws, as described in previous work (Arsovski et al., 2009). As expected, rhamnose and galacturonic acid are the most abundant sugars, given that RG-I backbone consists of alternating galacturonic acid and rhamnose residues (Harholt et al., 2010). Our method also gives a reliable quantification of galacturonic acid measured in citrus peel pectin sourced from SigmaAldrich, thus providing further evidence of robustness. Analysis of pectin extracted from leaf AIR of Col-0 showed an abundance of galacturonic acid of more than $70 \%$ which is consistent to what has already been published (Mohnen, 2008). Our method 
is able to detect all the expected sugar residues, hence making it a useful tool for comparative analytics of all the various pectin moieties.

Overall, we were able to develop a robust GC-MS based method, which is reliable and time-saving to generate monosaccharide profiles of pectin from various sources. These monosaccharide profiles can be used for comparative purposes and provide an insight to the link between pectin composition and function.

\subsection{BXL4 could act on arabinans in the apoplast and its disruption does not affect plant morphology}

Previous work (Breitenbach et al., 2014; Goujon et al., 2003) and generated prediction using SignalP-5.0 (Almagro Armenteros et al., 2019) indicate that BXL4 has a predicted signal peptide for localisation in the extracellular matrix. We constructed a pTBA2:BXL4-CITRINE construct for expression in Arabidopsis seed coat epidermal cells. We expressed $B X L 4$ using a seed coat specific promoter TBA2 (TESTA ABUNDANT2), which had been identified in previous screens to be seed coat specific and highly expressed in Arabidopsis seed coats (Tsai et al., 2017). The Citrine-tagged BXL4 localised in the mucilage pocket at seven days post anthesis (Figure 1A \&B, Manuscript B). As the secretory pathway is likely conserved, our results indicate that BXL4 is likely also secreted to the apoplast in other tissues such as leaves. These results are therefore consistent with the finding of BXL4 in the cell wall of Arabidopsis plants expressing a P. syringae effector protein AvrRpm1 (Breitenbach et al., 2014). As mucilage is easy to investigate, the generated plant lines also gave us the opportunity to analyse a potential influence of BXL4 on cell wall composition. Mucilage is a highly pectinaceous substance made by the seed coat epidermal cells consisting predominantly of RG-I, and small amounts of homogalacturonan, cellulose and hemicellulose (Arsovski et al., 2010; Haughn and Western, 2012). It is known that Arabidopsis seed coat epidermal cells secrete mucilage together with mucilage modifying proteins to the apoplast between four and eight days post anthesis (Arsovski et al., 2010; Dean et al., 2007; Haughn and Western, 2012; Western et al., 2000).

Various proteins acting on mucilage components have already been confirmed to localise to the mucilage pocket. The beta-galactosidase MUM2, and xylosidase/arabinofuranosidase BXL1 acting on mucilage are deposited 
simultaneously with mucilage into the apoplast were they are thought to act on the side chains of RG-I (Dean et al., 2007; Arsovski et al., 2009). Knocking out either of the two genes results in mucilage that does not extrude properly, as it is lacking the correct chemical balance. Work by Arsovski et al., (2009) showed that mucilage from bx/1 knockout mutants has a higher abundance of (1-5)-linked arabinans relative to the wild type, thus indicating the arabinofuranosidase activity of BXL1. BXL4 is expressed at a low level in many organs, but it has not been identified in mucilage. Analysis of the protein structure indicates that it possesses glycosyl hydrolase domains (InterPRO-EMBL-EBI). Earlier work by Goujon et al., (2003) indicated that $B X L 4$ clustered together with $B X L 1$ in a small gene family of Arabidopsis betaxylosidases (BXLs) with seven members. The betaxylosidases are similar to those characterised in fungal and bacterial pathogens and involved in cell wall degradation (de Vries et al., 2000). The presence of glycosyl hydrolase domains and the localisation of BXL4 all point to a putative role in hydrolysing some polysaccharide component of the cell wall. Expression of TBA2 driven BXL4 in the mucilage of the $b x / 1$ mutant was able to rescue the mucilage phenotype suggesting that BXL4 and BXL1 are functionally similar (Figure 2D, Manuscript B). TBA2 is a very strong promoter, as it drives the most abundant seed coat specific protein TBA2 (Tsai et al., 2017). The strong expression could have also amplified the activity of BXL4 hence the rescue of the phenotype. It is also interesting that another member of the betaxylosidase family, BXL6 did not exhibit localisation in the mucilage and consequently it did not rescue the mucilage defect of bx/1 (Supplementary Figure 2, Manuscript $B$ ), thus demonstrating the different functional roles within the gene family. The spacial and temporal expression of $B X L 1$ and $B X L 4$ is differentially regulated (Goujon et al., 2003) meaning that the 2 proteins are probably involved in cell wall metabolism during various processes and in different organs.

Our data provide evidence that $B X L 4$ is an arabinosidase as its expression in $b x / 1$ mucilage causes a significant reduction of arabinans to levels comparable with wild type (Figure 2E, Manuscript B). The overexpression of BXL4 under the TBA2 promoter in Col-0 did not induce a significant decline in arabinose levels, but there was a further reduction in xylose as compared to Col-0 (Figure 2E, Manuscript B). The nonreduction in arabinans could be explained by the BXL1 already present in Col-0 mucilage that would have already depleted all the accessible arabinans. The further 
decrease in xylose could also mean that $B X L 4$ is a more efficient xylosidase compared to $B X L 1$ when expressed in mucilage. The strong xylosidase activity of BXL4 is also evident in the bx/1 mutant complemented with $\mathrm{p} T B A 2: B X L 4$, which exhibits levels of xylose that are less than in the wild type or the control line $p T B A 2: B X L 1$ in bx/1. Work by Ralet et al., (2016) could prove the tight proportionality that occurs in xyloserhamnose-galacturonic acid of the outer layer of mucilage. Linkage analysis in this mucilage layer confirms the existence of xylan chains suggesting the presence of xylan side chains attached to rhamnogalacturonan I backbone. It is thus possible that BXL4 acts on these xylan side chains resulting in the reduction of xylose in the pTBA2:BXL4 complemented bXI1 and pTBA2:BXL4 overexpression line in Col-0.

In addition, xylans with a backbone consisting of a $\beta-1,4$-linked D-xylose are some of the hemicelluloses found in trace amounts in mucilage (Voiniciuc et al., 2015; Hu et al., 2016; Ebringerová and Heinze, 2000), and these could also serve as substrate for BXL4, as BXL1 could act on $\beta$-xylosidase substrate in vitro (Goujon et al., 2003).

Work by Goujon et al., (2003) showed that in organs, where both BXL1 and BXL4 were expressed, like in stems, flowers and siliques, betaxylosidase activity was higher, and knocking out $B X L 1$ did not cause a compensatory increase in $B X L 4$ expression. These findings hence suggested that BXL1 and BXL4 are biologically functionaly similar but not physiologically functionaly redundant. Our research findings strongly suggest that BXL4 is a bifunctional xylosidase/arabinosidase similar to BXL1. It is known that enzymes acting on polysaccharides are capable of dual functions such as Agrobacterium tumefaciens derived betaglucanases also possess betaxylosidase activity (Watt et al., 1998), whilst bacterial and fungal betaxylosidases also exhibit $\alpha$ L-arabinofuranosidase activity (Whitehead and Cotta, 2001).

Analysis of BXL4 expression by qRT-PCR in the bx/4-1 and bx/4-2 mutant lines confirmed that $b x / 4-1$ is a knockout, whilst small amounts of transcript could be detected in the bx/4-2 line (Figure 3B, Manuscript B). Both $b x / 4$ mutants exhibited a mild increase in abundance of arabinans (Figure $3 \mathrm{C}$, Manuscript $B$ ). Our data from expression of $B X L 4$ in mucilage suggests that arabinans together with xylans are a substrate of BXL4 at least in the mucilage. However, there was no noticeable increase in the xylose content of the leaf cell walls of the bx/4 mutants. Despite the increased arabinan abundance, there is no obvious morphological changes during development 
compared to wild type (Figure 3D, Manuscript B). It has already been observed that $B X L 1$ is strongly expressed in most organs including leaves, stems and siliques compared to $B X L 4$, which probably results in the non-increase of xylose in the leaves of the mutants. The betaxylosidase activity in Arabidopsis leaves is mostly attributed to BXL1 and BXL4, with the former contributing a larger portion of the activity, as the bxl1 knockout mutants have a significantly decreased betaxylosidase activity in the apoplast (Goujon et al., 2003). The bxl1 knockout mutants have a severe phenotype of smaller leaves that are curly, and shorter siliques with fewer seeds attributed to the increased xylose levels (Goujon et al., 2003). The increased abundance of arabinose due to knocking out $B X L 4$ does not cause any obvious morphological phenotype different from wild type, but it does confirm the arabinosidase activity observed after expression in mucilage.

\section{3 $B X L 4$ is a stress induced gene and could induce JA signalling}

The lack of a strong phenotype of the bx|4 mutants under optimal growth conditions is consistent with the hypothesis that BXL4 has a role especially important in stress response. Analysis of the expression of BXL4 in Arabidopsis upon mechanical wounding and $B$. cinerea infection confirmed that both forms of stress trigger the upregulation of $B X L 4$ (Figure $3 A \& D$ Manuscript B). The induction of $B X L 4$ upon wounding is similar to that of other genes involved in pectin modification. Reymond et al., (2000) showed upregulation of a pectin methylesterase and a polygalacturonase inhibitor protein upon wounding, which may help reduce further cell wall damage. OGs generated from demethylesterified pectin are usually cross-linked. It is known that demethylated OGs cross-linked by calcium ions, have a greater affinity for their receptors, wall-associated kinases, and are more biologically active in triggering defence responses (Bethke et al., 2014; Kohorn and Kohorn, 2012).

Another important stress factor that induces genes is pathogen infection. For example, the infection of Arabidopsis by $B$. cinerea results in transcriptional reprogramming of many genes. Work by Birkenbihl et al., (2012) indicated that 14 hours after infection of Arabidopsis with B. cinerea conidiospores, 1577 genes were altered in their expression levels at least 2-fold compared to mock infected plants. Our data shows that $B X L 4$ is one of the many genes that is upregulated by infection of Arabidopsis with $B$. cinerea, and work by Sham et al., (2014) is consistent with our findings because 
they also identified $B X L 4$ upregulation in their transcriptome data after $B$. cinerea infection.

Apart from an upregulation of genes that help the plants to defend against pathogens, genes that help the pathogen to invade the plant have been found to be upregulated. It is for example known that $B$. cinerea manipulates the abundance and/or activity of host proteins to aid its infection and suppress defence responses. The activation of endogenous polygalacturonases is one strategy employed by $B$. cinerea to soften the cell walls, and to aid its progression. $B$. cinerea induces the expression of endogenous polygalacturonases whose activity is not inhibited by polygalacturonase inhibitor proteins (PGIPs) as for example the tomato polygalacturonase, SLPG2A (Cervone et al., 1990). The activity of the polygalacturonase is known to soften the cell wall and to allow penetration of the fungus. AbuQamar et al., (2006), suggested that infection of Arabidopsis also resulted in the upregulation of PME3 (PECTIN METHYLESTERASE3). The activity of PME3 in plant cell walls caused the random demethylesterification of pectin, rendering it susceptible to fungal pectate lyases and polygalacturonases, whilst the mutant pme3 was less susceptible (Raiola et al., 2011). Other pathogens also employ the strategy to activate the expression of plant genes that aid their infection. The powdery mildew pathogen Erysiphe cichoracearum is known to activate the expression of PMR6 (POWDERY MILDEW RESISTANT6) which codes for a plant-derived pectate lyase-like protein known to alter the cell wall composition by degrading demethylesterified pectin. The pmr6 mutant portrays reduced susceptibility to the same pathogen (Vogel, 2002; Vogel et al., 2004). The hemibiotrophic pathogen of Arabidopsis, P. syringae pv maculicola (Psm) ES4326, is known to repress the expression of genes involved in the biosynthesis of pectin. The genes GLUCORONATE 4-EPIMERASE1 (GAE1) and GLUCORONATE 4EPIMERASE6 (GAE6) are involved in the conversion of UDP-D-glucuronic acid to UDP-D-galacturonic acid, which is a major component of pectin (Bethke et al., 2016). The gae1 gae6 double knockout mutant has reduced pectin in the cell walls and exhibits compromised resistance to $B$. cinerea and Psm. The infection of Arabidopsis with $P$. syringae pv tomato (Pst) DC3000 upregulates the expression of INFLORESCENCE DEFICIENT IN ABSCISSION-like 6 (IDL6) (Wang et al., 2017). IDL6 triggers the expression of an endogenous polygalacturonase ADPG2, which normally degrades pectin during abscission of floral organs (Estornell et al., 2015). 
The induced expression of IDL6 resulted in an increased susceptibility to Pst DC3000, whilst the idl6 knockout mutants portrayed greater resistance to the bacterial pathogen (Wang et al., 2017).

On the other hand, plant infection with $B$. cinerea induces the upregulation of genes that are involved in defences against the pathogen. Infection of plants with various pathogens results in the upregulation of polygalacturonase inhibitor proteins (PGIPs) (Yao et al., 1999). PGIPs are important defence proteins belonging to a family of Leurich repeat (LRR) proteins and are present in cell walls of most plant species (Toubart et al., 1992; Ferrari, 2003). In Arabidopsis, infection with B. cinerea triggers the upregulation of two tandemly duplicated PGIPs via two signal transduction pathways. JA-lle mediates the expression of AtPGIP2, whilst AtPGIP1 expression is mediated by oligogalacturonides. Overexpression of the two genes results in reduced disease symptoms after infection with B. cinerea (Ferrari, 2003). Inhibition of polygalacturonases by PGIPs facilitates the generation of oligogalacturonides with a high degree of polymerisation that elicit various defence responses (Cervone et al., 1989). In beans infected with the pathogen Colletotrichum lindemuthianum, the transcripts of PGIP accumulate causing a correlated increase in hypersensitive response in incompatible interactions. In the event of a compatible interaction, there is a delay in PGIP transcript accumulation that also coincides with appearance of disease symptoms, thus emphasising the role of PGIP expression in resistance to fungal pathogens (Nuss et al., 1996). In sugar beet, a pectin lyase inhibitor that suppresses the activity of a Rhizoctonia solani pectin lyase is upregulated upon infection with the pathogen. Plant resistance to the pathogen is correlated to the expression levels of the inhibitor protein (Bugbee, 1993).

The pectin methylesterase inhibitor protein GhPMEl3 found in cotton (Gossypium hirsutum) is unpregulated after infection with the fungus Verticillium dahliae. It was found out that GhPMEI3 increases pectin methylesterification through the inhibition of cottons PME2 and PME31 and this reduced fungal development in cotton. Silencing of GhPMEl1 caused an increased susceptibility to the fungal pathogen (Liu et al., 2018). In pepper (Capsicum annuum L.), CaPMEl1 is upregulated upon infection with Xanthomonas campestris pv vesicatoria $(X c v)$. PMEl1 inhibits the activity of PMEs and also exhibits antifungal activity against some phytopathogenic fungi. The silencing of 
CaPMEl1 in pepper resulted in increased susceptibility to $X c v$ together with the reduced expression of some plant defence genes (Liu et al., 2018).

Our gene expression data showed that $B X L 4$ was a stress induced gene upregulated upon mechanical wounding and attack from the necrotrophic pathogen $B$. cinerea. Both forms of stress are known to trigger JA-lle signalling which inturn induces the expression of various defence responses (Pangesti et al., 2016; Pieterse et al., 2012). To check if JA-lle was responsible for upregulation of $B X L 4$ we investigated $B X L 4$ expression in wild type Arabidopsis versus a JA-lle deficient mutant delayeddehiscence2-2 (dde2-2) (von Malek et al., 2002) after mechanical wounding. Wounding in Arabidopsis is known to induce various genes to restrict further damage (Reymond and Farmer, 1998) and the expression of many of these genes is JA regulated (Farmer et al., 1998; Creelman and Mullet, 1997). The production of JA and its active derivative JA-lle resulting in activation of their downstream signalling pathway is critical for plant defence against wounding and attack from necrotrophic pathogens (Yan and Xie, 2015; Howe and Jander, 2008). Pattern recognition receptors (PRRs) located on the plasma membrane detect danger cues such as PAMPs, herbivore associated molecular patterns (HAMPs) and DAMPs, triggering a rapid accumulation of JA-lle (Lee and Howe, 2003; Schmelz, 2003; Geng et al., 2014). The dde2-2 mutant has a mutation in ALLENE OXIDASE SYNTHASE (AOS). AOS carries out the dehydration of 13-hydroperoxy-octadecatrienoic acid forming an unstable epoxide that is catalysed to 12-oxo-phytodienoic acid (OPDA) by ALLENE OXIDE CYCLASE (AOC) during early stages of JA biosynthesis (Figure 8) (Turner et al., 2002). Wounding of the dde2-2 did not induce the upregulation of $B X L 4$ (Figure $4 A$, Manuscript $B$ ). This finding suggests that BXL4 is also one of the genes whose expression in response to wounding requires JA-lle signalling suggesting a putative role in plant defences.

Because the $b x / 4$ mutants had an increased amount of arabinans, we further checked if this cell wall modification had any effect on plant defence signalling by measuring the accumulation of defence marker genes after wounding and $B$. cinerea infection. The bx/4 mutant plants had reduced expression of JA-lle marker genes JAZ10 and $P D F 1.2$ as well as the reduction in the accumulation of JA and JA-lle after wounding and $B$. cinerea infection. The reduction in JA-lle production after wounding and $B$. cinerea infection suggests that BXL4 is not only upregulated by JA-lle but plays a role 
in the amplification of JA-lle signalling as well. Pectin-derived fragments have been implicated in the regulation of enzymes involved in the biosynthetic pathway of JA-lle. Denoux et al., (2008), demonstrated the participation of oligogalacturonides in the activation of two lipoxygenases LOX3 and LOX4 that take part in the biosynthetic pathway of JA-lle. The treatment of Arabidopsis with short oligosaccharides also induces the upregulation of another gene, AOS, involved in JA-lle biosynthesis (Norman et al., 1999). The possible involvement of oligosaccharides in JA-lle signalling comes also from less direct evidence. CYP79B2, CYP79B3 and CYP83B1 are upregulated upon treatment of Arabidopsis with oligogalacturonides. These genes are players in the biosynthesis of indole glucosinolates (Wittstock and Halkier, 2002) and JA regulates the accumulation of indole glucosinolates after infection of Arabidopsis with Erwinia carotovora (Brader, 2001). Many other publications have indicated that alterations in cell wall composition caused by different mutations induce the reprogramming of various phytohormone signalling pathways (Vega-Sanchez et al., 2012; Wang et al., 2017; Ellis, 2002). It is also known that JA-lle induces the expression of its biosynthetic genes, which in turn trigger JA-lle accumulation through a positive feedback loop hence resulting in a robust defence response (Browse, 2009). Observations from our wounding and $B$. cinerea infection data suggests that $B X L 4$ is induced in a JA-lle dependant manner, and contributes to induction of JA-lle accumulation in a positive feedback mechanism, indicating a possible role in plant defences. 


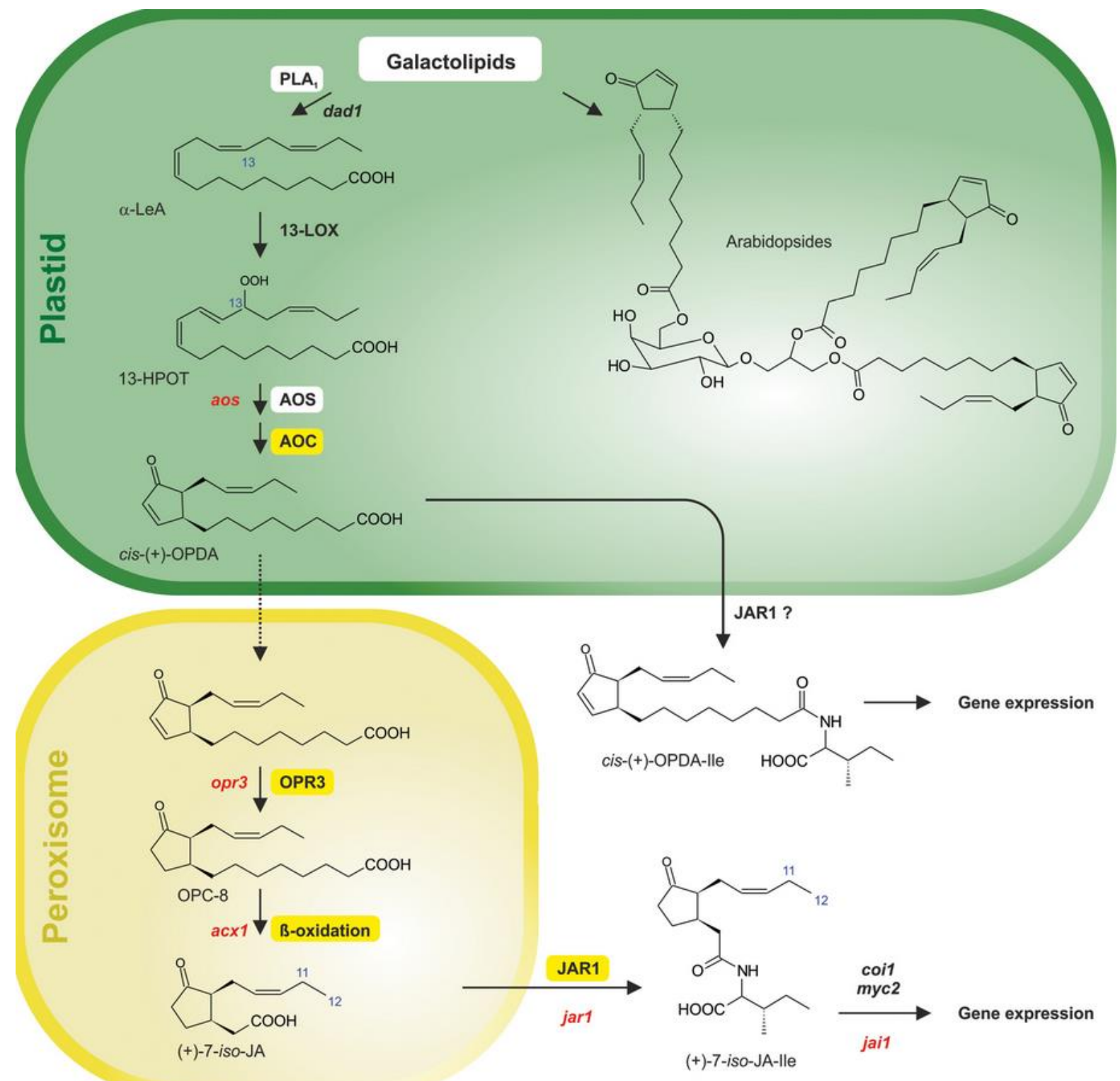

Figure 8: The biosynthesis of JA: Image adapted from Wasternack and Song, (2016) Phytopathogen infection, feeding by herbivorous insects or mechanical damage, causes the release of $\alpha$-linolenic acid from membranes of chloroplasts. $\alpha$ linolenic acid is converted to 12-oxo-phytodiecanoic acid (OPDA) through a series of enzyme steps involving LIPOXYGENASE (LOX), followed by an ALLENE OXIDE SYNTHASE (AOS) which forms an unstable product that is quickly converted by ALLENE OXIDE CYCLASE (AOS). After transportation to the peroxisome, OPDA then undergoes several B-oxidation cycles to form (+)-7-iso-JA (Zhang et al., 2017; Larrieu and Vernoux, 2016). (+)-7-iso-JA is then transported to the cytosol upon which the amino acid isoleucine (Ile) is conjugated to form JA-lle, which is the most active JA derivative.

Analysis of defence marker genes after $B$. cinerea infection also showed that another defence related gene PAD3 had lower expression in the bx/4 mutants compared to wild type lines. The accumulation of the phytoalexin camalexin was reduced in the 
mutant lines after B. cinerea infection. PAD3 is a cytochrome P450 responsible for catalysing the last step in the camalexin biosynthesis pathway (Zhou, 1999). PAD3 is induced upon perception of oligogalacturonides by membrane bound WALL ASSOCIATED KINASE1 (WAK1), which activates downstream MAPK cascades (Ferrari, 2013). Camalexin is the most abundant phytoalexin in Arabidopsis and its biosynthesis is triggered in part by the perception of MAMPs and also oligogalacturonides (Ferrari et al., 2007). Camalexin biosynthesis is also regulated by the JA-lle signalling pathway. Rowe et al., (2010) indicated that the accumulation of camalexin after $B$. cinerea infection in Arabidopsis mutants deficient in JA-lle signalling or JA-lle perception was significantly reduced compared to wild type lines. It is therefore possible that the reduction in JA-lle biosynthesis observed in the bx/4 mutants could be responsible for the reduced production of camalexin. Observations from out data indicates that the expression of $P A D 3$ in the $b x / 4$ mutant was significantly reduced, but there was no similar reduction in camalexin accumulation, where a difference was only observed after 72 hours. Whilst $P A D 3$ is responsible for catalysing the last step in camalexin biosynthesis, overexpression of PAD3 alone does not necessarily result in camalexin accumulation, and that all genes in the camalexin biosynthesis pathway have to be elicited (Schuhegger et al., 2006).

The reduction in expression of JA-lle marker genes and PAD3 has been linked to oligosaccharides that are derived from homogalacturonan degradation (Ferrari, 2013) and not rhamnogalacturonan which BXL4 probably acts on. It has however, been demonstrated that RG-I itself and therefore RG-I-modifying proteins have an effect on the nature of homogalacturonan and consequently on the nature of the derived oligogalacturonans. The oligogalacturonide-derived responses described above in relation to JA-lle and camalexin could also be indirectly affected by RG-I modifications induced by BXL4. RG-I side chain modifications affect the degree of methylesterification of homogalacturonan. Western et al., (2001), could show that the mum2 mutant, characterised by the presence of abundant galactan side chains in mucilage RG-I, has homogalacturonan with a high degree of methylesterification. This is evidence that PME activity is altered in response to RG-I modification. Similarly, Arabidopsis plants carrying a mutation in the transcriptional regulator LEUNIG_HOMOLOG (LUH) produce mucilage with highly substituted RG-I (Walker et al., 2011). The luh mutant has a high abundance of galactan and arabinan chains 
attached to the RG-I backbone. Homogalacturonan from this mutant also shows a high degree of methylesterification similar to what is observed in mum2. The increased arabinan content of RG-I associated with bx/4 could play a similar role in inhibiting PME activity resulting in generation of methylesterified oligogalacturonans, which are known to have a low elicitor activity. Partially demethylesterified oligogalacturonans have been found to induce strong biological responses in strawberries, triggering the induction of lipoxygenases, and increasing resistance to B. cinerea (Osorio et al., 2008).

The predicted interaction partners of BXL4 generated using STRING version 11.0 (Szklarczyk et al., 2015a) implicate the possible role of BXL4 in the generation of oligogalacturonides (Figure 9). The prediction indicates that some polygalacturonases and pectin lyases are potential interactors. The polygalacturonase PGA4 and threepectin lyase like proteins AT3G59870, AT5G14650 and AT3G07850 are indicated as potential interaction partners which act on homogalacturonan and might work cooperatively with BXL4 contributing in generation of oligogalacturonides with elicitor activity.

Whilst we have indicated the activity of BXL4 to be indirectly linked to defence responses by affecting the nature of homogalacturonan and its oligogalacturonides. It is also possible that the direct modifications possibly done on RG-I will have plant defence implications. The activity of BXL4 cleaves off arabinose monomers during the remodelling of cell wall polysaccharides which are unlikely to have any elicitor activity. However, it is possible that the modified RG-I left could trigger some biological effects. An interesting observation was made by Breitenbach et al., (2014), who performed comparative proteomics analysis of apoplast-enriched proteins extracts from enhanced disease susceptibility 1 (eds1) mutants and wild type Arabidopsis expressing AvrRpm1 an effector from $P$ syringae. They identified BXL4 as one of the EDS1 dependent proteins upregulated in the apoplast upon expression of a pathogen effector, thus suggesting its possible role in plant defences. They also identified a LEGUME LECTIN LIKE PROTEIN1 (LLP1) on the plasma membrane facing the apoplast as another EDS1 dependant protein. It is thus, possible that these two proteins that are simultaneously upregulated are interactors, and modified RG-I could bind to LLP1 and trigger defence responses. In mammalian cells, lectins are known to recognise non-self, self or modified self-molecular components and activation of 
trafficking in the cells together with communication between cells leading to immune signalling (Rabinovich et al., 2012). LLP1 may regulate the plant defences after recognition of the modified RG-I either in the locally $B$. cinerea infected leaf or systemic leaves leading to induction of plant defence responses.

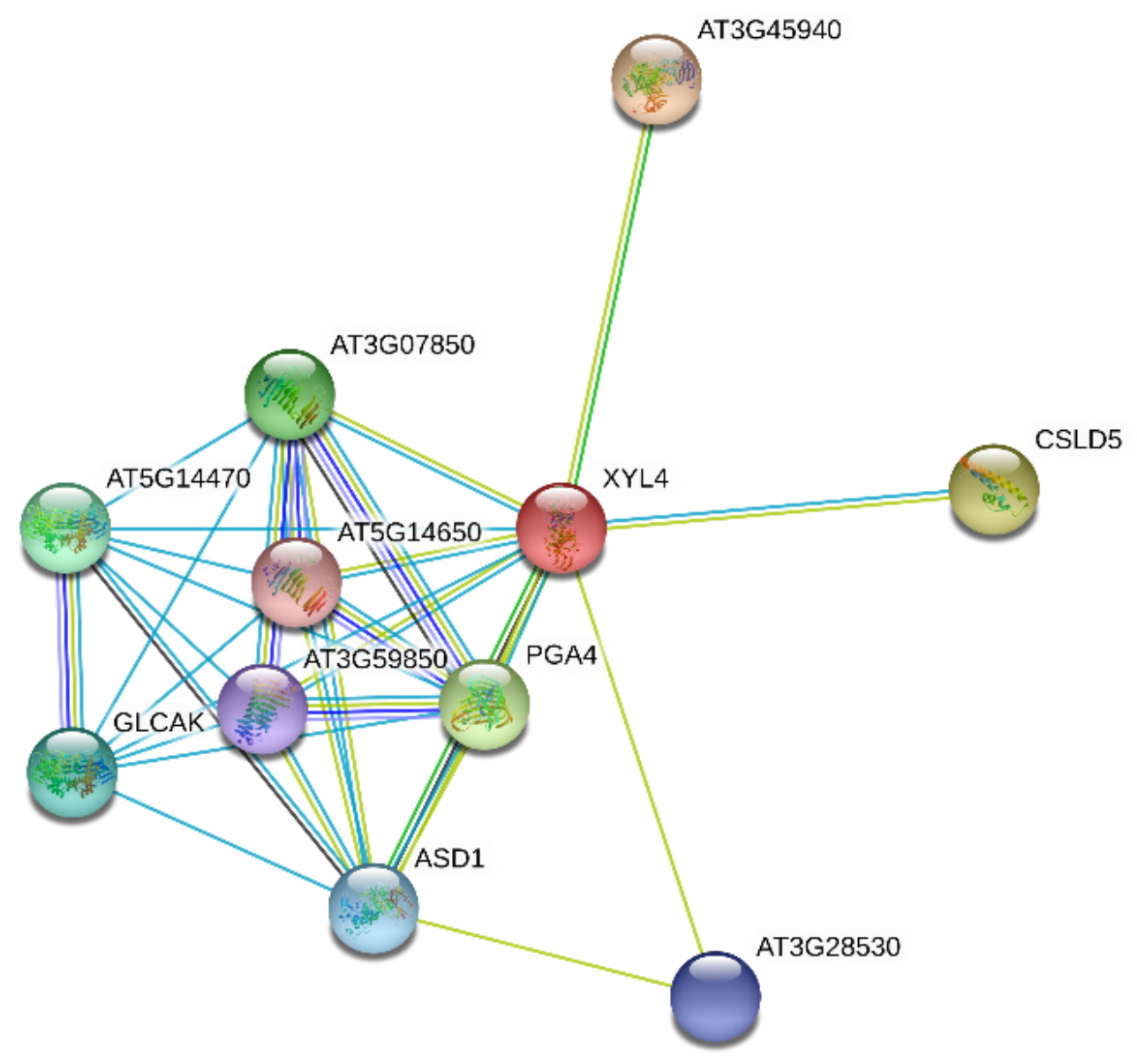

Known Interactions

C-O from curated databases

C-O experimentally determined
Predicted Interactions

C-O gene neighborhood

C-O gene fusions

C-O gene co-occurrence
Others

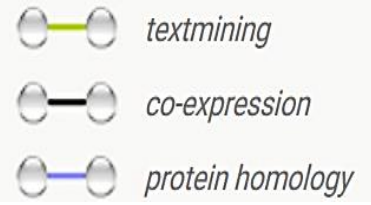

Figure 9: Predicted BXL4 functional partners. Image generated using STRING version $11.0 \mathrm{https}$ ://string-db.org (Szklarczyk et al., 2015a). 
Table 2: Predicted functional partners of BXL4 shown in figure 9.

\begin{tabular}{|l|l|}
\hline AT3G45940 & Glycosyl hydrolase family 31 protein \\
\hline CSLD5 & Cellulose synthase like protein D5 \\
\hline PGA4 & Exopolygalacturonase clone GBGE184 \\
\hline AT3G07850 & Pectin lyase like superfamily protein \\
\hline AT5G14470 & GHMP kinase family protein; Sugar-1-kinase \\
\hline GLCAK & Glucuronokinase 1; Sugar-1-kinase \\
\hline ASD1 & Alpha-L-arabinofuranosidase 1 \\
\hline AT3G28530 & UDP-glucose 4-epimerases \\
\hline AT3G59850 & Pectin lyase like superfamily protein \\
\hline AT5G14650 & Pectin lyase like super family protein \\
\hline
\end{tabular}

\subsection{BXL4 contributes to resistance against $B$. cinerea}

In order to investigate the significance of $B X L 4$ induction after mechanical wounding and $B$. cinerea infection to plant immunity, a disease susceptibility assay was carried out. The bx/4 mutant lines were significantly less resistant to B.cinerea compared to wild type plants. Data from our infection assays provide evidence that $B X L 4$ is a player contributing to resistance against the necrotrophic pathogen $B$. cinerea.

Findings from our work indicate that $B X L 4$ contributes to plant immunity possibly through various mechanisms including the reduction in JA production. Mechanical wounding of Arabidopsis leaves and infection with $B$. cinerea resulted in reduced expression of JA-lle marker genes in the bx/4 knockout lines compared to the wild type. The same observation was seen in terms of JA and JA-lle accumulation post wounding or infection. Wounding of plant tissues together with infection of Arabidopsis with necrotrophic pathogens is known to trigger JA-lle signalling as a defence response (Browse and Howe, 2008). The outcome of plant-pathogen interactions is determined to a large extend by the hormonal homeostasis in plants. While it is generally agreed that salicylic acid $(S A)$ is responsible for resistance to biotrophic pathogens and JA-lle for resistance to necrotrophs (Rahman et al., 2012), there is an overlap and cross talk between the phytohormones impacting on the various defence responses (Kachroo, 2003; Veronese, 2006). The role of JA-lle with regards to resistance to necrotrophs has been well documented in many articles, and generally, treatment of plants with JA-lle renders these more resistant to necrotrophic pathogens 
whilst knocking out genes involved in JA-lle biosynthesis results in increased susceptibility (Vijayan et al., 1998; Lorenzo and Solano, 2005). CORONATINE INSENSITIVE1 (COI1) is a receptor of JA-lle in Arabidopsis, and mutations in this gene lead to JA-lle insensitivity thereby increasing susceptibility to many necrotrophic pathogens including $B$. cinerea (Thomma et al., 1998).

Jasmonates trigger the expression of their own biosynthetic genes causing a positive feedback loop that promotes JA-lle accumulation (Browse, 2009). Jasmonates regulate the expression of cell wall proteins important for stress response. Taurino et al., (2014), showed that potato (Solanum tuberosum) transgenic plants lacking both St13-ALLENE OXIDE SYNTHASE genes required for JA-lle biosynthesis had altered cell wall pectin composition compared to wild type plants. The transgenic plant named CoAOS1/2 had a PME activity that was different from wild type, as the pectin of this mutant was highly methylated. They also indicated that the transgenic lines produced less amounts of a rhamnogalacturonan-derived oligosaccharide and were more susceptible to the necrotrophic fungal pathogen Dickeya dadantii. JA-lle is known to upregulate the expression of $P G I P 2$, which contributes to cell wall rigidity by inhibiting the activity of polygalacturonases derived from necrotrophic pathogens (O'Brien et al., 2012; Bellincampi et al., 2014).

The bx/4 mutants had a reduction in the expression of $P A D 3$ and accumulation of camalexin after infection with $B$. cinerea compared to wild type plants. Camalexin is an indole derivative of tryptophan forming part of the defence repertoire induced in plants upon perception of various cues and in response to both necrotrophic and biotrophic pathogens (Ahuja et al., 2012). Arabidopsis mutants impaired in biosynthesis of camalexin were found to be more susceptible to a variety of phytopathogens including B. cinerea (Denby et al., 2004). Whilst the antimicrobial activity of camalexin has been confirmed in vitro, the exact mechanism involved is not very clear (Pedras and Khan, 2000; Stotz et al., 2011). In B. cinerea however, exposure to camalexin leads to a fungal programmed cell death-like response (Shlezinger et al., 2011). Hence, the production of camalexin in plant tissues upon B. cinerea infection could lead to this programmed cell death, limiting further development of the pathogen. In bacterial pathogens, camalexin is known to disrupt their cell membranes, and this probably explains why the Psm strain ES4326 is sensitive to camalexin treatment (Rogers et al., 1996). Alternaria brassicicola treated 
with camalexin exhibits a transcriptional profiling skewed towards plasma membrane maintenance and reduced permeability of the cell wall, thus indicating the potential effects of camalexin on membranes (Sellam et al., 2007).

The bx/4-1 mutants also exhibited mildly higher arabinan levels in the leaf cell walls compared to wild type plants. The difference is not dramatic but given that the betaxylosidase family has seven members, redundancy cannot be ruled out. The necrotrophic pathogen $B$. cinerea is known to prefer a host range that includes pectinrich species (ten Have et al., 2002). The production of cell wall degrading proteins during infection helps in hydrolysis of the pectin component of the cell wall into its monomeric units, thus providing a source of carbon to the growing pathogen (Zhang and van Kan, 2013). The vast cell wall degrading enzyme catalogue including arabinanases (Nafisi et al., 2014) secreted by B. cinerea could also suggest that arabinans can also be degraded as a carbon source. It is known that degradation of cell wall components during pathogen infection, especially by $B$. cinerea, aids in the rapid development and spread of the pathogen (Battaglia et al., 2011; Zhang et al., 2011). The increased abundance of arabinans in the bx/4 mutants could serve as an extra source of carbon for $B$. cinerea resulting in the increased disease development observed compared to the wild type.

The altered cell wall composition could also have an effect on the hyphal penetration of $B$. cinerea. Whilst $B$. cinerea invades plant tissues using a host of various cell wall degrading enzymes, it is known that softening of the cell walls through induction of endogenous polygalacturonases is important for virulence. The induction of endogenous polygalacturonases, which results in generation of oligogalacturonides that do not trigger defence responses but allow softening of cell walls (Kohorn and Kohorn, 2012), helps in fungal hyphae development. Arabinans are known to play a role in cell wall stiffening, which might impede the development of hyphae. The role of arabinans in cell wall stiffening has been articulated in various publications. For instance, the arad1 mutant, with reduction in cell wall arabinans, has an inflorescence stem more rigid than the wild type. Jones et al., $(2003 ; 2005)$ showed that arabinans are important for promoting guard cell flexibility. The mechanism in which arabinans affect cell wall stiffening is not clear, but it is thought that they hinder the close association of homogalacturonans, hence reducing the formation of calcium crosslinks that promote cell wall rigidity. The possible role of arabinans in restricting the 
formation of calcium cross-links could also aid in disease susceptibility observed. The generation of calcium cross-linked oligogalacturonides after cell wall hydrolysis triggers a strong defence response. Decreux and Messiaen, (2005) proved that WAK1 has a stronger affinity for calcium cross-linked oligogalacturonides triggering a stronger biological response. The contribution of arabinans to cell wall stiffness that inhibits fungal penetration is shown in the mur8-1 mutant, which shows a reduction in cell wall RG-I (Mertz et al., 2012). Engelsdorf et al., (2017) could prove that the mur81 mutant has a compromised resistance to the penetration of the fungal pathogen Colletotrichum higginsianum.

The possible role of BXL4 in cell wall stiffening is also suggested by its expression pattern in Arabidopsis rosette leaves. Our data shows that the expression of BXL4 was mostly confined to mature fully developed leaves, and lower in the younger developing leaves. The fully developed leaves do not need to expand in contrast to the younger leaves, thus, they become rigid. The contribution of RG-I to cell wall stiffening is not only confined to the arabinans, but also the galactan side chains have been implicated in cell wall stiffening. Martín et al., (2011) highlighted that the chickpea betagalactosidase $\mathrm{BI}$-Gal that cleaves off RG-I galactan side chain was important for modification of cell wall polymers and results in thickened cell walls. Degradation of 1,4 galactans has also been shown to induce the reinforcement of phloem cell walls (Vincken et al., 2003). Another galactosidase was shown to associate with vascular tissues of Cicer arietinum after the cells had ceased elongation and expansion, thus emphasising its role in cell wall fortification (Martín et al., 2008).

The trimming of RG-I side chains is an important pre-requisite for hydrolysis of both RG-I and homogalacturonan by rhamnogalacturonases and polygalacturonases, respectively (Mutter et al., 1998). The increased abundance of arabinan side chains in the mutant lines could hinder the activity of polygalacturonases that degrade HG and thereby generate elicitor active oligosaccharides. Figure 9 shows that $B X L 4$ is possibly linked to various proteins involved in the degradation of pectin based on STRING analysis (Szklarczyk et al., 2015b). A polygalacturonase (PGA4), three pectin lyase-like proteins AT3G59870, AT5G14650 and AT3G07850 and an alpha-Larabinofuranosidase ASD1 are predicted functional partners of BXL4, which may act downstream, resulting in the generation of oligosaccharides required for defence responses. An overabundance of arabinans may result in increased molecular 
entanglement on cell wall polysaccharides leading to reduced activity of glycosyl hydrolases acting downstream of BXL4. The synergistic activity of arabinofuranosidases and $\beta$-galactosidases to trim side chains of RG-I is an early step important for depolymerisation of pectin by polygalacturonases (Yoshioka et al., 1995).

In summary the possible contribution of BXL4 to plant defences is illustrated in the model below (Figure10). 


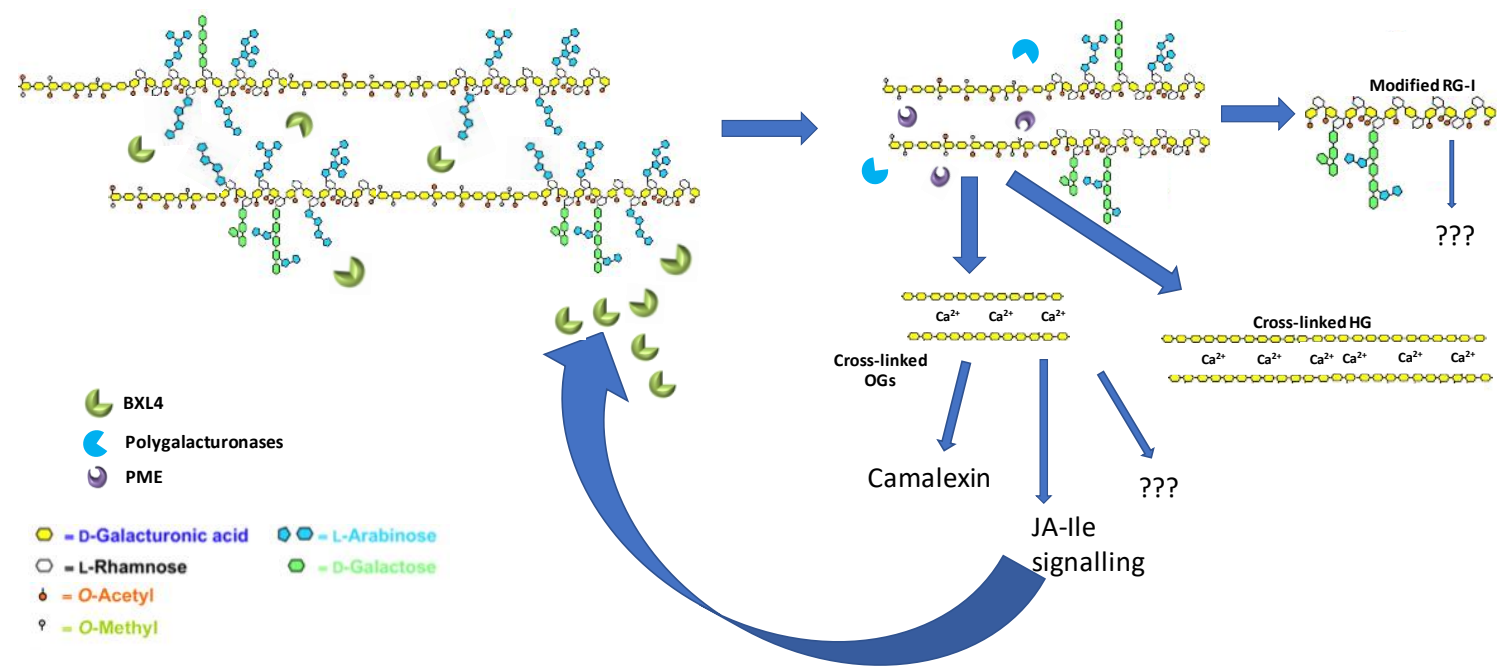

Figure 10: Predicted model showing role of BXL4 in plant defences. Plant cell wall damage due to wounding and pathogen attack triggers the induction of BXL4 in a JA-Ile dependant manner. BXL4 trims arabinans present in the apoplast (possibly RGI side chain arabinans). The activity of BXL4 reduces the molecular entanglement in the apoplast and allows more efficient activity of PMEs which demethylesterify the homogalacturonan domains allowing for the cross-linkage of homogalacturonan with $\mathrm{Ca}^{2+}$. Cross-linked homogalacturonan is rigid and limits the advancement of fungal hyphae. Polygalacturonase activity linked to BXL4 activity results in the generation of demethylesterified cross-linked oligogalacturonides, which elicit strong biological responses leading to the induction of JA-lle and camalexin biosynthetic genes and the resultant accumulation of JA-Ile and camalexin. Ja-lle signalling leads to the induction of JA related genes including BXL4 in a feedback loop, and consequently a stronger response reaction.

\section{Conclusion}

The remodelling of cell wall polysaccharides upon infection with various pathogens is still an underexplored field mainly due to the complexity associated with cell wall analytics. This project sought to contribute to the development of a robust and convenient method to use in analytics of cell wall derived from different sources. We managed to develop a GC-MS based method for analysing water extracted pectin from various tissue including mucilage, leaf cells and commercial pectin. Using our GC-MS method, we could determine the compositions of various pectin extracts to levels similar to the ones previously published. The method could detect the increased abundance of arabinose in the mucilage of $b x / 1$ mutant compared to wild type plants. The method offers several advantages including being able to detect all the pectic 
sugar, both neutral sugars and galacturonic acid in one reaction cycle. The use of TFA employed in the protocol saves time compared to acid methanolysis. The method allows for the correct identification of monosaccharides, as it does not only rely on retention times but also uses MS-based detection.

Furthermore, we could characterise the enzyme activity of BXL4 through its expression in seed coat epidermal cells and analysis of cell walls of bx/4 mutants. $\mathrm{BXL} 4$ is able to rescue the mucilage extrusion phenotype, which is altered in the $b x / 1$ mutant. We could observe a decrease in arabinose levels in the mucilage of $b x / 1$ mutant complemented with BXL4 to levels comparable to the wild type. BXL4 overexpression lines in Col-0 showed a decreased abundance of xylose compared to wild type thus confirming a bifunctional role of BXL4 as it acts as both an arabinosidase and xylosidase. The bx/4 mutants also have a mild increase in arabinose levels, but the overall growth is not affected.

BXL4 was also shown to be a stress related gene, as both mechanical wounding of leaves and $B$. cinerea infection induced the upregulation of the gene. However, the gene is not upregulated after wounding of the JA-lle deficient mutant dde2-2, whilst the $b x / 4$ mutants also do not have induction of JA-lle marker genes upon mechanical wounding or $B$. cinerea infection. We therefore speculate that BXL4 is a JA-Ile dependant gene, which in a positive feedback induces the upregulation of JA-Ile marker gene, a phenomenon that occurs with JA-lle biosynthetic genes. B. cinerea infection of the bx/4 mutants results in a reduced accumulation of JA-lle and camalexin in comparison to wild type plants. Infection of Arabidopsis plants with $B$. cinerea indicates a more susceptible phenotype in the bx/4 mutants compared to wild type plants. We thus speculate that BXL4 is a stress induced gene that is regulated by JAIle signalling and is important for RG-I remodelling as a mitigatory response to pathogen infection. The pathogen defence pathway of BXL4 involves both JA-lle signalling and camalexin production. 


\section{References}

AbuQamar, S., Chen, X., Dhawan, R., Bluhm, B., Salmeron, J., Lam, S., Dietrich, R.A., and Mengiste, T. (2006). Expression profiling and mutant analysis reveals complex regulatory networks involved in Arabidopsis response to Botrytis infection. Plant J. 48: 28-44.

Agüero, C.B., Uratsu, S.L., Greve, C., Powell, A.L.T., Labavitch, J.M., Meredith, C.P., and Dandekar, A.M. (2005). Evaluation of tolerance to Pierce's disease and Botrytis in transgenic plants of Vitis vinifera L. expressing the pear PGIP gene. Mol. Plant Pathol. 6: 43-51.

Ahuja, I., Kissen, R., and Bones, A.M. (2012). Phytoalexins in defense against pathogens. Trends Plant Sci. 17: 73-90.

Albenne, C., Canut, H., and Jamet, E. (2013). Plant cell wall proteomics: the leadership of Arabidopsis thaliana. Front. Plant Sci. 4.

Almagro Armenteros, J.J., Tsirigos, K.D., Sønderby, C.K., Petersen, T.N., Winther, O., Brunak, S., von Heijne, G., and Nielsen, H. (2019). SignalP 5.0 improves signal peptide predictions using deep neural networks. Nat. Biotechnol. 37: 420-423.

Arsovski, A.A., Haughn, G.W., and Western, T.L. (2010). Seed coat mucilage cells of Arabidopsis thaliana as a model for plant cell wall research. Plant Signal. Behav. 5: 796-801.

Arsovski, A.A., Popma, T.M., Haughn, G.W., Carpita, N.C., McCann, M.C., and Western, T.L. (2009). AtBXL1 encodes a bifunctional beta-D-xylosidase/alpha$\mathrm{L}$-arabinofuranosidase required for pectic arabinan modification in Arabidopsis mucilage secretory cells. Plant Physiol. 150: 1219-34.

Atmodjo, M.A., Hao, Z., and Mohnen, D. (2013). Evolving views of pectin biosynthesis. Annu. Rev. Plant Biol. 64: 747-79.

Aziz, A., Gauthier, A., Bezier, A., Poinssot, B., Joubert, J.-M., Pugin, A., Heyraud, A., and Baillieul, F. (2007). Elicitor and resistance-inducing activities of $-1,4$ cellodextrins in grapevine, comparison with $-1,3$ glucans and $-1,4$ 
oligogalacturonides. J. Exp. Bot. 58: 1463-1472.

Babu, Y. and Bayer, M. (2014). Plant Polygalacturonases Involved in Cell Elongation and Separation-The Same but Different? Plants (Basel, Switzerland) 3: 613-23.

Bacete, L., Mélida, H., Miedes, E., and Molina, A. (2018). Plant cell wall-mediated immunity: cell wall changes trigger disease resistance responses. Plant J. 93: 614-636.

Backhouse, D. and Willetts, H.J. (1984). A histochemical study of sclerotia of Botrytis cinerea and Botrytis fabae. Can. J. Microbiol. 30: 171-178.

Battaglia, E., Benoit, I., van den Brink, J., Wiebenga, A., Coutinho, P.M., Henrissat, B., and de Vries, R.P. (2011). Carbohydrate-active enzymes from the zygomycete fungus Rhizopus oryzae: a highly specialized approach to carbohydrate degradation depicted at genome level. BMC Genomics 12: 38.

Baydoun, E.A.-H., Rizk, S.E., and Brett, C.T. (1999). Localisation of Meth Itransferases Involved in Glucuronoxylan and Pectin Methylation in the Golgi Apparatus in Etiolated Pea Epicotyls. J. Plant Physiol. 155: 240-244.

Bellincampi, D., Cervone, F., and Lionetti, V. (2014). Plant cell wall dynamics and wall-related susceptibility in plant-pathogen interactions. Front. Plant Sci. 5.

Bellincampi, D., Dipierro, N., Salvi, G., Cervone, F., and De Lorenzo, G. (2000). Extracellular $\mathrm{H} 2 \mathrm{O} 2$ Induced by Oligogalacturonides is Not Involved in the Inhibition of the Auxin-Regulated rolB Gene Expression in Tobacco Leaf Explants. Plant Physiol. 122: 1379-1386.

Benedetti, M., Verrascina, I., Pontiggia, D., Locci, F., Mattei, B., De Lorenzo, G., and Cervone, F. (2018). Four Arabidopsis berberine bridge enzyme-like proteins are specific oxidases that inactivate the elicitor-active oligogalacturonides. Plant J. 94: 260-273.

Bethke, G. and Glazebrook, J. (2019). Measuring Pectin Properties to Track Cell Wall Alterations During Plant-Pathogen Interactions. Methods Mol. Biol. 1991: $55-60$ 
Bethke, G., Grundman, R.E., Sreekanta, S., Truman, W., Katagiri, F., and Glazebrook, J. (2014). Arabidopsis PECTIN METHYLESTERASEs contribute to immunity against Pseudomonas syringae. Plant Physiol. 164: 1093-107.

Bethke, G., Thao, A., Xiong, G., Li, B., Soltis, N.E., Hatsugai, N., Hillmer, R.A., Katagiri, F., Kliebenstein, D.J., Pauly, M., and Glazebrook, J. (2016). Pectin Biosynthesis Is Critical for Cell Wall Integrity and Immunity in Arabidopsis thaliana. Plant Cell 28: 537-56.

Bindschedler, L. V, Dewdney, J., Blee, K.A., Stone, J.M., Asai, T., Plotnikov, J., Denoux, C., Hayes, T., Gerrish, C., Davies, D.R., Ausubel, F.M., and Bolwell, G.P. (2006). Peroxidase-dependent apoplastic oxidative burst in Arabidopsis required for pathogen resistance. Plant J. 47: 851-63.

Birkenbihl, R.P., Diezel, C., and Somssich, I.E. (2012). Arabidopsis WRKY33 Is a Key Transcriptional Regulator of Hormonal and Metabolic Responses toward Botrytis cinerea Infection. Plant Physiol. 159: 266-285.

Biswal, A.K., Tan, L., Atmodjo, M.A., DeMartini, J., Gelineo-Albersheim, I., Hunt, K., Black, I.M., Mohanty, S.S., Ryno, D., Wyman, C.E., and Mohnen, D. (2017). Comparison of four glycosyl residue composition methods for effectiveness in detecting sugars from cell walls of dicot and grass tissues. Biotechnol. Biofuels 10.

Bochicchio, R. and Reicher, F. (2003). Are hemicelluloses from Podocarpus lambertii typical of gymnosperms? Carbohydr. Polym. 53: 127-136.

Boller, T. and Felix, G. (2009). A renaissance of elicitors: perception of microbeassociated molecular patterns and danger signals by pattern-recognition receptors. Annu. Rev. Plant Biol. 60: 379-406.

Bosch, M. (2005). Pectin Methylesterase, a Regulator of Pollen Tube Growth. Plant Physiol. 138: 1334-1346.

Bourlard, T; Pellerin, P; Morvan, C. (1997). Rhamnogalacturonan I and II are pectic substrates for flax-cell methyltransferases. Plant Physiol. Biochem.: 623629. 
Bouton, S., Leboeuf, E., Mouille, G., Leydecker, M.-T., Talbotec, J., Granier, F., Lahaye, M., Höfte, H., and Truong, H.-N. (2002). QUASIMODO1 encodes a putative membrane-bound glycosyltransferase required for normal pectin synthesis and cell adhesion in Arabidopsis. Plant Cell 14: 2577-90.

Brader, G. (2001). Jasmonate-Dependent Induction of Indole Glucosinolates in Arabidopsis by Culture Filtrates of the Nonspecific Pathogen Erwinia carotovora. Plant Physiol. 126: 849-860.

Brandhoff, B., Simon, A., Dornieden, A., and Schumacher, J. (2017). Regulation of conidiation in Botrytis cinerea involves the light-responsive transcriptional regulators BcLTF3 and BcREG1. Curr. Genet. 63: 931-949.

Breitenbach, H.H., Wenig, M., Wittek, F., Jordá, L., Maldonado-Alconada, A.M., Sarioglu, H., Colby, T., Knappe, C., Bichlmeier, M., Pabst, E. and Mackey, D. (2014). Contrasting Roles of the Apoplastic Aspartyl Protease APOPLASTIC, ENHANCED DISEASE SUSCEPTIBILITY1-DEPENDENT1 and LEGUME LECTIN-LIKE PROTEIN1 in Arabidopsis Systemic Acquired Resistance,. Plant Physiol. 165: 791-809.

Brickell, L.S. and Grant Reid, J.. (1996). Biosynthesis in vitro of pectic $(1 \rightarrow 4)-\beta-D-$ galactan. In, pp. 127-134.

Broekaert, W.F. and Peumans, W.J. (1988). Pectic polysaccharides elicit chitinase accumulation in tobacco. Physiol. Plant. 74: 740-744.

Brown, R.M. (1996). The Biosynthesis of Cellulose. J. Macromol. Sci. Part A 33: $1345-1373$.

Browse, J. (2009). Jasmonate Passes Muster: A Receptor and Targets for the Defense Hormone. Annu. Rev. Plant Biol. 60: 183-205.

Browse, J. and Howe, G.A. (2008). New Weapons and a Rapid Response against Insect Attack. Plant Physiol. 146: 832-838.

Bugbee, W.M. (1993). A pectin Lyase Inhibitor Protein from Cell Walls of Sugar Beet. Phytopathology 83: 63.

Burton, R.A., Gidley, M.J., and Fincher, G.B. (2010). Heterogeneity in the 
chemistry, structure and function of plant cell walls. Nat. Chem. Biol. 6: 724732.

Caffall, K.H. and Mohnen, D. (2009). The structure, function, and biosynthesis of plant cell wall pectic polysaccharides. Carbohydr. Res. 344: 1879-1900.

Capasso, J.M. and Hirschberg, C.B. (1984). Mechanisms of glycosylation and sulfation in the Golgi apparatus: evidence for nucleotide sugar/nucleoside monophosphate and nucleotide sulfate/nucleoside monophosphate antiports in the Golgi apparatus membrane. Proc. Natl. Acad. Sci. U. S. A. 81: 7051-5.

Cavalier, D.M., Lerouxel, O., Neumetzler, L., Yamauchi, K., Reinecke, A., Freshour, G., Zabotina, O.A., Hahn, M.G., Burgert, I., Pauly, M., Raikhel, N. V., and Keegstra, K. (2008). Disrupting Two Arabidopsis thaliana Xylosyltransferase Genes Results in Plants Deficient in Xyloglucan, a Major Primary Cell Wall Component. Plant Cell 20: 1519-1537.

Cervone, F., Hahn, M.G., De Lorenzo, G., Darvill, A., and Albersheim, P. (1989). Host-Pathogen Interactions : XXXIII. A Plant Protein Converts a Fungal Pathogenesis Factor into an Elicitor of Plant Defense Responses. Plant Physiol. 90: 542-8.

Cervone, F., De Lorenzo, G., Pressey, R., Darvill, A.G., and Albersheim, P. (1990). Can Phaseolus PGIP inhibit pectic enzymes from microbes and plants? Phytochemistry 29: 447-449.

Chaliha, C., Rugen, M.D., Field, R.A., and Kalita, E. (2018). Glycans as Modulators of Plant Defense Against Filamentous Pathogens. Front. Plant Sci. 9.

Chanliaud and Gidley (1999). In vitro synthesis and properties of pectin/Acetobacter xylinus cellulose composites. Plant J. 20: 25-35.

Chassot, C., Buchala, A., Schoonbeek, H.-J., Métraux, J.-P., and Lamotte, 0. (2008). Wounding of Arabidopsis leaves causes a powerful but transient protection against Botrytis infection. Plant J. 55: 555-67.

Clark, C.A. (1976). Comparative Histopathology of Botrytis squamosa and B. 
cinerea on Onion Leaves. Phytopathology 66: 1279.

Claverie, J., Balacey, S., Lemaître-Guillier, C., Brulé, D., Chiltz, A., Granet, L., Noirot, E., Daire, X., Darblade, B., Héloir, M.-C., and Poinssot, B. (2018).

The Cell Wall-Derived Xyloglucan Is a New DAMP Triggering Plant Immunity in Vitis vinifera and Arabidopsis thaliana. Front. Plant Sci. 9: 1725.

Coenen, G.J., Bakx, E.J., Verhoef, R.P., Schols, H.A., and Voragen, A.G.J. (2007). Identification of the connecting linkage between homo- or xylogalacturonan and rhamnogalacturonan type I. Carbohydr. Polym. 70: 224235.

Cosgrove, D.J. (2005). Growth of the plant cell wall. Nat. Rev. Mol. Cell Biol. 6: 850-61.

Côté, F. and Hahn, M.G. (1994b). Oligosaccharins: structures and signal transduction. Plant Mol. Biol. 26: 1379-411.

Creelman, R.A. and Mullet, J.E. (1997). BIOSYNTHESIS AND ACTION OF JASMONATES IN PLANTS. Annu. Rev. Plant Physiol. Plant Mol. Biol. 48: 355381.

Crombie, H.J. and Reid, J.S.G. (1998). Pectin methyltransferase: activities in particulate and solubilised preparations from mung bean (Vigna radiata) hypocotyls and tomato (Lycopersicon esculentum) pericarp. In 8th International Cell Walls Meeting.

D’Ovidio, R., Mattei, B., Roberti, S., and Bellincampi, D. (2004).

Polygalacturonases, polygalacturonase-inhibiting proteins and pectic oligomers in plant-pathogen interactions. Biochim. Biophys. Acta 1696: 237-44.

Darvill, A., Augur, C., Bergmann, C., Carlson, R.W., Cheong, J.J., Eberhard, S., Hahn, M.G., Ló, V.M., Marfa, V., Meyer, B. and Mohnen, D. (1992).

Oligosaccharins-oligosaccharides that regulate growth, development and defence responses in plants. Glycobiology 2: 181-198.

Darvill, J.E., McNeil, M., Darvill, A.G., and Albersheim, P. (1980). Structure of Plant Cell Walls: XI. GLUCURONOARABINOXYLAN, A SECOND 
HEMICELLULOSE IN THE PRIMARY CELL WALLS OF SUSPENSIONCULTURED SYCAMORE CELLS. Plant Physiol. 66: 1135-9.

Davis, K.R., Darvill, A.G., Albersheim, P., and Dell, A. (1986). Host-Pathogen Interactions : XXIX. Oligogalacturonides Released from Sodium Polypectate by Endopolygalacturonic Acid Lyase Are Elicitors of Phytoalexins in Soybean. Plant Physiol. 80: 568-577.

Davis, K.R. and Hahlbrock, K. (1987). Induction of defense responses in cultured parsley cells by plant cell wall fragments. Plant Physiol. 84: 1286-90.

Dean, G.H., Zheng, H., Tewari, J., Huang, J., Young, D.S., Hwang, Y.T., Western, T.L., Carpita, N.C., McCann, M.C., Mansfield, S.D., and Haughn, G.W. (2007). The Arabidopsis MUM2 gene encodes a beta-galactosidase required for the production of seed coat mucilage with correct hydration properties. Plant Cell 19: 4007-21.

DeBolt, S. and Estevez, J.M. (2012). Current Challenges in Plant Cell Walls: Editorial Overview. Front. Plant Sci. 3.

Decreux, A. and Messiaen, J. (2005). Wall-associated Kinase WAK1 Interacts with Cell Wall Pectins in a Calcium-induced Conformation. Plant Cell Physiol. 46: 268-278.

Denby, K.J., Kumar, P., and Kliebenstein, D.J. (2004). Identification of Botrytis cinerea susceptibility loci in Arabidopsis thaliana. Plant J. 38: 473-486.

Denoux, C., Galletti, R., Mammarella, N., Gopalan, S., Werck, D., De Lorenzo, G., Ferrari, S., Ausubel, F.M., and Dewdney, J. (2008). Activation of Defense Response Pathways by OGs and Flg22 Elicitors in Arabidopsis Seedlings. Mol. Plant 1: 423-445.

Domingo, C., Roberts, K., Stacey, N.J., Connerton, I., Ruíz-teran, F., and McCann, M.C. (2002). A pectate lyase from Zinnia elegans is auxin inducible. Plant J. 13: 17-28.

Dumville, J.C. and Fry, S.C. (2000). Uronic acid-containing oligosaccharins: Their biosynthesis, degradation and signalling roles in non-diseased plant tissues. 
Plant Physiol. Biochem. 38: 125-140.

Ebringerová, A. and Heinze, T. (2000). Xylan and xylan derivatives - biopolymers with valuable properties, 1 . Naturally occurring xylans structures, isolation procedures and properties. Macromol. Rapid Commun. 21: 542-556.

Ebringerová, A., Hromádková, Z., and Heinze, T. (2005). Hemicellulose. In Polysaccharides I (Springer-Verlag: Berlin/Heidelberg), pp. 1-67.

Egelund, J., Damager, I., Faber, K., Olsen, C.-E., Ulvskov, P., and Petersen, B.L. (2008). Functional characterisation of a putative rhamnogalacturonan II specific xylosyltransferase. FEBS Lett. 582: 3217-22.

Egelund, J., Petersen, B.L., Motawia, M.S., Damager, I., Faik, A., Olsen, C.E., Ishii, T., Clausen, H., Ulvskov, P., and Geshi, N. (2006). Arabidopsis thaliana RGXT1 and RGXT2 Encode Golgi-Localized (1,3)- -D-Xylosyltransferases Involved in the Synthesis of Pectic Rhamnogalacturonan-II. Plant Cell 18: 25932607.

Eklof, J.M. and Brumer, H. (2010). The XTH Gene Family: An Update on Enzyme Structure, Function, and Phylogeny in Xyloglucan Remodeling. Plant Physiol. 153: 456-466.

Ellis, C. (2002). The Arabidopsis Mutant cev1 Links Cell Wall Signaling to Jasmonate and Ethylene Responses. Plant Cell 14: 1557-1566.

Engelsdorf, T., Will, C., Hofmann, J., Schmitt, C., Merritt, B.B., Rieger, L., Frenger, M.S., Marschall, A., Franke, R.B., Pattathil, S., and Voll, L.M. (2017). Cell wall composition and penetration resistance against the fungal pathogen Colletotrichum higginsianum are affected by impaired starch turnover in Arabidopsis mutants. J. Exp. Bot. 68: 701-713.

Estornell, L.H., Wildhagen, M., Pérez-Amador, M.A., Talón, M., Tadeo, F.R., and Butenko, M.A. (2015). The IDA Peptide Controls Abscission in Arabidopsis and Citrus. Front. Plant Sci. 6.

Farmer, E.E., Moloshok, T.D., Saxton, M.J., and Ryan, C.A. (1991).

Oligosaccharide signaling in plants. Specificity of oligouronide-enhanced plasma 
membrane protein phosphorylation. J. Biol. Chem. 266: 3140-5.

Farmer, E.E., Weber, H., and Vollenweider, S. (1998). Fatty acid signaling in Arabidopsis. Planta 206: 167-74.

Ferrari, S. (2013). Oligogalacturonides: plant damage-associated molecular patterns and regulators of growth and development. Front. Plant Sci. 4.

Ferrari, S. (2003). Tandemly Duplicated Arabidopsis Genes That Encode Polygalacturonase-Inhibiting Proteins Are Regulated Coordinately by Different Signal Transduction Pathways in Response to Fungal Infection. Plant Cell 15: 93-106.

Ferrari, S., Galletti, R., Denoux, C., De Lorenzo, G., Ausubel, F.M., and Dewdney, J. (2007). Resistance to Botrytis cinerea induced in Arabidopsis by elicitors is independent of salicylic acid, ethylene, or jasmonate signaling but requires PHYTOALEXIN DEFICIENT3. Plant Physiol. 144: 367-79.

Ferrari, S., Sella, L., Janni, M., De Lorenzo, G., Favaron, F., and D'Ovidio, R. (2012). Transgenic expression of polygalacturonase-inhibiting proteins in Arabidopsis and wheat increases resistance to the flower pathogen Fusarium graminearum. Plant Biol. (Stuttg). 14 Suppl 1: 31-8.

Fry, S.C. (2004). Primary cell wall metabolism: tracking the careers of wall polymers in living plant cells. New Phytol. 161: 641-675.

Funakawa, H. and Miwa, K. (2015). Synthesis of borate cross-linked rhamnogalacturonan II. Front. Plant Sci. 6.

Galletti, R., Denoux, C., Gambetta, S., Dewdney, J., Ausubel, F.M., De Lorenzo, G., and Ferrari, S. (2008). The AtrbohD-mediated oxidative burst elicited by oligogalacturonides in Arabidopsis is dispensable for the activation of defense responses effective against Botrytis cinerea. Plant Physiol. 148: 1695-706.

Geng, X., Jin, L., Shimada, M., Kim, M.G., and Mackey, D. (2014). The phytotoxin coronatine is a multifunctional component of the virulence armament of Pseudomonas syringae. Planta 240: 1149-1165.

Geshi, N., Jørgensen, B., and Ulvskov, P. (2004). Subcellular localization and 
topology of beta(1-->4)galactosyltransferase that elongates beta(1-->4)galactan side chains in rhamnogalacturonan I in potato. Planta 218: 862-8.

Geshi, N., Pauly, M., and Ulvskov, P. (2002). Solubilization of galactosyltransferase that synthesizes 1,4-beta-galactan side chains in pectic rhamnogalacturonan I. Physiol. Plant. 114: 540-548.

Gomez, L.D., Steele-King, C.G., Jones, L., Foster, J.M., Vuttipongchaikij, S., and McQueen-Mason, S.J. (2009). Arabinan metabolism during seed development and germination in Arabidopsis. Mol. Plant 2: 966-76.

Goubet, F., Council, L.N., and Mohnen, D. (1998). Identification and Partial Characterization of the Pectin Methyltransferase "HomogalacturonanMethyltransferase" from Membranes of Tobacco Cell Suspensions. Plant Physiol. 116: 337-347.

Goubet, F. and Mohnen, D. (1999). Subcellular localization and topology of homogalacturonan methyltransferase in suspension-cultured Nicotiana tabacum cells. Planta 209: 112-7.

Goujon, T., Minic, Z., El Amrani, A., Lerouxel, O., Aletti, E., Lapierre, C., Joseleau, J.-P., and Jouanin, L. (2003). AtBXL1, a novel higher plant (Arabidopsis thaliana) putative beta-xylosidase gene, is involved in secondary cell wall metabolism and plant development. Plant J. 33: 677-90.

Guillermo Berumen-Varela, V.-A.O.-J. and Martín-Ernesto Tiznado-Hernández, R.F.-V. (2018). Rhamnogalacturonan lyase: a pectin modification enzyme of higher plants. Emirates J. Food Agric.

Hadfield, K.A. and Bennett, A.B. (1998). Polygalacturonases: many genes in search of a function. Plant Physiol. 117: 337-43.

Hanke, D.E. and Northcote, D.H. (1975). Molecular visualization of pectin and DNA by ruthenium red. Biopolymers 14: 1-17.

Harholt, J., Suttangkakul, A., and Vibe Scheller, H. (2010). Biosynthesis of Pectin. Plant Physiol. 153: 384-395.

Harholt, J., Jensen, J.K., Sørensen, S.O., Orfila, C., Pauly, M. and Scheller, H.V. 
(2005). ARABINAN DEFICIENT 1 Is a Putative Arabinosyltransferase Involved in Biosynthesis of Pectic Arabinan in Arabidopsis. Plant Physiol. 140: 49-58.

Haughn, G.W. and Western, T.L. (2012). Arabidopsis Seed Coat Mucilage is a Specialized Cell Wall that Can be Used as a Model for Genetic Analysis of Plant Cell Wall Structure and Function. Front. Plant Sci. 3: 64.

ten Have, A., Tenberge, K.B., Benen, J.A.E., Tudzynski, P., Visser, J., and van Kan, J.A.L. (2002). The Contribution of Cell Wall Degrading Enzymes to Pathogenesis of Fungal Plant Pathogens. In Agricultural Applications (Springer Berlin Heidelberg: Berlin, Heidelberg), pp. 341-358.

Hernández-Mata, G., Mellado-Rojas, M.E., Richards-Lewis, A., López-Bucio, J., Beltrán-Peña, E., and Soriano-Bello, E.L. (2010). Plant Immunity Induced by Oligogalacturonides Alters Root Growth in a Process Involving Flavonoid Accumulation in Arabidopsis thaliana. J. Plant Growth Regul. 29: 441-454.

Hocq, L., Pelloux, J., and Lefebvre, V. (2017). Connecting HomogalacturonanType Pectin Remodeling to Acid Growth. Trends Plant Sci. 22: 20-29.

Holland, N., Holland, D., Helentjaris, T., Dhugga, K.S., Xoconostle-Cazares, B., and Delmer, D.P. (2000). A comparative analysis of the plant cellulose synthase (CesA) gene family. Plant Physiol. 123: 1313-24.

Howe, G.A. and Jander, G. (2008). Plant Immunity to Insect Herbivores. Annu. Rev. Plant Biol. 59: 41-66.

Hu, R., Li, J., Wang, X., Zhao, X., Yang, X., Tang, Q., He, G., Zhou, G., and Kong, Y. (2016). Xylan synthesized by Irregular Xylem 14 ( IRX14) maintains the structure of seed coat mucilage in Arabidopsis. J. Exp. Bot. 67: 1243-1257.

Ischebeck, T., Stenzel, I., and Heilmann, I. (2008). Type B phosphatidylinositol-4phosphate 5-kinases mediate Arabidopsis and Nicotiana tabacum pollen tube growth by regulating apical pectin secretion. Plant Cell 20: 3312-30.

Ishii, T. and Matsunaga, T. (2001). Pectic polysaccharide rhamnogalacturonan II is covalently linked to homogalacturonan. Phytochemistry 57: 969-74.

Ishii, T., Matsunaga, T., Pellerin, P., O'Neill, M.A., Darvill, A., and Albersheim, P. 
(1999). The plant cell wall polysaccharide rhamnogalacturonan II self-assembles into a covalently cross-linked dimer. J. Biol. Chem. 274: 13098-104.

Ishikawa, M., Kuroyama, H., Takeuchi, Y., and Tsumuraya, Y. (2000).

Characterization of pectin methyltransferase from soybean hypocotyls. Planta 210: 782-91.

Janbon, G., Himmelreich, U., Moyrand, F., Improvisi, L., and Dromer, F. (2001).

Cas $1 p$ is a membrane protein necessary for the O-acetylation of the

Cryptococcus neoformans capsular polysaccharide. Mol. Microbiol. 42: 453-67.

Jensen, J.K., Sørensen, S.O., Harholt, J., Geshi, N., Sakuragi, Y., Møller, I., Zandleven, J., Bernal, A.J., Jensen, N.B., Sørensen, C. and Pauly, M.

(2008). Identification of a Xylogalacturonan Xylosyltransferase Involved in Pectin Biosynthesis in Arabidopsis. Plant Cell 20: 1289-1302.

Jones, L., Milne, J.L., Ashford, D., McCann, M.C., and McQueen-Mason, S.J. (2005). A conserved functional role of pectic polymers in stomatal guard cells from a range of plant species. Planta 221: 255-64.

Jones, L., Milne, J.L., Ashford, D., and McQueen-Mason, S.J. (2003). Cell wall arabinan is essential for guard cell function. Proc. Natl. Acad. Sci. 100: 1178311788.

Kachroo, A. (2003). Plastidial Fatty Acid Signaling Modulates Salicylic Acid- and Jasmonic Acid-Mediated Defense Pathways in the Arabidopsis ssi2 Mutant. Plant Cell 15: 2952-2965.

Kaida, R., Sugawara, S., Negoro, K., Maki, H., Hayashi, T., and Kaneko, T.S. (2010). Acceleration of cell growth by xyloglucan oligosaccharides in suspension-cultured tobacco cells. Mol. Plant 3: 549-54.

Kauss, H. and Swanson, A.L. (1969). Cooperation of Enzymes Responsible for Polymerisation and Methylation in Pectin Biosynthesis. Zeitschrift für Naturforsch. B 24: 28-33.

Keegstra, K. (2010). Plant Cell Walls. Plant Physiol. 154: 483-486.

Knox, J.P. (1992). Cell adhesion, cell separation and plant morphogenesis. Plant J. 


\section{2: $137-141$.}

Kohorn, B.D. and Kohorn, S.L. (2012). The cell wall-associated kinases, WAKs, as pectin receptors. Front. Plant Sci. 3: 88.

Kollárová, K., Henselová, M., and Lišková, D. (2005). Effect of auxins and plant oligosaccharides on root formation and elongation growth of mung bean hypocotyls. Plant Growth Regul. 46: 1-9.

Kollárová, K., Lišková, D., and Lux, A. (2007). Influence of galactoglucomannan oligosaccharides on root culture of Karwinskia humboldtiana. Plant Cell. Tissue Organ Cult. 91: 9-19.

Konishi, T., Takeda, T., Miyazaki, Y., Ohnishi-Kameyama, M., Hayashi, T., O'Neill, M.A., and Ishii, T. (2007). A plant mutase that interconverts UDParabinofuranose and UDP-arabinopyranose. Glycobiology 17: 345-54.

Konishi, T., Ono, H., Ohnishi-Kameyama, M., Kaneko, S. and Ishii, T. (2006). Identification of a Mung Bean Arabinofuranosyltransferase That Transfers Arabinofuranosyl Residues onto (1, 5)-Linked -L-Arabino-Oligosaccharides. Plant Physiol. 141: 1098-1105.

Körner, E., von Dahl, C.C., Bonaventure, G., and Baldwin, I.T. (2009). Pectin methylesterase NaPME1 contributes to the emission of methanol during insect herbivory and to the elicitation of defence responses in Nicotiana attenuata. J. Exp. Bot. 60: 2631-40.

Kravtchenko, T.P., Arnould, I., Voragen, A.G.J., and Pilnik, W. (1992). Improvement of the selective depolymerization of pectic substances by chemical $\beta$-elimination in aqueous solution. Carbohydr. Polym. 19: 237-242.

Kurata, Y., Mori, Y., Ishida, A., Nakajima, M., Ito, N., Hamada, M., Yamashita, K., Fujiwara, T., Tonosaki, M., and Katayama, Y. (2018). Variation in Hemicellulose Structure and Assembly in the Cell Wall Associated with the Transition from Earlywood to Latewood in Cryptomeria japonica. J. Wood Chem. Technol. 38: 254-263.

Laluk, K. and Mengiste, T. (2010). Necrotroph attacks on plants: wanton 
destruction or covert extortion? Arab. B. 8: e0136.

Larrieu, A. and Vernoux, T. (2016). Q\&amp;A: How does jasmonate signaling enable plants to adapt and survive? BMC Biol. 14: 79.

Lee, G.I. and Howe, G.A. (2003). The tomato mutant spr1 is defective in systemin perception and the production of a systemic wound signal for defense gene expression. Plant J. 33: 567-76.

Li, S., Wu, Q., Yin, F., Zhu, Z., He, J., and Barba, F. (2018). Development of a Combined Trifluoroacetic Acid Hydrolysis and HPLC-ELSD Method to Identify and Quantify Inulin Recovered from Jerusalem artichoke Assisted by Ultrasound Extraction. Appl. Sci. 8: 710.

Liners, F. and Van Cutsem, P. (1992). Distribution of pectic polysaccharides throughout walls of suspension-cultured carrot cells. Protoplasma 170: 10-21.

Liners, F., Letesson, J.-J., Didembourg, C., and Van Cutsem, P. (1989). Monoclonal Antibodies against Pectin: Recognition of a Conformation Induced by Calcium. Plant Physiol. 91: 1419-1424.

Lionetti, V., Cervone, F., and Bellincampi, D. (2012). Methyl esterification of pectin plays a role during plant-pathogen interactions and affects plant resistance to diseases. J. Plant Physiol. 169: 1623-30.

Lionetti, V., Fabri, E., De Caroli, M., Hansen, A.R., Willats, W.G.T., Piro, G., and Bellincampi, D. (2017). Three Pectin Methylesterase Inhibitors Protect Cell Wall Integrity for Arabidopsis Immunity to Botrytis. Plant Physiol. 173: 18441863.

Lionetti, V., Raiola, A., Cervone, F., and Bellincampi, D. (2014). Transgenic expression of pectin methylesterase inhibitors limits tobamovirus spread in tobacco and Arabidopsis. Mol. Plant Pathol. 15: 265-74.

Liu, N., Sun, Y., Pei, Y., Zhang, X., Wang, P., Li, X., Li, F., and Hou, Y. (2018). A Pectin Methylesterase Inhibitor Enhances Resistance to Verticillium Wilt. Plant Physiol. 176: 2202-2220.

De Lorenzo, G., Brutus, A., Savatin, D.V., Sicilia, F., and Cervone, F. (2011). 
Engineering plant resistance by constructing chimeric receptors that recognize damage-associated molecular patterns (DAMPs). FEBS Lett. 585: 1521-8.

De Lorenzo, G., Cervone, F., Bellincampi, D., Caprari, C., Clark, A.J., Desiderio, A., Devoto, A., Forrest, R., Leckie, F., and Nuss, L. (1994).

Polygalacturonase, PGIP and oligogalacturonides in cell-cell communication. Biochem. Soc. Trans. 22: 394-7.

De Lorenzo, G., D'Ovidio, R., and Cervone, F. (2001). The role of polygalacturonase-inhibiting proteins (PGIPs) in defense against pathogenic fungi. Annu. Rev. Phytopathol. 39: 313-35.

De Lorenzo, G. and Ferrari, S. (2002). Polygalacturonase-inhibiting proteins in defense against phytopathogenic fungi. Curr. Opin. Plant Biol. 5: 295-9.

Lorenzo, O. and Solano, R. (2005). Molecular players regulating the jasmonate signalling network. Curr. Opin. Plant Biol. 8: 532-540.

Lyon, G.D., Goodman, B.A., and Williamson, B. (2007). Botrytis cinerea Perturbs Redox Processes as an Attack Strategy in Plants. In Botrytis: Biology, Pathology and Control (Springer Netherlands: Dordrecht), pp. 119-141.

Maeda, K., Houjyou, Y., Komatsu, T., Hori, H., Kodaira, T., and Ishikawa, A. (2009). AGB1 and PMR5 contribute to PEN2-mediated preinvasion resistance to Magnaporthe oryzae in Arabidopsis thaliana. Mol. Plant. Microbe. Interact. 22: 1331-40.

von Malek, B., van der Graaff, E., Schneitz, K., and Keller, B. (2002). The Arabidopsis male-sterile mutant dde2-2 is defective in the ALLENE OXIDE SYNTHASE gene encoding one of the key enzymes of the jasmonic acid biosynthesis pathway. Planta 216: 187-92.

Manabe, Y., Nafisi, M., Verhertbruggen, Y., Orfila, C., Gille, S., Rautengarten, C., Cherk, C., Marcus, S.E., Somerville, S., Pauly, M. and Knox, J.P. (2011). Loss-of-function mutation of REDUCED WALL ACETYLATION2 in Arabidopsis leads to reduced cell wall acetylation and increased resistance to Botrytis cinerea. Plant Physiol. 155: 1068-78. 
Marin-Rodriguez, M.C. (2002). Pectate lyases, cell wall degradation and fruit softening. J. Exp. Bot. 53: 2115-2119.

Marowa, P., Ding, A., and Kong, Y. (2016). Expansins: roles in plant growth and potential applications in crop improvement. Plant Cell Rep. 35: 949-965.

Martín, I., Jiménez, T., Esteban, R., Dopico, B., and Labrador, E. (2008). Immunolocalization of a Cell Wall B-Galactosidase Reveals its Developmentally Regulated Expression in Cicer arietinum and its Relationship to Vascular Tissue. J. Plant Growth Regul. 27: 181-191.

Martín, I., Jiménez, T., Hernández-Nistal, J., Dopico, B., and Labrador, E. (2011). The $\beta \mathrm{I}$-galactosidase of Cicer arietinum is located in thickened cell walls such as those of collenchyma, sclerenchyma and vascular tissue. Plant Biol. 13: 777783.

Di Matteo, A., Bonivento, D., Tsernoglou, D., Federici, L., and Cervone, F. (2006). Polygalacturonase-inhibiting protein (PGIP) in plant defence: a structural view. Phytochemistry 67: 528-533.

Mauro, M.L., De Lorenzo, G., Costantino, P., and Bellincampi, D. (2002).

Oligogalacturonides inhibit the induction of late but not of early auxin-responsive genes in tobacco. Planta 215: 494-501.

McCann, M. and Carpita, N. (2008). Designing the deconstruction of plant cell walls. Curr. Opin. Plant Biol. 11: 314-320.

McNeil, M., Darvill, A.G., Fry, S.C., and Albersheim, P. (1984). Structure and Function of the Primary Cell Walls of Plants. Annu. Rev. Biochem. 53: 625-663.

Merced, A. and Renzaglia, K.S. (2019). Contrasting pectin polymers in guard cell walls of Arabidopsis and the hornwort Phaeoceros reflect physiological differences. Ann. Bot. 123: 579-585.

Mertz, R.A., Olek, A.T., and Carpita, N.C. (2012). Alterations in cell-wall glycosyl linkage structure of Arabidopsis murus mutants. Carbohydr. Polym. 89: 331339.

Micheli, F. (2001). Pectin methylesterases: cell wall enzymes with important roles in 
plant physiology. Trends Plant Sci. 6: 414-9.

Minic, Z., Rihouey, C., Do, C.T., Lerouge, P. and Jouanin, L. (2004). Purification and Characterization of Enzymes Exhibiting -D-Xylosidase Activities in Stem Tissues of Arabidopsis. Plant Physiol. 135: 867-878.

Moerschbacher, B.M., Mierau, Manfred;Graeßner, Barbara; Noll, U., and Mort, A.J. (1999). Small oligomers of galacturonic acid are endogenous suppressors of disease resistance reactions in wheat leaves. J. Exp. Bot. 50: 605-612.

Mohnen, D., Bar-Peled, M. and Somerville, C. (2008). Biosynthesis of Plant Cell Walls. In Biomass Recalcitrance, M. Himmel, ed (Blackwell Publishing, Oxford), pp. 94-187.

Mohnen, D. (2008). Pectin structure and biosynthesis. Curr. Opin. Plant Biol. 11: 266-77.

Moller, I., Sørensen, I., Bernal, A.J., Blaukopf, C., Lee, K., Øbro, J., Pettolino, F., Roberts, A., Mikkelsen, J.D., Knox, J.P., Bacic, A., and Willats, W.G.T. (2007). High-throughput mapping of cell-wall polymers within and between plants using novel microarrays. Plant J. 50: 1118-28.

Mouille, G., Ralet, M.-C., Cavelier, C., Eland, C., Effroy, D., Hématy, K., McCartney, L., Truong, H.N., Gaudon, V., Thibault, J.-F., Marchant, A., and Höfte, H. (2007). Homogalacturonan synthesis in Arabidopsis thaliana requires a Golgi-localized protein with a putative methyltransferase domain. Plant J. 50: 605-14.

Mutter, M., Renard, C.M.G.C., Beldman, G., Schols, H.A., and Voragen, A.G.J. (1998). Mode of action of RG-hydrolase and RG-lyase toward rhamnogalacturonan oligomers. Characterization of degradation products using RG-rhamnohydrolase and RG-galacturonohydrolase1Financed by Novo Nordisk A/S, Bagsvaerd, Denmark.1. Carbohydr. Res. 311: 155-164.

Nafisi, M., Stranne, M., Zhang, L., van Kan, J.A.L., and Sakuragi, Y. (2014). The Endo-Arabinanase BcAra1 Is a Novel Host-Specific Virulence Factor of the Necrotic Fungal Phytopathogen Botrytis cinerea. Mol. Plant-Microbe Interact. 27: 781-792. 
Nakano, T., Naito, Y., Kakegawa, K., Ohtsuki, N., Tsujimoto-Inui, Y., Shinshi, H., and Suzuki, K. (2012). Increase in pectin deposition by overexpression of an ERF gene in cultured cells of Arabidopsis thaliana. Biotechnol. Lett. 34: 763-9.

Neckelmann and Orellana (1998). Metabolism of uridine 5'-diphosphate-glucose in golgi vesicles from pea stems. Plant Physiol. 117: 1007-14.

Nishiyama, Y., Langan, P., and Chanzy, H. (2002). Crystal structure and hydrogenbonding system in cellulose Ibeta from synchrotron $X$-ray and neutron fiber diffraction. J. Am. Chem. Soc. 124: 9074-82.

Nishiyama, Y., Sugiyama, J., Chanzy, H., and Langan, P. (2003). Crystal structure and hydrogen bonding system in cellulose I(alpha) from synchrotron X-ray and neutron fiber diffraction. J. Am. Chem. Soc. 125: 14300-6.

Norman, C., Vidal, S., and Palva, E.T. (1999). Oligogalacturonide-Mediated Induction of a Gene Involved in Jasmonic Acid Synthesis in Response to the Cell-Wall-Degrading Enzymes of the Plant Pathogen Erwinia carotovora. Mol. Plant-Microbe Interact. 12: 640-644.

Novaković, L., Guo, T., Bacic, A., Sampathkumar, A., and Johnson, K. (2018). Hitting the Wall—Sensing and Signaling Pathways Involved in Plant Cell Wall Remodeling in Response to Abiotic Stress. Plants 7: 89.

Nunan, K.J. (2003). Solubilization of an Arabinan Arabinosyltransferase Activity from Mung Bean Hypocotyls. Plant Physiol. 132: 331-342.

Nuss, L., Mah é, A., Clark, A.J., Grisvard, J., Dron, M., Cervone, F., and De Lorenzo, G. (1996). Differential accumulation of PGIP (polygalacturonaseinhibiting protein) mRNA in two near-isogenic lines ofPhaseolus vulgarisL. upon infection withColletotrichum lindemuthianum. Physiol. Mol. Plant Pathol. 48: 8389.

O’Brien, J.A., Daudi, A., Finch, P., Butt, V.S., Whitelegge, J.P., Souda, P., Ausubel, F.M., and Bolwell, G.P. (2012). A peroxidase-dependent apoplastic oxidative burst in cultured Arabidopsis cells functions in MAMP-elicited defense. Plant Physiol. 158: 2013-27. 
O'Neill, M.A., Eberhard, S., Albersheim, P., and Darvill, A.G. (2001). Requirement of borate cross-linking of cell wall rhamnogalacturonan II for Arabidopsis growth. Science 294: 846-9.

O’Neill, M.A., Ishii, T., Albersheim, P., and Darvill, A.G. (2004).

Rhamnogalacturonan II: structure and function of a borate cross-linked cell wall pectic polysaccharide. Annu. Rev. Plant Biol. 55: 109-39.

O'Neill, M., Albersheim, P., and Darvill, A. (1990). The Pectic Polysaccharides of Primary Cell Walls. In Methods in plant biochemistry, pp. 415-441.

ØBro, J., Harholt, J., Scheller, H.V., and Orfila, C. (2004). Rhamnogalacturonan I in Solanum tuberosum tubers contains complex arabinogalactan structures. Phytochemistry 65: 1429-38.

Ochoa-Villarreal, M., Aispuro-Hernndez, E., Vargas-Arispuro, I., and Ngel, M. (2012). Plant Cell Wall Polymers: Function, Structure and Biological Activity of Their Derivatives. In Polymerization (InTech).

Orellana, A., Neckelmann, G., and Norambuena, L. (1997). Topography and Function of Golgi Uridine-5[prime]-Diphosphatase from Pea Stems. Plant Physiol. 114: 99-107.

Osorio, S., Castillejo, C., Quesada, M.A., Medina-Escobar, N., Brownsey, G.J., Suau, R., Heredia, A., Botella, M.A., and Valpuesta, V. (2008). Partial demethylation of oligogalacturonides by pectin methyl esterase 1 is required for eliciting defence responses in wild strawberry (Fragaria vesca). Plant J. 54: 4355.

Overmyer, K., Brosché, M., and Kangasjärvi, J. (2003). Reactive oxygen species and hormonal control of cell death. Trends Plant Sci. 8: 335-42.

Panayotatos, N. and Villemez, C.L. (1973). The formation of a -(1 leads to 4)-Dgalactan chain catalysed by a Phaseolus aureus enzyme. Biochem. J. 133: 263-71.

Pangesti, N., Reichelt, M., van de Mortel, J.E., Kapsomenou, E., Gershenzon, J., van Loon, J.J.A., Dicke, M., and Pineda, A. (2016). Jasmonic Acid and 
Ethylene Signaling Pathways Regulate Glucosinolate Levels in Plants During Rhizobacteria-Induced Systemic Resistance Against a Leaf-Chewing Herbivore. J. Chem. Ecol. 42: 1212-1225.

Passardi, F., Penel, C., and Dunand, C. (2004). Performing the paradoxical: how plant peroxidases modify the cell wall. Trends Plant Sci. 9: 534-540.

Pauly, M. and Scheller, H. V (2000). O-Acetylation of plant cell wall polysaccharides: identification and partial characterization of a rhamnogalacturonan O-acetyl-transferase from potato suspension-cultured cells. Planta 210: 659-67.

Pedras, M.S. and Khan, A.Q. (2000). Biotransformation of the phytoalexin camalexin by the phytopathogen Rhizoctonia solani. Phytochemistry 53: 59-69.

Pelloux, J., Rustérucci, C., and Mellerowicz, E.J. (2007). New insights into pectin methylesterase structure and function. Trends Plant Sci. 12: 267-77.

Petersen, B.L., Egelund, J., Damager, I., Faber, K., Jensen, J.K., Yang, Z., Bennett, E.P., Scheller, H.V., and Ulvskov, P. (2009). Assay and heterologous expression in Pichia pastoris of plant cell wall type-II membrane anchored glycosyltransferases. Glycoconj. J. 26: 1235-46.

Petrov, V., Hille, J., Mueller-Roeber, B., and Gechev, T.S. (2015). ROS-mediated abiotic stress-induced programmed cell death in plants. Front. Plant Sci. 6: 69.

Peugnet, I., Goubet, F., Bruyant-Vannier, M.-P., Thoiron, B., Morvan, C., Schols, H.A., and Voragen, A.G.J. (2001). Solubilization of rhamnogalacturonan I galactosyltransferases from membranes of a flax cell suspension. Planta 213: 435-445.

Philippe, F., Pelloux, J., and Rayon, C. (2017). Plant pectin acetylesterase structure and function: new insights from bioinformatic analysis. BMC Genomics 18: 456 .

Pieterse, C.M.J., Van der Does, D., Zamioudis, C., Leon-Reyes, A., and Van Wees, S.C.M. (2012). Hormonal Modulation of Plant Immunity. Annu. Rev. Cell Dev. Biol. 28: 489-521. 
Pogorelko, G., Lionetti, V., Fursova, O., Sundaram, R.M., Qi, M., Whitham, S.A., Bogdanove, A.J., Bellincampi, D., and Zabotina, O.A. (2013). Arabidopsis and Brachypodium distachyon transgenic plants expressing Aspergillus nidulans acetylesterases have decreased degree of polysaccharide acetylation and increased resistance to pathogens. Plant Physiol. 162: 9-23.

Popper, Z.A. (2008). Evolution and diversity of green plant cell walls. Curr. Opin. Plant Biol. 11: 286-92.

Popper, Z.A. and Fry, S.C. (2003). Primary cell wall composition of bryophytes and charophytes. Ann. Bot. 91: 1-12.

Rabinovich, G.A., van Kooyk, Y., and Cobb, B.A. (2012). Glycobiology of immune responses. Ann. N. Y. Acad. Sci. 1253: 1-15.

Rahman, T.A. El, Oirdi, M. El, Gonzalez-Lamothe, R., and Bouarab, K. (2012). Necrotrophic Pathogens Use the Salicylic Acid Signaling Pathway to Promote Disease Development in Tomato. Mol. Plant-Microbe Interact. 25: 1584-1593.

Raiola, A., Lionetti, V., Elmaghraby, I., Immerzeel, P., Mellerowicz, E.J., Salvi, G., Cervone, F., and Bellincampi, D. (2011). Pectin Methylesterase Is Induced in Arabidopsis upon Infection and Is Necessary for a Successful Colonization by Necrotrophic Pathogens. Mol. Plant-Microbe Interact. 24: 432-440.

Sundar Raj, A.A., Rubila, S., Jayabalan, R. and Ranganathan, T.V. (2012). A Review on Pectin: Chemistry due to General Properties of Pectin and its Pharmaceutical Uses. J. Cancer Sci. Ther. 1.

Rakitin, V.Y., Dolgikh, Y.I., Shaikina, E.Y., and Kuznetsov, V. V. (2001). Oligosaccharide Inhibits Ethylene Synthesis and Stimulates Somatic Embryogenesis in a Cotton Cell Culture. Russ. J. Plant Physiol. 48: 628-632.

Ralet, M.-C., Crépeau, M.-J., Vigouroux, J., Tran, J., Berger, A., Sallé, C., Granier, F., Botran, L., and North, H.M. (2016). Xylans Provide the Structural Driving Force for Mucilage Adhesion to the Arabidopsis Seed Coat. Plant Physiol. 171: 165-178.

Randoux, B., Renard-Merlier, D., Mulard, G., Rossard, S., Duyme, F., Sanssené, 
J., Courtois, J., Durand, R., and Reignault, P. (2010). Distinct defenses induced in wheat against powdery mildew by acetylated and nonacetylated oligogalacturonides. Phytopathology 100: 1352-63.

Ranf, S., Eschen-Lippold, L., Pecher, P., Lee, J., and Scheel, D. (2011). Interplay between calcium signalling and early signalling elements during defence responses to microbe- or damage-associated molecular patterns. Plant J. 68: 100-13.

Rasul, S., Dubreuil-Maurizi, C., Lamotte, O., Koen, E., Poinssot, B., Alcaraz, G., Wendehennie, D., and Jeandroz, S. (2012). Nitric oxide production mediates oligogalacturonide-triggered immunity and resistance to Botrytis cinerea in Arabidopsis thaliana. Plant. Cell Environ. 35: 1483-1499.

Reymond, P. and Farmer, E.E. (1998). Jasmonate and salicylate as global signals for defense gene expression. Curr. Opin. Plant Biol. 1: 404-11.

Reymond, P., Weber, H., Damond, M., and Farmer, E.E. (2000). Differential gene expression in response to mechanical wounding and insect feeding in Arabidopsis. Plant Cell 12: 707-20.

Richmond, T. (2000). Higher plant cellulose synthases. Genome Biol. 1: REVIEWS3001.

Ridley, B.L., O'Neill, M.A., and Mohnen, D. (2001). Pectins: structure, biosynthesis, and oligogalacturonide-related signaling. Phytochemistry 57: 92967.

Roberts, J. (2000). Cell Separation Processes in Plants-Models, Mechanisms and Manipulation. Ann. Bot. 86: 223-235.

Rogers, E.E., Glazebrook, J., and Ausubel, F.M. (1996). Mode of action of the Arabidopsis thaliana phytoalexin camalexin and its role in Arabidopsis-pathogen interactions. Mol. Plant. Microbe. Interact. 9: 748-57.

Rounds, C.M., Lubeck, E., Hepler, P.K., and Winship, L.J. (2011). Propidium lodide Competes with $\mathrm{Ca} 2+$ to Label Pectin in Pollen Tubes and Arabidopsis Root Hairs. Plant Physiol. 157: 175-187. 
Scheller, H.V., Doong, R. Lou, Ridley, B.L., and Mohnen, D. (1999). Pectin biosynthesis: a solubilized $\alpha 1,4$-galacturonosyltransferase from tobacco catalyzes the transfer of galacturonic acid from UDP-galacturonic acid onto the non-reducing end of homogalacturonan. Planta 207: 512-517.

Scheller, H.V. and Ulvskov, P. (2010). Hemicelluloses. Annu. Rev. Plant Biol. 61: 263-89.

Schmelz, E.A. (2003). Nitrogen Deficiency Increases Volicitin-Induced Volatile Emission, Jasmonic Acid Accumulation, and Ethylene Sensitivity in Maize. Plant Physiol. 133: 295-306.

Schouten, A., Tenberge, K.B., Vermeer, J., Stewart, J., Wagemakers, L., Williamson, B., and van Kan, J.A.L. (2002). Functional analysis of an extracellular catalase of Botrytis cinerea. Mol. Plant Pathol. 3: 227-238.

Schuhegger, R., Nafisi, M., Mansourova, M., Petersen, B.L., Olsen, C.E., Svatos, A., Halkier, B.A., and Glawischnig, E. (2006). CYP71B15 (PAD3) Catalyzes the Final Step in Camalexin Biosynthesis. Plant Physiol. 141: 1248-1254.

Seifert, G.J. and Blaukopf, C. (2010). Irritable walls: the plant extracellular matrix and signaling. Plant Physiol. 153: 467-78.

Selim, S., Sanssené, J., Rossard, S., and Courtois, J. (2017). Systemic Induction of the Defensin and Phytoalexin Pisatin Pathways in Pea (Pisum sativum) against Aphanomyces euteiches by Acetylated and Nonacetylated Oligogalacturonides. Molecules 22.

Sellam, A., Dongo, A., Guillemette, T., Hudhomme, P., and Simoneau, P. (2007). Transcriptional responses to exposure to the brassicaceous defence metabolites camalexin and allyl-isothiocyanate in the necrotrophic fungus Alternaria brassicicola. Mol. Plant Pathol. 8: 195-208.

Sham, A., Al-Azzawi, A., Al-Ameri, S., Al-Mahmoud, B., Awwad, F., AlRawashdeh, A., Iratni, R., and AbuQamar, S. (2014). Transcriptome analysis reveals genes commonly induced by Botrytis cinerea infection, cold, drought and oxidative stresses in Arabidopsis. PLoS One 9: e113718. 
Shlezinger, N., Minz, A., Gur, Y., Hatam, I., Dagdas, Y.F., Talbot, N.J., and Sharon, A. (2011). Anti-Apoptotic Machinery Protects the Necrotrophic Fungus Botrytis cinerea from Host-Induced Apoptotic-Like Cell Death during Plant Infection. PLoS Pathog. 7: e1002185.

Somerville, C. (2006). Cellulose synthesis in higher plants. Annu. Rev. Cell Dev. Biol. 22: 53-78.

Souza, C. de A., Li, S., Lin, A.Z., Boutrot, F., Grossmann, G., Zipfel, C., and Somerville, S.C. (2017). Cellulose-Derived Oligomers Act as DamageAssociated Molecular Patterns and Trigger Defense-Like Responses. Plant Physiol. 173: 2383-2398.

Spanu, P.D. and Panstruga, R. (2017). Editorial: Biotrophic Plant-Microbe Interactions. Front. Plant Sci. 8.

Sterling, J.D., Atmodjo, M.A., Inwood, S.E., Kumar Kolli, V.S., Quigley, H.F., Hahn, M.G., and Mohnen, D. (2006). Functional identification of an Arabidopsis pectin biosynthetic homogalacturonan galacturonosyltransferase. Proc. Natl. Acad. Sci. U. S. A. 103: 5236-41.

Sterling, J.D., Quigley, H.F., Orellana, A., and Mohnen, D. (2001). The catalytic site of the pectin biosynthetic enzyme alpha-1,4-galacturonosyltransferase is located in the lumen of the Golgi. Plant Physiol. 127: 360-71.

Sticklen, M.B. (2008). Plant genetic engineering for biofuel production: towards affordable cellulosic ethanol. Nat. Rev. Genet. 9: 433-43.

Stotz, H.U., Sawada, Y., Shimada, Y., Hirai, M.Y., Sasaki, E., Krischke, M., Brown, P.D., Saito, K., and Kamiya, Y. (2011). Role of camalexin, indole glucosinolates, and side chain modification of glucosinolate-derived isothiocyanates in defense of Arabidopsis against Sclerotinia sclerotiorum. Plant J. 67: 81-93.

Szklarczyk, D., Franceschini, A., Wyder, S., Forslund, K., Heller, D., HuertaCepas, J., Simonovic, M., Roth, A., Santos, A., Tsafou, K.P. and Kuhn, M. (2015). STRING v10: protein-protein interaction networks, integrated over the tree of life. Nucleic Acids Res. 43: D447-D452. 
Takeda, T., Furuta, Y., Awano, T., Mizuno, K., Mitsuishi, Y., and Hayashi, T. (2002). Suppression and acceleration of cell elongation by integration of xyloglucans in pea stem segments. Proc. Natl. Acad. Sci. 99: 9055-9060.

Tang, H., Belton, P.S., Ng, A., and Ryden, P. (1999). 13C MAS NMR studies of the effects of hydration on the cell walls of potatoes and Chinese water chestnuts. J. Agric. Food Chem. 47: 510-7.

Taurino, M., Abelenda, J.A., Río-Alvarez, I., Navarro, C., Vicedo, B., Farmaki, T., Jiménez, P., García-Agustín, P., López-Solanilla, E., Prat, S. and Rojo, E. (2014). Jasmonate-dependent modifications of the pectin matrix during potato development function as a defense mechanism targeted by Dickeya dadantii virulence factors. Plant J. 77: 418-429.

The United Nations 2012 Population Projections (2013). Popul. Dev. Rev. 39: 551-555.

Thomma, B.P.H.J., Eggermont, K., Penninckx, I.A.M.A., Mauch-Mani, B., Vogelsang, R., Cammue, B.P.A., and Broekaert, W.F. (1998). Separate jasmonate-dependent and salicylate-dependent defense-response pathways in Arabidopsis are essential for resistance to distinct microbial pathogens. Proc. Natl. Acad. Sci. 95: 15107-15111.

Tör, M., Lotze, M.T., and Holton, N. (2009). Receptor-mediated signalling in plants: molecular patterns and programmes. J. Exp. Bot. 60: 3645-54.

Toubart, P., Desiderio, A., Salvi, G., Cervone, F., Daroda, L., and De Lorenzo, G. (1992). Cloning and characterization of the gene encoding the endopolygalacturonase-inhibiting protein (PGIP) of Phaseolus vulgaris L. Plant J. 2: 367-73.

Tsai, A.Y.-L., Kunieda, T., Rogalski, J., Foster, L.J., Ellis, B.E., and Haughn, G.W. (2017). Identification and Characterization of Arabidopsis Seed Coat Mucilage Proteins. Plant Physiol. 173: 1059-1074.

Turner, J.G., Ellis, C., and Devoto, A. (2002). The Jasmonate Signal Pathway. Plant Cell 14: S153-S164. 
Uehara, Y., Tamura, S., Maki, Y., Yagyu, K., Mizoguchi, T., Tamiaki, H., Imai, T., Ishii, T., Ohashi, T., Fujiyama, K., and Ishimizu, T. (2017). Biochemical characterization of rhamnosyltransferase involved in biosynthesis of pectic rhamnogalacturonan I in plant cell wall. Biochem. Biophys. Res. Commun. 486: $130-136$.

Vannier, M.P., Thoiron, B., Morvan, C., and Demarty, M. (1992). Localization of methyltransferase activities throughout the endomembrane system of flax (Linum usitatissimum L) hypocotyls. Biochem. J. 286 ( Pt 3: 863-8.

Vargas-Rechia, Reicher, Rita Sierakowski M, Heyraud, Driguez, and Linart (1998). Xyloglucan octasaccharide XXLGol derived from the seeds of Hymenaea courbaril acts as a signaling molecule. Plant Physiol. 116: 1013-21.

Vega-Sanchez, M.E., Verhertbruggen, Y., Christensen, U., Chen, X., Sharma, V., Varanasi, P., Jobling, S.A., Talbot, M., White, R.G., Joo, M. and Singh, S. (2012). Loss of Cellulose Synthase-Like F6 Function Affects Mixed-Linkage Glucan Deposition, Cell Wall Mechanical Properties, and Defense Responses in Vegetative Tissues of Rice. Plant Physiol. 159: 56-69.

Veronese, P. (2006). The Membrane-Anchored BOTRYTIS-INDUCED KINASE1 Plays Distinct Roles in Arabidopsis Resistance to Necrotrophic and Biotrophic Pathogens. Plant Cell 18: 257-273.

Vijayan, P., Shockey, J., Levesque, C.A., Cook, R.J., and Browse, J. (1998). A role for jasmonate in pathogen defense of Arabidopsis. Proc. Natl. Acad. Sci. 95: 7209-7214.

Vincken, J.-P., Schols, H.A., Oomen, R.J.F.J., Beldman, G., Visser, R.G.F., and Voragen, A.G.J. (2003). Pectin - the Hairy Thing. In Advances in Pectin and Pectinase Research (Springer Netherlands: Dordrecht), pp. 47-59.

Vogel, J.P. (2002). PMR6, a Pectate Lyase-Like Gene Required for Powdery Mildew Susceptibility in Arabidopsis. Plant Cell 14: 2095-2106.

Vogel, J.P., Raab, T.K., Somerville, C.R., and Somerville, S.C. (2004). Mutations in PMR5 result in powdery mildew resistance and altered cell wall composition. Plant J. 40: 968-78. 
Voiniciuc, C., Dean, G.H., Griffiths, J.S., Kirchsteiger, K., Hwang, Y.T., Gillett, A., Dow, G., Western, T.L., Estelle, M., and Haughn, G.W. (2013). FLYING SAUCER1 Is a Transmembrane RING E3 Ubiquitin Ligase That Regulates the Degree of Pectin Methylesterification in Arabidopsis Seed Mucilage. Plant Cell 25: 944-959.

Voiniciuc, C., Engle, K.A., Günl, M., Dieluweit, S., Schmidt, M.H.-W., Yang, J.-Y., Moremen, K.W., Mohnen, D., and Usadel, B. (2018a). Identification of Key Enzymes for Pectin Synthesis in Seed Mucilage. Plant Physiol. 178: 1045-1064.

Voiniciuc, C., Guenl, M., Schmidt, M.H.-W., and Usadel, B. (2015). Highly Branched Xylan Made by IRX14 and MUCI21 Links Mucilage to Arabidopsis Seeds. Plant Physiol.: pp.01441.2015.

Voiniciuc, C., Pauly, M., and Usadel, B. (2018b). Monitoring Polysaccharide Dynamics in the Plant Cell Wall. Plant Physiol. 176: 2590-2600.

Voragen, A.G.J., Coenen, G.-J., Verhoef, R.P., and Schols, H.A. (2009). Pectin, a versatile polysaccharide present in plant cell walls. Struct. Chem. 20: 263-275.

de Vries, R.P., Kester, H.C., Poulsen, C.H., Benen, J.A., and Visser, J. (2000). Synergy between enzymes from Aspergillus involved in the degradation of plant cell wall polysaccharides. Carbohydr. Res. 327: 401-10.

Vriesmann, L.C., de Mello Castanho Amboni, R.D., and de Oliveira Petkowicz, C.L. (2011). Cacao pod husks (Theobroma cacao L.): Composition and hotwater-soluble pectins. Ind. Crops Prod. 34: 1173-1181.

Vurro, M., Bonciani, B., and Vannacci, G. (2010). Emerging infectious diseases of crop plants in developing countries: impact on agriculture and socio-economic consequences. Food Secur. 2: 113-132.

Walker, M., Tehseen, M., Doblin, M.S., Pettolino, F.A., Wilson, S.M., Bacic, A., and Golz, J.F. (2011). The Transcriptional Regulator LEUNIG_HOMOLOG Regulates Mucilage Release from the Arabidopsis Testa. Plant Physiol. 156: 46-60.

Wang, J., Dudareva, N., Bhakta, S., Raguso, R.A., and Pichersky, E. (1997). 
Floral scent production in Clarkia breweri (Onagraceae). II. Localization and developmental modulation of the enzyme S-adenosyl-L-methionine:(iso)eugenol O-methyltransferase and phenylpropanoid emission. Plant Physiol. 114: 21321.

Wang, X., Hou, S., Wu, Q., Lin, M., Acharya, B.R., Wu, D., and Zhang, W. (2017). IDL6-HAE/HSL2 impacts pectin degradation and resistance to Pseudomonas syringae pv tomato DC3000 in Arabidopsis leaves. Plant J. 89: 250-263.

Wasternack, C. and Song, S. (2016). Jasmonates: biosynthesis, metabolism, and signaling by proteins activating and repressing transciption. J. Exp. Bot: 443.

Watt, D.K., Ono, H., and Hayashi, K. (1998). Agrobacterium tumefaciens betaglucosidase is also an effective beta-xylosidase, and has a high transglycosylation activity in the presence of alcohols. Biochim. Biophys. Acta 1385: 78-88.

Weiberg, A., Wang, M., Lin, F.-M., Zhao, H., Zhang, Z., Kaloshian, I., Huang, H.D., and Jin, H. (2013). Fungal Small RNAs Suppress Plant Immunity by Hijacking Host RNA Interference Pathways. Science (80-. ). 342: 118-123.

Wen, F; Zhu, Y; Hawes, M. (1999). Effect of Pectin Methylesterase Gene Expression on Pea Root Development. Plant Cell 11: 1129-1140.

Western, T.L., Burn, J., Tan, W.L., Skinner, D.J., Martin-McCaffrey, L., Moffatt, B.A., and Haughn, G.W. (2001). Isolation and characterization of mutants defective in seed coat mucilage secretory cell development in Arabidopsis. Plant Physiol. 127: 998-1011.

Western, T.L., Skinner, D.J., and Haughn, G.W. (2000). Differentiation of Mucilage Secretory Cells of the Arabidopsis Seed Coat. Plant Physiol. 122: 345-356.

Whitehead, T.R. and Cotta, M.A. (2001). Identification of a broad-specificity xylosidase/arabinosidase important for xylooligosaccharide fermentation by the ruminal anaerobe Selenomonas ruminantium GA192. Curr. Microbiol. 43: 2938.

Wieczorek, K., Elashry, A., Quentin, M., Grundler, F.M.W., Favery, B., Seifert, 
G.J., and Bohlmann, H. (2014). A distinct role of pectate lyases in the formation of feeding structures induced by cyst and root-knot nematodes. Mol. Plant. Microbe. Interact. 27: 901-12.

Wilder, B.M. and Albersheim, P. (1973). The Structure of Plant Cell Walls: IV. A Structural Comparison of the Wall Hemicellulose of Cell Suspension Cultures of Sycamore (Acer pseudoplatanus) and of Red Kidney Bean (Phaseolus Vulgaris). Plant Physiol. 51: 889-93.

Willats, W.G.T., McCartney, L., Mackie, W., and Knox, J.P. (2001). Pectin: cell biology and prospects for functional analysis. Plant Mol. Biol. 47: 9-27.

Williamson, B., Tudzynski, B., Tudzynski, P., and Van Kan, J.A.L. (2007). Botrytis cinerea: the cause of grey mould disease. Mol. Plant Pathol. 8: 561580.

Wittstock, U. and Halkier, B.A. (2002). Glucosinolate research in the Arabidopsis era. Trends Plant Sci. 7: 263-70.

Yan, C. and Xie, D. (2015). Jasmonate in plant defence: sentinel or double agent? Plant Biotechnol. J. 13: 1233-1240.

Yao, C., Conway, W.S., Ren, R., Smith, D., Ross, G.S., and Sams, C.E. (1999). Gene encoding polygalacturonase inhibitor in apple fruit is developmentally regulated and activated by wounding and fungal infection. Plant Mol. Biol. 39: $1231-41$.

Yoda, H., Fujimura, K., Takahashi, H., Munemura, I., Uchimiya, H., and Sano, H. (2009). Polyamines as a common source of hydrogen peroxide in host- and nonhost hypersensitive response during pathogen infection. Plant Mol. Biol. 70: 103-12.

Yoshioka, H., Kashimura, Y., and Kaneko, K. (1995). beta;-D-Galactosidase and alpha;-L-Arabinofuranosidase Activities during the Softening of Apples. J. Japanese Soc. Hortic. Sci. 63: 871-878.

Zablackis, E., Huang, J., Muller, B., Darvill, A.G., and Albersheim, P. (1995). Characterization of the Cell-Wall Polysaccharides of Arabidopsis thaliana 
Leaves. Plant Physiol. 107: 1129-1138.

Zandleven, J., Beldman, G., Bosveld, M., Schols, H.A., and Voragen, A.G.J. (2006). Enzymatic degradation studies of xylogalacturonans from apple and potato, using xylogalacturonan hydrolase. Carbohydr. Polym. 65: 495-503.

\section{Zandleven, J., Sørensen, S.O., Harholt, J., Beldman, G., Schols, H.A., Scheller,} H. V., and Voragen, A.J. (2007). Xylogalacturonan exists in cell walls from various tissues of Arabidopsis thaliana. Phytochemistry 68: 1219-1226.

Zhang, H., Hong, Y., Huang, L., Li, D., and Song, F. (2016). Arabidopsis AtERF014 acts as a dual regulator that differentially modulates immunity against Pseudomonas syringae pv. tomato and Botrytis cinerea. Sci. Rep. 6: 30251.

Zhang, J., Shao, F., Li, Y., Cui, H., Chen, L., Li, H., Zou, Y., Long, C., Lan, L., Chai, J. and Chen, S. (2007). A Pseudomonas syringae effector inactivates MAPKs to suppress PAMP-induced immunity in plants. Cell Host Microbe 1: 175-85.

Zhang, L. and van Kan, J.A.L. (2013). Botrytis cinerea mutants deficient in d galacturonic acid catabolism have a perturbed virulence on Nicotiana benthamiana and Arabidopsis, but not on tomato. Mol. Plant Pathol. 14: 19-29.

Zhang, L., Thiewes, H., and van Kan, J.A.L. (2011). The d-galacturonic acid catabolic pathway in Botrytis cinerea. Fungal Genet. Biol. 48: 990-997.

Zhang, L., Zhang, F., Melotto, M., Yao, J., and He, S.Y. (2017). Jasmonate signaling and manipulation by pathogens and insects. J. Exp. Bot.: erw478.

Zhou, N. (1999). Arabidopsis PAD3, a Gene Required for Camalexin Biosynthesis, Encodes a Putative Cytochrome P450 Monooxygenase. Plant Cell 11: 2419_ 2428. 


\section{Acknowledgements}

This project is a culmination of tremendous amount of effort from many individuals. I am extremely grateful to my supervisor Dr. Till Ischebeck for his guidance during the more than 3 years he supervised me. I am grateful for the assistance he rendered, not only during lab hours but also outside the lab. His motivation kept me going even during the most frustrating of days in the lab. I am also indebted to members of my thesis committee, Prof. Dr. Volker Lipka and Prof. Dr. George Haughn for their ideas and guidance during this research endeavour. Many thanks to Prof George Haughn for also hosting me in his lab at the University of British Columbia in Canada. I would like to thank Prof. Dr. Petr Karlovsky, Prof. Dr. Kai Heimel, and Dr. Marcel Wiermer for being members of my examination board.

I am also grateful to Professor. Dr. Ivo Feussner for giving me the opportunity to work in his lab. I would also like to thank Dr Marcel Weimer and Professor. Dr. Xin Li (University of British Columbia) for allowing me to carry out my infection assays in their labs.

A big thank you goes to the International Research Training Group 2172 "PRoTECT: Plant Responses To Eliminate Critical Threats" for affording me this wonderful opportunity to work and interact with prominent scientists. I also want to thank the IRTG coordinators Judith Wassiltschenko and Stefanie König for all the work they do in the background that allowed smooth flow of my work. I would also like to that the Goettingen Graduate School for Neurosciences, Biophysics and Molecular Biosciences (GGNB) for all the courses they facilitated during the three years.

I am also grateful to Dr Ellen Hornung and Dr Martin Fulda for all the assistance they gave me in the lab, and helping me to settle in the lab and tolerating my everyday problems. Thank you Ellen for those hand knitted sockings for Luke, they really kept him warm. I am also grateful to Dr Krzysztof Zienkiewicz for the phytohormone measurements, together with Sabine Freitag for preparing the samples and Dr. Cornelia Herrfurth for help with the GC-MS. I am also indebted to Denise Harkten for assistance with my Botrytis infection assays. A big thank you goes to Susanne Mester for taking good care of my plants. I would also like to thank Dr Tegan Haslam, Dr Amelie Kelly, Dr Agnieszka Zienkiewicz, Dr Kirstin Feussner and Dr Gillian Dean for 
all the scientific advice given to me in the different labs. I would also like to thank all students that I worked with as assistants or lab rotation students: Max Fernkrorn, Maurice Hädrich, Patricia Scholz and Maxmilian Sievers.

I would also like to thank my fellow PhD students at both University of British Columbia and University of Goettingen: Franziska Kretzschmar, Elisa Krawczyk, Katharina Vollheyde, Katharina Vollheyde, Hanno Reseman, Dmitrij Rekther, Sven Haroth, Yitse Liu, Milena Lewandowska, Robert McGee and Krešimir Šola for all the good moments in the lab, plus the beers.

I big thank you goes to my wife Marjory and the two boys Liam and Luke, for all their moral support even though they were miles away-you guys are the best team ever. Thank you very much to my Dad and siblings, and more importantly, thank you to my late mum for whom I dedicate this thesis, you will always be my inspiration.

Thank you very much. 
